\title{
MOTIVIC MILNOR FIBER OF A QUASI-ORDINARY HYPERSURFACE
}

\author{
PEDRO D. GONZÁLEZ PÉREZ AND MANUEL GONZÁLEZ VILLA
}

\begin{abstract}
Let $f:\left(\mathbf{C}^{d+1}, 0\right) \rightarrow(\mathbf{C}, 0)$ be a germ of complex analytic function such that its zero level defines an irreducible germ of quasi-ordinary hypersurface $(S, 0) \subset\left(\mathbf{C}^{d+1}, 0\right)$. We describe the motivic Igusa zeta function, the motivic Milnor fibre and the Hodge-Steenbrink spectrum of $f$ at 0 in terms of topological invariants of $(S, 0) \subset\left(\mathbf{C}^{d+1}, 0\right)$.
\end{abstract}

\section{INTRODUCTION}

An equidimensional germ $(S, 0)$ of complex analytic variety of dimension $d$ is quasi-ordinary (q.o.) if there exists a finite map $\pi:(S, 0) \rightarrow\left(\mathbf{C}^{d}, 0\right)$ which is unramified outside a normal crossing divisor of $\left(\mathbf{C}^{d}, 0\right)$. Q.o. surface singularities originated in the classical Jung's method to parametrize and resolve surface singularities (see [22]) and are related to the classification of singularities by Zariski's dimensionality type (see [27). In addition to the applications to equisingularity problems, the class of q.o. singularities is also of interest to test and study various open questions and conjectures for singularities in general, particularly in the hypersurface case. In many cases the results use the fractional power series parametrizations of q.o. singularities. The parametrizations allow explicit computations combining analytic, topological and combinatorial arguments (see for instance [26, 15, 33, 28, 3]).

It is natural to investigate new invariants of singularities arising in the development of motivic integration, such as the motivic Igusa zeta functions for the class of q.o. singularities with the hope to extend the methods or results to wider classes (for instance using Jung's approach).

Denef and Loeser have introduced several (topological, naive motivic Igusa and motivic Igusa) zeta functions associated to a germ $f:\left(\mathbf{C}^{d+1}, x\right) \rightarrow(\mathbf{C}, 0)$ of complex analytic function. All these zeta functions are inspired by the Igusa zeta function in the $p$-adic setting and admit an expression in terms of an embedded resolution of singularities (or log-resolution) of $f$. The monodromy conjecture predicts a relation between the poles of these zeta functions and the eigenvalues of the local monodromy of $f$ at points of $f\left(x_{1}, \ldots, x_{d+1}\right)=0$ (see [5, 6, 8, ).

Let us consider, for instance, the motivic Igusa zeta function $Z(f, T)_{x}$ of $f$. It is known to be a rational function with respect to the indeterminate $T$ with coefficients in certain Grothendieck ring of varieties. Furthermore, it determines some classical invariants of the Milnor fiber of $f$ at $x$ (see 9, 6, 8, 21]). Its definition can be given in terms of certain jet spaces.

The set $\mathcal{L}_{n}\left(\mathbf{C}^{d+1}\right)_{x}\left(\right.$ resp. $\left.\mathcal{L}\left(\mathbf{C}^{d+1}\right)_{x}\right)$ of $n$-jets of arcs (resp. of arcs) in $\mathbf{C}^{d+1}$ consists of $\varphi \in\left(\mathbf{C}[t] /\left(t^{n+1}\right)^{d+1}\right.$ (resp. $\left.\varphi \in \mathbf{C}[[t]]\right)$ such that $\varphi(0)=x$. If $n \geq 1$ the set

$$
\mathcal{X}_{n, 1}:=\left\{\varphi \in \mathcal{L}_{n}\left(\mathbf{C}^{d+1}\right)_{x} \mid f \circ \varphi=t^{n} \bmod t^{n+1}\right\},
$$

enjoys a natural action of the group $\mu_{n}$ of $n$-th roots of unity. This set defines a class in the monodromic Grothendieck ring $K_{0}^{\hat{\mu}}\left(\operatorname{Var}_{\mathbf{C}}\right)$ (see its definition in Section 1). The motivic Igusa zeta function $Z(f, T)_{x}$ is the generating series

$$
Z(f, T)_{x}:=\sum_{n \geq 0}\left[\mathcal{X}_{n, 1}\right] \mathbf{L}^{-n(d+1)} T^{n},
$$

2000 Mathematics Subject Classification. 32S25, 32S45, 32S35, 14M25.

Key words and phrases. quasi-ordinary singularities, motivic Igusa zeta function, topological zeta function, Hodge-Steenbrink spectrum.

Both authors are supported by MCI-Spain grant MTM2010-21740-C02. 
where $\mathbf{L}$ denotes the class of the affine line $\mathbf{A}_{\mathbf{C}}^{1}$ with the trivial $\mu_{n}$-action. The motivic Milnor fiber of $f$ at $x$ is defined as a certain limit

$$
\mathcal{S}_{f, x}:=-\lim _{T \rightarrow \infty} Z(f, T)_{x} \in K_{0}^{\hat{\mu}}\left(\operatorname{Var}_{\mathbf{C}}\right)\left[\mathbf{L}^{-1}\right] .
$$

Denef and Loeser have proven that $\mathcal{S}_{f, x}$ and $F_{x}$ have the same Hodge characteristic. Hence $\mathcal{S}_{f, x}$ determines the Hodge-Steenbrink spectrum of $f$ at $x$ (see [6] and Section 1).

In this paper we give formulas for these invariants when $f=0$ defines an irreducible germ $(S, 0)$ of quasi-ordinary hypersurface singularity at $0 \in \mathbf{C}^{d+1}$. The germ $(S, 0)$ is parametrized by a q.o. branch, a fractional power series $\zeta\left(x_{1}^{1 / n}, \ldots, x_{d}^{1 / n}\right)$, for some integer $n$. The q.o. branch has a finite set of characteristic monomials, generalizing the notion of characteristic pairs of plane branches (see [1, 26). These monomials classify the embedded topology of the germ $(S, 0) \subset\left(\mathbf{C}^{d+1}, 0\right)$ (see [26, 15]). The semiroots of $f$ are auxiliary functions $f_{i}:\left(\mathbf{C}^{d+1}, 0\right) \rightarrow$ $(\mathbf{C}, 0), i=0, \ldots, g$ such that the hypersurface germ with equation $f_{i}=0$, is a q.o. hypersurface parametrized by a suitable truncation of $\zeta$ (see [18, 33]). Embedded resolutions of $(S, 0)$ are built in [17, as a composition of local toric modifications in terms of the characteristic monomials. The functions $x_{1}, \ldots, x_{d}, f_{0}, \ldots, f_{g-1}, f_{g}=f$ define suitable local coordinates at intermediate points in these resolutions. See Sections 2 and 4

We begin by studying the order of contact of an $\operatorname{arc} \varphi$ in $\mathbf{C}^{d+1}$ with $\varphi(0)=0$, with the $(d+g+1)$-tuple of functions $F:=\left(x_{1}, \ldots, x_{d}, f_{0}, \ldots, f_{g}\right)$, i.e., we study the values of

$$
\operatorname{ord}_{t}(F \circ \varphi):=\left(\operatorname{ord}_{t}\left(x_{1} \circ \varphi\right), \ldots, \operatorname{ord}_{t}\left(x_{d} \circ \varphi\right), \operatorname{ord}_{t}\left(f_{0} \circ \varphi\right), \ldots, \operatorname{ord}_{t}\left(f_{g} \circ \varphi\right)\right) \text {. }
$$

If the $\operatorname{arc} \varphi$ does not factor through the hypersurface $V$ of equation $x_{1} \ldots x_{d} f_{0} \ldots f_{g}=0$ then $\operatorname{ord}_{t}(F \circ \varphi)$ belongs to $\mathbf{Z}_{>0}^{d+g+1}$. We characterize these orders as certain integral points in the support of a fan $\Theta$, consisting of cones in $\mathbf{R}^{d+g+1}$ of dimension $\leq d+1$ (see Theorem 6.11). This fan is determined explicitely in terms of the characteristic monomials. It coincides essentially with the one given in 17 Section 5.4 in connection with the combinatorial description of the toric embedded resolution. The fan $\Theta$ can be seen as the tropical set associated to the embedding of $\left(\mathbf{C}^{d+1}, 0\right)$ in $\left(\mathbf{C}^{d+g+1}, 0\right)$ defined by this sequence of functions.

The second key step is to determine the motivic measure $\mu\left(H_{k, 1}\right)$ of the set

$$
H_{k, 1}:=\left\{\varphi \in \mathcal{L}\left(\mathbf{C}^{d+1}\right)_{0} \mid \operatorname{ord}_{t}(F \circ \varphi)=k, \quad f \circ \varphi=t^{k_{d+g+1}} \quad \bmod k_{d+g+1}\right\} .
$$

We reach this goal by applying the change of variables formula for motivic integrals to a toric embedded resolution of $(S, 0)$. Since $H_{k, 1}$ has a natural action of the group of $k_{d+g+1}$-th roots of unity, this measure is an element of the monodromic Grothendieck ring $K_{0}^{\hat{\mu}}\left(\operatorname{Var}_{\mathbf{C}}\right)\left[\mathbf{L}^{-1}\right]$.

Taking into account that the set $\mathcal{X}_{n, 1}$ is a disjoint union of sets of the form $H_{k, 1}$ we get that:

$$
Z(f, T)_{0}=\sum \mu\left(H_{k, 1}\right) T^{k_{d+g+1}}
$$

(see Proposition 8.1). The main result provides formulas for the motivic Igusa zeta function, the motivic naive Igusa zeta function, the topological zeta function and the motivic Milnor fiber of a q.o. germ $f:\left(\mathbf{C}^{d+1}, 0\right) \rightarrow(\mathbf{C}, 0)$ in terms of the characteristic monomials (see Theorem 3.7). As an application we obtain a formula for the Hodge-Steenbrink spectrum of $f$ at 0 (see Corollary 3.11) and also formulas for a finite set of candidate poles of the topological zeta function $Z(f, s)_{0}$ (see Corollary 3.17). We deduce that these invariants are determined by the embedded topological type of the q.o. hypersurface $(S, 0)$. Our results generalize those obtained when $d=1$ by Guibert [20, Schrauwen, Steenbrink and Stevens [38] Saito [36], and Artal et al. [3].

The naive motivic zeta function of a germ of complex analytic function defining a q.o. hypersurface, not necessarily irreducible, was studied by Artal Bartolo, Cassou-Nogués, Luengo and Melle-Hernández in [3] to prove the monodromy conjecture for this class of singularities. In Section 9 we compare the method of Newton maps used in [3] and the toric resolutions of [17, with the help of quotients of $\mathbf{C}^{d+1}$ by finite abelian groups. We point out in Example 10.1 the phenomenon of false reducibility, discovered in [19], which may appear when using Newton maps. This phenomenon, in the case $d \geq 2$, leads to inaccurate formulas for the zeta functions in [3. However, we check that the set of candidate poles which arise by our method coincides with the one given in 3 . See Remarks $3.10,3.18$ and 9.16 
An application to the description of the log-canonical threshold for this class of singularities will be given elsewhere. In the case $d=1$, see for instance [4.

Acknowledgments: We are grateful to Nero Budur, Alejandro Melle and to András Némethi for several discussions and their useful suggestions concerning this paper. The second author is also grateful to Enrique Artal-Bartolo, Mirel Căibar, Pierrette Cassou-Nogués, Gary Kennedy, Anatoly Libgober, Ignacio Luengo, Laurentiu Maxim, Lee McEwan and to the Alfred Renyi Institute of Mathematics (Hungary) and the Department of Mathematics, Statistics and Computer Sciences of the University of Illinois at Chicago (USA).

\section{INVARIANTS OF SINGULARITIES AND MOTIVIC INTEGRATION}

In this section we recall the definitions of the invariants we study in this paper.

The arc space of an algebraic variety. Let us denote by $\operatorname{Var}_{\mathbf{C}}$ the category of complex algebraic varieties, i.e., reduced and separated schemes of finite type over the complex field $\mathbf{C}$. If $0 \neq \varphi \in \mathbf{C}[[t]]$ we denote by $\operatorname{ord}_{t}(\varphi)$ (resp. $\operatorname{ac}(\varphi)$ the order (resp. angular coefficient) of the series $0 \neq \varphi \in \mathbf{C}[t]]$, that is, if $\varphi=t^{n} u$, where $u$ is a unit in $\mathbf{C}[[t]]$, then $\operatorname{ord}_{t}(\varphi)=n$ and $\operatorname{ac}(\varphi)=u(0) \in \mathbf{C}^{*}$.

If $n \geq 0$ the space $\mathcal{L}_{n}(X)$ of arcs modulo $t^{n+1}$ on a complex algebraic variety $X$ has also the structure of algebraic variety over $\mathbf{C}$. The canonical morphisms $\pi_{n}^{n^{\prime}}: \mathcal{L}_{n^{\prime}}(X) \rightarrow \mathcal{L}_{n}(X), n^{\prime}>n$ induced by truncation define a projective system. The arc space $\mathcal{L}(X)$ of $X$ is the projective limit of $\mathcal{L}_{n}(X)$. We consider the spaces $\mathcal{L}_{n}(X)$ and $\mathcal{L}(X)$ with their reduced structures. The arc space $\mathcal{L}(X)$ is a separated scheme over $\mathbf{C}$ but it is not of finite type in general. If $X$ is an affine variety given by the equations $f_{i}\left(x_{1}, \ldots, x_{d}\right)=0, i \in I$ then the $\mathbf{C}$-rational points of $\mathcal{L}_{n}(X)($ resp. of $\mathcal{L}(X))$ are $d$-tuplas $\varphi=\left(\varphi_{1}, \varphi_{2}, \ldots, \varphi_{d}\right) \in\left(\mathbf{C}[t] /\left(t^{n+1}\right)\right)^{d}\left(\right.$ resp. $\left.\left.\varphi \in(\mathbf{C}[[t]])^{d}\right)\right)$ such that $f_{i} \circ \varphi=0 \bmod t^{n+1},\left(\right.$ resp. $\left.f_{i} \circ \varphi=0\right)$, for all $i \in I$.

Truncating arcs mod $t^{n+1}$ defines a morphism of schemes $\pi_{n}: \mathcal{L}(X) \rightarrow \mathcal{L}_{n}(X)$. Note that $\pi_{0}(\mathcal{L}(X))=X$. For any $x \in X$ we denote $\left(\pi_{0}^{n}\right)^{-1}(x)$ by $\mathcal{L}_{n}(X)_{x}$ and $\left(\pi_{0}\right)^{-1}(x)$ by $\mathcal{L}(X)_{x}$. If the variety $X$ is smooth the maps $\pi_{n}$ are surjective and the maps $\pi_{n}^{n^{\prime}}$ are locally trivial fibrations with fibre $\mathbf{A}_{\mathbf{C}}^{\left(n^{\prime}-n\right) \operatorname{dim} X}$.

Grothendieck rings of algebraic varieties over C. The Grothendieck group of algebraic varieties over $\mathbf{C}$, is the abelian group $K_{0}\left(\operatorname{Var}_{\mathbf{C}}\right)$ generated by symbols $[X]$, where $X$ is a complex algebraic variety, with the relations $[X]=[Y]$ if $X$ and $Y$ are isomorphic, and $[X]=[Y]+[X \backslash Y]$ where $Y$ is a Zariski closed subset of $X$. Setting $[X]\left[X^{\prime}\right]:=\left[X \times_{\mathbf{C}} X^{\prime}\right]$ defines a ring structure on $K_{0}\left(\operatorname{Var}_{\mathbf{C}}\right)$ in such a way that 1 is equal to the class of a closed point. We denote by $\mathbf{L}$ the class of $\mathbf{A}_{\mathbf{C}}^{1}$ in $K_{0}\left(\operatorname{Var}_{\mathbf{C}}\right)$ and by $\mathcal{M}_{\mathbf{C}}$ the ring $K_{0}\left(\operatorname{Var}_{\mathbf{C}}\right)\left[\mathbf{L}^{-1}\right]$.

We denote by $\mu_{n}$ the algebraic group of $n$-roots of unity, i.e., $\mu_{n}=\operatorname{Spec}\left(\mathbf{C}[x] /\left(x^{n}-1\right)\right)$ for $n \geq 1$. The groups $\mu_{n}, n \geq 1$, together with the morphisms $\mu_{n d} \rightarrow \mu_{n}$ given by $x \mapsto x^{d}$ form a projective system. We denote by $\hat{\mu}$ its projective limit. A good $\mu_{n}$-action on a complex algebraic variety $X$ is a group action $\mu_{n} \times X \rightarrow X$ such that it is a morphism of algebraic varieties over $\mathbf{C}$ and each orbit is contained in an open affine subvariety of $X$. A good $\hat{\mu}$-action is a group action $\hat{\mu} \times X \rightarrow X$ which factors through a good $\mu_{n}$-action for some $n$.

The monodromic Grothendieck ring $K_{0}^{\hat{\mu}}\left(\operatorname{Var}_{\mathbf{C}}\right)$ of algebraic varieties over $\mathbf{C}$ is the abelian group on the symbols $[X, \hat{\mu}]$ where $X$ an algebraic variety with a good $\hat{\mu}$-action, with the relations $[X, \hat{\mu}]=[Y, \hat{\mu}]$ if $X$ and $Y$ are isomorphic as varieties with good $\hat{\mu}$-actions and $[X, \hat{\mu}]=$ $[Y, \hat{\mu}]+[X \backslash Y, \hat{\mu}]$, where $Y$ is a Zariski closed subset of $X$, considered with the $\hat{\mu}$-action induced by the one on $X$. We denote by $\mathbf{L}$ the affine line $\mathbf{A}_{\mathbf{C}}^{1}$ with the trivial $\hat{\mu}$-action. The product is defined as in $K_{0}\left(\operatorname{Var}_{\mathbf{C}}\right)$, by adding the relation $[X \times V, \hat{\mu}]=[X] \mathbf{L}^{d}$, for $V$ any $d$-dimensional affine space over $\mathbf{C}$ equipped by any linear $\hat{\mu}$-action. We denote by $\left[\mu_{n}\right] \in K_{0}^{\hat{\mu}}\left(\operatorname{Var}_{\mathbf{C}}\right)$ the class of $\mu_{n}$ equipped with the $\mu_{n}$ action; notice that $\left[\mu_{n}\right]=n \in K_{0}\left(\operatorname{Var}_{\mathbf{C}}\right)$ but $\left[\mu_{n}\right] \neq n \in K_{0}^{\hat{\mu}}\left(\operatorname{Var}_{\mathbf{C}}\right)$ if $n>1$. Another example is the following:

Example 1.1. Let $f \in \mathbf{C}\left[x_{1}^{ \pm 1}, \ldots, x_{m}^{ \pm 1}\right]$ be a quasi-homogeneous Laurent polynomial, i.e., there exists integers $D$ and $w_{1}, \ldots, w_{m}$ such that $f\left(t^{w_{1}} x_{1}, \ldots, t^{w_{m}} x_{m}\right)=t^{D} f\left(x_{1}, \ldots, x_{m}\right)$. Then $f=1$ 
defines a quasi-homogeneous hypersurface $V$ in the torus $\left(\mathbf{C}^{*}\right)^{m}$, which is endowed with the $\mu_{D^{-}}$ action given by $\alpha \cdot\left(x_{1}, \ldots, x_{m}\right) \mapsto\left(\alpha^{w_{1}} x_{1}, \ldots, \alpha^{w_{d}} x_{d}\right)$ for $\alpha \in \mu_{D}$.

Motivic integration. Let $X$ be a complex algebraic variety of pure dimension. A subset $A \subset \mathcal{L}(X)$ is cylinder if $A=\pi_{n}^{-1}(C)$ for some $n$ and some constructible subset $C \subset \mathcal{L}_{n}(X)$. A subset $A \subset \mathcal{L}(X)$ is stable if for some $m \in \mathbf{N}$ the set $\pi_{m}(A)$ is constructible, $A=\pi_{m}^{-1} \pi_{m}(A)$ and the morphism $\pi_{n+1}(A) \rightarrow \pi_{n}(A)$ is a piecewise locally trivial fibration with fibre $\mathbf{A}_{\mathbf{C}}^{\operatorname{dim} X}$, for $n>m$. If $X$ is smooth any cylinder is stable (see 7]).

We denote by $\hat{\mathcal{M}}_{\mathbf{C}}$ the completion of $\mathcal{M}_{\mathbf{C}}$ with respect to the filtration $F^{m} \mathcal{M}_{\mathbf{C}}$, with $m \in$ $\mathbf{Z}$, where $F^{m} \mathcal{M}_{\mathbf{C}}$ is the subgroup of $\mathcal{M}_{\mathbf{C}}$ generated by elements $[S] \mathbf{L}^{-i}$ with $S \in \operatorname{Var}_{\mathbf{C}}$ and $i-\operatorname{dim} S \geq m$.

Denef and Loeser have introduced a class of measurable subsets of $\mathcal{L}(X)$, containing the stable subsets, together with a measure $\mu_{X}(A) \in \hat{\mathcal{M}}_{\mathbf{C}}$, for any measurable set $A \subset \mathcal{L}(X)$, see [10, Appendix]. This motivic measure have the following properties:

\section{Proposition 1.2.}

(i) If $A \subset \mathcal{L}(X)$ is stable at level $n$, then $\mu_{X}(A)=\left[\pi_{n}(A)\right] \mathbf{L}^{-n \operatorname{dim} X}$.

(ii) If $A \subset \mathcal{L}(X)$ is contained in $\mathcal{L}(S)$ with $S$ a reduced closed subscheme of $X$ with $\operatorname{dim} S<$ $\operatorname{dim} X$, then $\mu_{X}(A)=0$.

(iii) Let $A_{i}$ with $i \in \mathbf{N}$ be a family of mutually disjoint measurable subsets of $\mathcal{L}(X)$. Assume that $A:=\cup_{i \in \mathbf{N}} A_{i}$ is a measurable set. Then $\sum_{i \in \mathbf{N}} \mu\left(A_{i}\right)$ converges to $\mu(A)$ in $\hat{\mathcal{M}}_{\mathbf{C}}$.

If $A \subset \mathcal{L}(X)$ is a measurable set, and $\alpha: A \rightarrow \mathbf{Z} \cup\{\infty\}$ is a function, then $\mathbf{L}^{-\alpha}$ is integrable on $A$ if the fibres of $\alpha$ are measurable, $\alpha^{-1}(\infty)$ has measure zero and the motivic integral $\int_{A} \mathbf{L}^{-\alpha} d \mu_{X}:=\sum_{n \in \mathbf{Z}} \mu_{X}\left(A \cap \alpha^{-1}(n)\right) \mathbf{L}^{-n}$ converges in $\hat{\mathcal{M}}_{\mathbf{C}}$.

Theorem 1.3. (Change variables formula [7, Lemma 3.3]) Let $\pi: Y \rightarrow X$ be a proper modification between two smooth complex algebraic varieties of pure dimension. Let $A$ be a measurable subset of $\mathcal{L}(X)$. Assume that $\pi$ induces a bijection between $B \subset \mathcal{L}(Y)$ and $A$. Then, for any function $\alpha: A \rightarrow \mathbf{Z} \cup\{\infty\}$ such that $\mathbf{L}^{-\alpha}$ is integrable on $A$, we have

$$
\int_{A} \mathbf{L}^{-\alpha} d \mu_{X}=\int_{B} \mathbf{L}^{-\alpha \circ \pi-\operatorname{ord}_{t} J_{\pi}(y)} d \mu_{Y}
$$

where $\operatorname{ord}_{t} J_{\pi}(y)$, for any $y \in \mathcal{L}(Y)$, denotes the order of the Jacobian of $\pi$ at $y$.

Remark 1.4. If the sets of arcs considered in Proposition [1.2 and Theorem [1.3 are equipped with a good $\hat{\mu}$-action then these results hold replacing $\mathcal{M}_{\mathbf{C}}\left(\operatorname{resp} . \hat{\mathcal{M}}_{\mathbf{C}}\right)$ by $\mathcal{M}_{\mathbf{C}}^{\hat{\mu}}\left(\operatorname{resp}\right.$. by $\left.\hat{\mathcal{M}}_{\mathbf{C}}^{\hat{\mu}}\right)$, where $\mathcal{M}_{\mathbf{C}}^{\hat{\mu}}:=K_{0}^{\hat{\mu}}\left(\operatorname{Var}_{\mathbf{C}}\right)\left[\mathbf{L}^{-1}\right]$ and $\hat{\mathcal{M}}_{\mathbf{C}}^{\hat{\mu}}$ is the completion of $\mathcal{M}_{\mathbf{C}}^{\hat{\mu}}$ with respect to the filtration introduced above.

Hodge characteristic and spectrum. A Hodge structure is a finite dimensional vector space over Q, equipped with a bigrading $H \otimes \mathbf{C}=\oplus_{p, q \in \mathbf{Z}} H^{p, q}$ such that $H^{p, q}$ is the complex conjugate of $H^{q, p}$ and each weight $m$ summand $\oplus_{p+q=m} H^{p, q}$ is defined over Q. Let HS (resp. HS ${ }^{\text {mon }}$ ) be the abelian category of Hodge structures (resp. equipped in addition with a quasi-unipotent endomorphism). The elements of the Grothendieck ring $K_{0}(\mathrm{HS})$ (resp. $K_{0}\left(\mathrm{HS}^{\mathrm{mon}}\right)$ ) are formal differences of isomorphic classes of Hodge structures (resp. equipped with a quasi-unipotent endomorphism). The product is induced by the tensor product of representatives.

A mixed Hodge structucture is a finite dimensional vector space $V$ with a finite increasing filtration $W_{\bullet} V$, such that the associated graded vector space $\mathrm{Gr}_{\bullet}^{W} V$ underlies a Hodge structure with $\mathrm{Gr}_{m}^{W} V$ as weight $m$ summand.

The canonical class $[V]:=\sum_{l}\left[\mathrm{Gr}_{l}^{W}\right] \in K_{0}(\mathrm{HS})$ is associated to the mixed Hodge structure $V$. If $X$ is a complex algebraic variety the simplicial cohomology groups $H_{c}^{i}(X, \mathbf{Q})$ of $X$, with compact support, have a mixed Hodge structure.

The Hodge characteristic of a complex algebraic variety $X$ is $\chi_{h}(X)=\sum_{i}(-1)^{i}\left[H_{c}^{i}(X, \mathbf{Q})\right] \in$ $K_{0}(\mathrm{HS})$. Since $\chi_{h}(\mathbf{L})$ is invertible in $K_{0}(\mathrm{HS})$ we get by additivity that $\chi_{h}$ extends to a ring homomorphism $\chi_{h}: \mathcal{M}_{\mathbf{C}} \rightarrow K_{0}$ (HS). If in addition $X$ is equipped with a good $\mu_{n}$-action then $\chi_{h}(X)$ can be seen as an element of $K_{0}\left(\mathrm{HS}^{\mathrm{mon}}\right)$ and we get in this way a ring homomorphism

$$
\chi_{h}^{\mathrm{mon}}: \mathcal{M}_{\mathbf{C}}^{\hat{\mu}} \rightarrow K_{0}\left(\mathrm{HS}^{\mathrm{mon}}\right),
$$


called the monodromic Hodge characteristic. We refer to 32, 34 for more on Hodge structures.

We denote by $\mathbf{Z}\left[t^{1 / \mathbf{Z}}\right]$ the ring $\bigcup_{n \geq 1} \mathbf{Z}\left[t^{1 / n}, t^{-1 / n}\right]$. The Hodge spectrum is a linear map

$$
h s p: K_{0}\left(\mathrm{HS}^{\mathrm{mon}}\right) \rightarrow \mathbf{Z}\left[t^{1 / \mathbf{Z}}\right]
$$

given by

$$
h s p([H])=\sum_{\alpha \in \mathbb{Q} \cap[0,1[} t^{\alpha}\left(\sum_{p, q \in \mathbf{Z}} h_{\alpha}^{p, q}(H) t^{p}\right),
$$

for $H$ a Hodge structure with a quasi-unipotent endomorphism, where $h_{\alpha}^{p, q}$ denotes the dimension of the eigenspace $H_{\alpha}^{p, q}$ corresponding to the eigenvector $e^{2 \pi i \alpha}$. The composition

$$
S p:=h s p \circ \chi_{h}^{\mathrm{mon}}: \mathcal{M}_{\mathbf{C}}^{\hat{\mu}} \longrightarrow \mathbf{Z}\left[t^{1 / \mathbf{Z}}\right]
$$

is a group homomorphism.

In the following Lemmas we recall some elementary properties of the map $S p$. See [4, 41 , for more properties and applications of this map.

Let $Q_{n, r}(t)$ denote the polynomial $\frac{t^{1 / n}-t}{1-t^{1 / n}} \frac{t^{1 / r}-t}{1-t^{1 / r}} \in \mathbf{Z}\left[t^{\frac{r}{n}}\right]$. We expand the polynomial $Q_{n, r}(t)=$ $\sum_{\alpha} c_{\alpha} t^{\alpha}$ and define the polynomials $Q_{n, r}^{<}(t):=\sum_{\alpha<1} c_{\alpha} t^{\alpha}$ and $Q_{n, r}^{>}(t):=\sum_{\alpha>1} c_{\alpha} t^{\alpha}$.

Lemma 1.5. We have the following properties:

(i) If $X$ is a complex algebraic variety, endowed with a good $\hat{\mu}$-action, then the equality $S p\left([X] \mathbf{L}^{d}\right)=S p([X]) t^{d}$ holds for any $d \in \mathbf{Z}_{\geq 0}$.

(ii) $\operatorname{Sp}\left(\left[\mu_{r}\right]\right)=\frac{1-t}{1-t^{1 / r}}$.

(iii) If $\operatorname{gcd}(n, r)=1$ then we have $\operatorname{Sp}\left(\left[\left\{(x, y) \in \mathbf{A}_{\mathbf{C}}^{2} \mid y^{n}-x^{r}=1\right\}\right]\right)=t-\frac{t^{1 / n}-t}{1-t^{1 / n}} \frac{t^{1 / r}-t}{1-t^{1 / r}}$.

(iv) If $\operatorname{gcd}(n, r)=1$ and $e>1$ the following equality holds

$$
S p\left(\left[\left\{(x, y) \in \mathbf{A}_{\mathbf{C}}^{2} \mid\left(y^{n}-x^{r}\right)^{e}=1\right\}\right]\right)=\frac{1-t}{1-t^{1 / e}}\left(t-Q_{n, r}^{<}\left(t^{1 / e}\right)-t^{\frac{e-1}{e}} Q_{n, r}^{>}\left(t^{1 / e}\right)\right) .
$$

Proof. Claim (i) can be seen as a consequence of (2.1.2) in [35. For (ii), (iii) and (iv) we refer to Lemma 3.4.2 and Lemma 3.4.3 of [20].

Notation 1.6. We denote the polynomial $S p\left(\left[\mu_{r}\right]\right)$ by $P_{r}$ (see Lemma 1.5). We set also $P_{n, r}:=S p\left(\left[\left\{(x, y) \in\left(\mathbf{C}^{*}\right)^{2} \mid y^{n}-x^{r}=1\right\}\right]\right)$ and $P_{n, r}^{(e)}:=S p\left(\left[\left\{(x, y) \in\left(\mathbf{C}^{*}\right)^{2} \mid\left(y^{n}-x^{r}\right)^{e}=1\right\}\right]\right)$.

Corollary 1.7. We have the following identities:

$$
\begin{aligned}
& P_{n, r}=t-\frac{t^{1 / n}-t}{1-t^{1 / n}} \frac{t^{1 / r}-t}{1-t^{1 / r}}-P_{n}-P_{r} \\
& P_{n, r}^{(e)}=P_{e}\left(t-Q_{n, r}^{<}\left(t^{1 / e}\right)-t^{\frac{e-1}{e}} Q_{n, r}^{>}\left(t^{1 / e}\right)\right)-P_{n e}-P_{r e}
\end{aligned}
$$

Proof. Let $n, r, e \in \mathbf{Z}_{>0}{ }^{d}$. The polynomial $f=y^{n}-x^{r}$ is quasi-homogeneuos with weights $w_{x}=n, w_{y}=r$ and degree $D=n r$. The identity

$$
\left[\left\{(x, y) \in \mathbf{C}^{2} \mid\left(y^{n}-x^{r}\right)^{e}=1\right\}\right]=\left[\mu_{n e}\right]+\left[\mu_{r e}\right]+\left[\left\{(x, y) \in\left(\mathbf{C}^{*}\right)^{2} \mid\left(y^{n}-x^{r}\right)^{e}=1\right\}\right]
$$

holds in $K_{0}^{\hat{\mu}}\left(\operatorname{Var}_{\mathbf{C}}\right)$ and follows from the partition of $\left\{(x, y) \in \mathbf{C}^{2} \mid\left(y^{n}-x^{r}\right)^{e}=1\right\}$ into three disjoint subsets: $\left\{(x, y) \in \mathbf{C}^{2} \mid y^{n e}=0, x^{r e}=1\right\},\left\{(x, y) \in \mathbf{C}^{2} \mid y^{n e}=1, x^{r e}=0\right\}$ and $\left\{(x, y) \in\left(\mathbf{C}^{*}\right)^{2} \mid\left(y^{n}-x^{r}\right)^{e}=1\right\}$. The $\mu_{D}$-action introduced in Example 1.1 is compatible with this partition. Then the identities hold by Lemma 1.5

The Milnor fiber and the Hodge-Steenbrink spectrum. Let $f: \mathbf{C}^{d+1} \rightarrow \mathbf{C}$ be a non constant morphism. Suppose that $f(x)=0$. Take $0<\delta<<\epsilon<1$ and denote by $B(x, \epsilon)$ the open ball of radius $\epsilon$ centered at $0 \in \mathbf{C}^{d+1}$, by $D_{\delta}$ the open disk of radius $\delta$ centered at $x \in \mathbf{C}$ and $D_{\delta}^{*}:=D_{\delta} \backslash\{0\}$. If $\epsilon$ and $\delta$ are small enough

$$
f: f^{-1}\left(D^{*}\right) \cap B(x, \epsilon) \rightarrow D_{\delta}^{*} .
$$

is a locally trivial fibration. The fibre $F_{x}:=f^{-1}(z) \cap B(x, \epsilon)$ for $z \in D_{\delta}^{*}$, which is defined up to isotopy, is called the Milnor fibre of $f$ at the point $x$. See [29].

The Milnor fibre is equipped with the monodromy operator $M_{f, x}: H^{\cdot}\left(F_{x}, \mathbf{Q}\right) \rightarrow H^{\cdot}\left(F_{x}, \mathbf{Q}\right)$ on the cohomology ring. By the Monodromy Theorem the operator $M_{f, x}$ is quasi-unipotent, that is, there exist $A, B \in \mathbf{Z}_{\geq 0}$ such that $\left(M_{f, x}^{A}-I\right)^{B}=0$ (see [25]). The cohomology groups 
$H^{i}\left(F_{x}, \mathbf{Q}\right)$ carry a natural mixed Hodge structure which is compatible with the monodromy operator $M_{f, x}$ (see [40] for isolated singularities and [30] for the general case). The Hodge characteristic of the Milnor fiber is defined as

$$
\chi_{h}^{\operatorname{mon}}\left(F_{x}\right)=\sum_{i}(-1)^{i}\left[H^{i}\left(F_{x}, \mathbf{Q}\right)\right] \in K_{0}\left(\mathrm{HS}^{\mathrm{mon}}\right) .
$$

Following [35, 6, 8, we introduce the Hodge-Steenbrink spectrum $S p^{\prime}(f, x)$ of $f$ at $x$ as:

Definition 1.8. We set

$$
S p^{\prime}(f, x):=(-1)^{d} h s p\left(\chi_{h}^{\operatorname{mon}}\left(F_{x}\right)-1\right) \quad \text { and } \quad S p(f, x):=t^{d+1} \iota\left(S p^{\prime}(f, x)\right),
$$

where $\iota: \mathbf{Z}\left[t^{1 / \mathbf{Z}}\right] \rightarrow \mathbf{Z}\left[t^{1 / \mathbf{Z}}\right]$ is the isomorphism such that $t^{\alpha} \mapsto t^{-\alpha}$.

Remark 1.9. There are several normalizations for the Hodge-Steenbrink spectrum in the literature. We have followed those of Saito [35]. In [6] $S p^{\prime}(f, x)$ (resp. $S p(f, x)$ ) is denoted by $H S p^{\prime}(f, x)$ (resp. $H S p(f, x)$ ), while in [8] and 20] the polynomial $S p^{\prime}(f, x)$ is denoted by $h \operatorname{sp}(f, x)$. In the paper [40] the Hodge-Steenbrink spectrum of $f$ at $x$ is defined to be the polynomial $S p(f, x)$, while in [41, 32, 42] the Hodge-Steenbrink spectrum of $f$ at $x$ differs of the polynomial $S p(f, x)$ by multiplication by $t$ (see 24). If $f$ has an isolated singular point at $x$ we have that $S p^{\prime}(f, x)=S p(f, x)$ (see [4]).

Motivic Igusa zeta function of a function at a point. Let $f: \mathbf{C}^{d+1} \rightarrow \mathbf{C}$ be a non constant morphism such that $f(x)=0$. For each natural $n$, we set

$$
\mathcal{X}_{n}:=\left\{\varphi \in \mathcal{L}_{n}\left(\mathbf{C}^{d+1}\right)_{x} \mid \operatorname{ord}_{t} f \circ \varphi=n\right\} .
$$

The set $\mathcal{X}_{n}$ is a locally closed subvariety of $\mathcal{L}_{n}\left(\mathbf{C}^{d+1}\right)_{x}$. We also consider the morphism

$$
\operatorname{ac}(f): \mathcal{X}_{n} \rightarrow \mathbf{C}^{*}, \varphi \mapsto \operatorname{ac}(f \circ \varphi) .
$$

There is a natural action of $\mathbf{C}^{*}$ on $\mathcal{X}_{n}$ given by $a \cdot \varphi(t)=\varphi(a t)$. Since $\operatorname{ac}(f)(a \cdot \varphi)=a^{n} \operatorname{ac}(f)(\varphi)$, the morphism $\operatorname{ac}(f)$ is a locally trivial fibration. We denote by $\mathcal{X}_{n, 1}$ the fibre $\operatorname{ac}(f)^{-1}(1)$. The previous $\mathbf{C}^{*}$-action on $\mathcal{X}_{n}$ restricts to a $\mu_{n}$-action on $\mathcal{X}_{n, 1}$.

Definition 1.10. 6]

(i) The naive motivic Igusa zeta function of $f$ at $x$ is

$$
Z^{\text {naive }}(f, T)_{x}:=\sum_{n \geq 1}\left[\mathcal{X}_{n}\right] \mathbf{L}^{-n(d+1)} T^{n} \in \hat{\mathcal{M}}_{\mathbf{C}}[[T]] .
$$

(ii) The motivic Igusa zeta function of $f$ at $x$ is

$$
Z(f, T)_{x}:=\sum_{n \geq 1}\left[\mathcal{X}_{n, 1}\right] \mathbf{L}^{-n(d+1)} T^{n} \in \hat{\mathcal{M}}_{\mathbf{C}}^{\hat{\mu}}[[T]] .
$$

If $A$ is $\mathcal{M}_{\mathbf{C}}$ or $\mathcal{M}_{\mathbf{C}}^{\hat{\mu}}$ we denote by $A[[T]]_{\text {sr }}$ the $A$-submodule of $A[[T]]$ generated by 1 and by finite products of terms $\mathbf{L}^{e} T^{i}\left(1-\mathbf{L}^{e} T^{i}\right)^{-1}$ with $e \in \mathbf{Z}$ and $i \in \mathbf{Z}_{>0}$.

Denef and Loeser gave formulas for $Z^{\text {naive }}(f, T)_{x}$ and $Z(f, T)_{x}$ in terms data associated to an embedded resolution of singularities of $f$ (see [9] Theorem 2.4, [6] Theorem 2.2.1). In particular they proved that

$$
Z^{\text {naive }}(f, T)_{x} \in \mathcal{M}_{\mathbf{C}}[[T]]_{\mathrm{sr}} \quad \text { and } \quad Z(f, T) \in \mathcal{M}_{\mathbf{C}}^{\hat{\mu}}[[T]]_{\mathrm{sr}} .
$$

The topological zeta function of $f$ at $x$ is a rational function $Z_{\text {top }}(f, s) \in \mathbf{Q}(s)$ defined by Denef and Loeser in [5] in terms of an embedded resolution of $f$. The motivic zeta function $Z^{\text {naive }}(f, T)_{x}$ determines $Z_{\text {top }}(f, s) \in \mathbf{Q}(s)$.

Proposition 1.11. 8, 6] We have that $Z_{\text {top }}(f, s)=\chi_{\text {top }}\left(Z^{\text {naive }}\left(f, \mathbf{L}^{-s}\right)\right)$, where $\chi_{\text {top }}$ denotes the topological Euler characteristic.

Lemma 1.12. [6, Lemma 4.1.1] There exists a unique ring homomorphism $\mathcal{M}_{\mathbf{C}}^{\hat{\mu}}[[T]]_{\mathrm{sr}} \rightarrow \mathcal{M}_{\mathbf{C}}^{\hat{\mu}}$, $\psi \mapsto \lim _{T \rightarrow \infty} \psi$, such that $\lim _{T \rightarrow \infty} \mathbf{L}^{e} T^{i}\left(1-\mathbf{L}^{e} T^{i}\right)^{-1}=-1$.

Lemma 1.13. If $\frac{p(T)}{q(T)} \in \mathcal{M}_{\mathbf{C}}^{\hat{\mu}}[[T]]_{\mathrm{sr}}$ and $\operatorname{deg}_{T} p(T)<\operatorname{deg}_{T} q(T)$ then we get $\lim _{T \rightarrow \infty} \frac{p(T)}{q(T)}=0$. 
Proof. It follows easily from Lemma 1.12

Definition 1.14. [9, 6] The motivic Milnor fiber of $f$ at the point $x$ is defined as

$$
\mathcal{S}_{f, x}:=-\lim _{T \rightarrow \infty} Z(f, T)_{x} \in \mathcal{M}_{\mathbf{C}}^{\hat{\mu}} .
$$

The motivic Milnor fiber $\mathcal{S}_{f, x}$ can be seen as a motivic incarnation of the classic Milnor fibre $F_{x}$ of $f$ at $x$. For instance $\mathcal{S}_{f, x}$ and $F_{x}$ have the same Hodge characteristic.

Theorem 1.15 (Denef and Loeser [9, 6]). The following equality holds in $K_{0}\left(\mathrm{HS}^{\mathrm{mon}}\right)$ :

$$
\chi_{h}^{\operatorname{mon}}\left(F_{x}\right)=\chi_{h}^{\operatorname{mon}}\left(\mathcal{S}_{f, x}\right) .
$$

Corollary 1.16. We have the equality $S p^{\prime}(f, x)=(-1)^{d} h s p\left(\chi_{h}\left(\mathcal{S}_{f, x}\right)-1\right)$.

Remark 1.17. The definitions and results of this section extend to the case when $f:\left(\mathbf{C}^{d+1}, x\right) \rightarrow$ $(\mathbf{C}, 0)$ is a germ of complex analytic function.

\section{QUASI-ORDINARY HYPERSURFACE SINGULARITIES}

A germ $(S, 0)$ of complex analytic variety equidimensional of dimension $d$ is quasi-ordinary (q.o.) if there exists a finite projection $\pi:(S, 0) \rightarrow\left(\mathbf{C}^{d}, 0\right)$ which is a local isomorphism outside a normal crossing divisor. If $(S, 0)$ is a hypersurface there is an embedding $(S, 0) \subset\left(\mathbf{C}^{d+1}, 0\right)$, defined by an equation $f=0$, where $f \in \mathbf{C}\left\{x_{1}, \ldots, x_{d}\right\}[y]$ is a q.o. polynomial, that is, a Weierstrass polynomial in $y$ with discriminant $\Delta_{y} f$ of the form $\Delta_{y} f=x^{\delta} u$ for a unit $u$ in the ring $\mathbf{C}\{x\}$ of convergent power series in the variables $x=\left(x_{1}, \ldots, x_{d}\right)$ and $\delta \in \mathbf{Z}_{\geq 0}^{d}$. In this coordinates the q.o. projection $\pi$ is the restriction of the projection

$$
\mathbf{C}_{\mathbf{C}}^{d+1} \rightarrow \mathbf{C}_{\mathbf{C}}^{d}, \quad\left(x_{1}, \ldots, x_{d}, y\right) \mapsto\left(x_{1}, \ldots, x_{d}\right) .
$$

We abuse of notation denoting the projection (11) also by $\pi$. The Jung-Abhyankar theorem guarantees that the roots of a q.o. polynomial $f$, called q.o. branches, are fractional power series in the ring $\mathbf{C}\left\{x^{1 / m}\right\}$, for some integer $m \geq 1$ (see [1]).

In this paper we suppose that the germ $(S, 0)$ is analytically irreducible, that is, the polynomial $f$ is irreducible in $\mathbf{C}\left\{x_{1}, \ldots, x_{d}\right\}[y]$.

Notation 2.1. If $\alpha, \beta \in \mathbf{Q}^{d}$ we consider the preorder relation given by $\alpha \leq \beta$ if $\beta \in \alpha+\mathbf{Q}_{\geq 0}^{d}$. We set also $\alpha<\beta$ if $\alpha \leq \beta$ and $\alpha \neq \beta$. The notation $\alpha \not \leq \beta$ means that the relation $\alpha \leq \beta$ does not hold. In $\mathbf{Q}^{d} \sqcup\{\infty\}$ we set that $\alpha<\infty$.

Lemma 2.2. [15, Proposition 1.3] Let $f \in \mathbf{C}\left\{x_{1}, \ldots, x_{d}\right\}[y]$ be an irreducible q.o. polynomial. Let $\zeta$ be a root of $f$ with expansion:

$$
\zeta=\sum c_{\lambda} x^{\lambda}
$$

There exist unique vectors $\lambda_{1}, \ldots, \lambda_{g} \in \mathbf{Q}_{>0}^{d}$ such that $\lambda_{1} \leq \cdots \leq \lambda_{g}$ and the three conditions below hold. We set the notations $\lambda_{0}=0, \lambda_{g+1}=\infty$, and introduce the lattices $M_{0}:=\mathbf{Z}^{d}$, $M_{j}:=M_{j-1}+\mathbf{Z} \lambda_{j}$, for $j=1, \ldots, g$.

(i) We have that $c_{\lambda_{j}} \neq 0$ for $j=1, \ldots, g$.

(ii) If $c_{\lambda} \neq 0$ then the vector $\lambda$ belongs to the lattice $M_{j}$, where $j$ is the unique integer such that $\lambda_{j} \leq \lambda$ and $\lambda_{j+1} \not \lambda$.

(iii) For $j=1, \ldots, g$, the vector $\lambda_{j}$ does not belong to $M_{j-1}$.

If $\zeta \in \mathbf{C}\left\{x^{1 / m}\right\}$ is a fractional power series satisfying the three conditions above then $\zeta$ is a q.o. branch.

Remark 2.3. Let us denote by $K$ the field of fractions of $\mathbf{C}\left\{x_{1}, \ldots, x_{d}\right\}$. If $\tau \in \mathbf{C}\left\{x_{1}^{1 / m}, \ldots, x_{d}^{1 / m}\right\}$ is a q.o. branch then the minimal polynomial $F \in K[y]$ of $\tau$ over $K$ has coefficients in the ring $\mathbf{C}\left\{x_{1}, \ldots, x_{d}\right\}$. Then the equation $F=0$ defines the q.o. hypersurface parametrized by $\tau$.

Definition 2.4. The exponents $\lambda_{1}, \ldots, \lambda_{g}$ in Lemma 2.2 (resp. the monomials $x^{\lambda_{1}}, \ldots, x^{\lambda_{g}}$ ) are called characteristic of the q.o. branch $\zeta$. The integers $n_{j}:=\left[M_{j-1}: M_{j}\right], j=1, \ldots, g$, are strictly greater than 0 by Lemma 2.2. We set also $n_{0}=1, e_{0}=n_{1} \cdots n_{g}$ and $e_{j}:=e_{0} /\left(n_{1} \ldots n_{j}\right)$ for $j=1, \ldots, g$. By convenience we denote by $M$ the lattice $M_{g}$ and by $N$ its dual lattice. 
Notation 2.5. We denote by $\left(\lambda_{j, 1}, \ldots, \lambda_{j, d}\right)$ the coordinates of the characteristic exponent $\lambda_{j}$ with respect to the canonical basis of $\mathbf{Q}^{d}$ and by $\geq_{\text {lex }}$ the lexicographic order.

Definition 2.6. The q.o. branch $\zeta$ is normalized if

$$
\left(\lambda_{1,1}, \ldots, \lambda_{g, 1}\right) \geq_{\text {lex }} \cdots \geq_{\operatorname{lex}}\left(\lambda_{1, d}, \ldots, \lambda_{g, d}\right),
$$

and if $\lambda_{1}$ is not of the form $\left(\lambda_{1,1}, 0, \ldots, 0\right)$ with $\lambda_{1,1}<1$.

Lipman proved that if the q.o. branch $\zeta$ is not normalized then there is a normalized q.o. branch $\zeta^{\prime}$ parametrizing the same germ and gave inversion formulas relating the characteristic monomials of $\zeta$ and $\zeta^{\prime}$ (see [15], Appendix). Lipman and Gau studied q.o. singularities from a topological point of view. They proved that the characteristic exponents of a normalized q.o. branch $\zeta$ parametrizing $(S, 0)$, classify the embedded topological type of the hypersurface germ $(S, 0) \subset\left(\mathbf{C}^{d+1}, 0\right)$ (see [15, 26]).

Lemma 2.7. If $\zeta$ is a q.o. branch of the form (2) then the series $\zeta_{j-1}:=\sum_{\lambda \ngtr \lambda_{j}} c_{\lambda} x^{\lambda}$ is a q.o. branch with characteristic exponents $\lambda_{1}, \ldots, \lambda_{j-1}$ for $j=0, \ldots, g$.

Proof. This is consequence of Lemma 2.2.

Definition 2.8. The q.o. branch $\zeta_{j}$ parametrizes the q.o. hypersurface $\left(S_{j}, 0\right)$. We denote by $f_{j} \in \mathbf{C}\left\{x_{1}, \ldots, x_{d}\right\}[y]$ the q.o. polynomial defining $\left(S_{j}, 0\right)$, for $j=0, \ldots, g-1$. By convenience we also denote $\zeta$ also by $\zeta_{g}$, hence $f=f_{g}$ and $S=S_{g}$.

Definition 2.9. The elements of $M$ defined by:

$$
\gamma_{1}=\lambda_{1} \quad \text { and } \quad \gamma_{j+1}-n_{j} \gamma_{j}=\lambda_{j+1}-\lambda_{j}, \quad \text { for } \quad j=1, \ldots, g-1 \text {, }
$$

together with the semigroup $\Gamma:=\mathbf{Z}_{\geq 0}^{d}+\gamma_{1} \mathbf{Z}_{\geq 0}+\cdots+\gamma_{g} \mathbf{Z}_{\geq 0} \subset \mathbf{Q}_{\geq 0}^{d}$ are associated with the q.o. polynomial $f$. We set $\gamma_{0}=0 \in \bar{M}$.

Lemma 2.10. [18, Proposition 3.1] We have that $f\left(\zeta_{j-1}\right)=x^{e_{0} \gamma_{j}} \cdot u_{j}$, where $u_{j}$ is a unit in $\mathbf{C}\left\{x^{1 / e_{0}}\right\}$, for $j=1, \ldots, g$.

Definition 2.11. [18, Definition 4.1] A q.o. hypersurface $\left(S^{\prime}, 0\right) \subset\left(\mathbf{C}^{d+1}, 0\right)$ is a $(j-1)$-th semi-root of $(S, 0)$ if it is parametrized by a q.o. branch $\tau$ with $j-1$ characteristic exponents and such that $f_{\mid y=\tau}=x^{e_{0} \gamma_{j}} \cdot u_{j}$, where $u_{j}$ is a unit in $\mathbf{C}\left\{x^{1 / e_{0}}\right\}$, for $j=1, \ldots, g$.

By Lemma 2.10 the germs $\left(S_{j}, 0\right)$, for $j=0, \ldots, g$ are semi-roots of $(S, 0)$. The semi-roots play an important role in the definition of the toric embedded resolution of the germ $(S, 0)$ below.

Lemma 2.12. The order of $\gamma_{j}+M_{j-1}$ in the finite abelian group $M_{j} / M_{j-1}$ is equal to $n_{j}$, for $j=1, \ldots, g$. We have the inequality

$$
n_{j} \gamma_{j}<\gamma_{j+1}, \text { for } j=1, \ldots, g \text {. }
$$

Definition 2.13. We denote by $r_{j}$ the integral length of the vector $n_{j} \gamma_{j}$ in the lattice $M_{j-1}$, for $j=1, \ldots, g$.

The integer $r_{j}$ is the greatest common divisor of the coordinates of the vector $n_{j} \gamma_{j}$ in terms of a basis of the lattice $M_{j-1}$.

Lemma 2.14. We have that $\operatorname{gcd}\left(r_{j}, n_{j}\right)=1$ for $j=1, \ldots, g$.

Proof. This follows from the first statement of Lemma 2.12 .

Lemma 2.15. Set $\lambda_{0}:=0 \in M$. We have the equality $\sum_{i=0}^{j} e_{i}\left(\lambda_{i+1}-\lambda_{i}\right)=e_{j} \gamma_{j+1}$, for $0 \leq j \leq g-1$.

Proof. We proceed by induction in $j$. For $j=0$ we have that $e_{0} \lambda_{1}=e_{0} \gamma_{1}$ because $\lambda_{1}=\gamma_{1}$. Suppose that it is true for $j$. We deduce the result from the equalities:

$$
\begin{gathered}
\sum_{i=1}^{j+1} e_{i}\left(\lambda_{i+1}-\lambda_{i}\right)=\sum_{i=1}^{j} e_{i}\left(\lambda_{i+1}-\lambda_{i}\right)+e_{j+1}\left(\lambda_{j+2}-\lambda_{j+1}\right)= \\
e_{j} \gamma_{j+1}+e_{j+1}\left(\lambda_{j+2}-\lambda_{j+1}\right)=e_{j+1}\left(n_{j+1} \gamma_{j+1}+\left(\lambda_{j+2}-\lambda_{j+1}\right)\right)=e_{j+1} \gamma_{j+2} .
\end{gathered}
$$


Notation 2.16. The canonical basis $\varepsilon_{1}, \ldots, \varepsilon_{d}$ of $\mathbf{Q}^{d}$ is also a basis of the lattice $M_{0}$ and a minimal set of generators of the semigroup $\mathbf{Z}_{>0}^{d} \subset M_{0}$. The dual basis $\epsilon_{1}, \ldots, \epsilon_{d}$ of the dual lattice $N_{0}$ spans a regular cone $\rho$ in $N_{0, \mathbf{R}}$. It follows that $\mathbf{R}_{>0}^{d}=\rho^{\vee}$ and $\mathbf{Z}_{>0}^{d}=\rho^{\vee} \cap M_{0}$, where $\rho^{\vee}$ denotes the dual cone of $\rho$ (see Notations of Section 4 ). We denote by $\langle\rangle:, N_{\mathbf{R}} \times M_{\mathbf{R}} \rightarrow \mathbf{R}$ the duality pairing associated to the dual vector spaces $N_{\mathbf{R}}$ and $M_{\mathbf{R}}$.

Notation 2.17. If $1 \leq j \leq g$ we denote by $\ell_{j}$ the number of nonzero coordinates of the characteristic exponent $\lambda_{j}$, with respect to the canonical basis $\varepsilon_{1}, \ldots, \varepsilon_{d}$ of $\mathbf{Q}^{d}$. We set $\ell_{0}:=0$.

Remark 2.18. We have that $0=\ell_{0}<\ell_{1} \leq \ell_{2} \leq \cdots \leq \ell_{g}$.

Notation 2.19. We denote by $\epsilon_{1}, \ldots, \epsilon_{d+g+1}$ the canonical basis of the lattice $\mathbf{Z}^{d+g+1}$. We consider the cone $\rho$ embedded as $\rho_{0}:=\rho \times\{0\} \subset \mathbf{R}^{d} \times \mathbf{R}^{g+1}$ (see Notation 2.16). The following cones in $\mathbf{R}^{d+g+1}$ are $d$-dimensional and simplicial

$$
\rho_{j}:=\left\{a+\sum_{i=1}^{j}\left\langle a, \gamma_{i}\right\rangle \epsilon_{d+i}+\sum_{i=j+1}^{g+1} n_{j} \cdots n_{i-1}\left\langle a, \gamma_{j}\right\rangle \epsilon_{d+i} \mid a \in \rho\right\}, \text { for } j=1, \ldots, g .
$$

We denote by $\nu_{i}^{(j)}$ the primitive integral vector in the edge of the cone $\rho_{j}$ defined by the vector $\epsilon_{i}+\sum_{r=1}^{j}\left\langle\epsilon_{i}, \gamma_{r}\right\rangle \epsilon_{d+r}+\sum_{r=j+1}^{g+1} n_{j} \cdots n_{r-1}\left\langle\epsilon_{i}, \gamma_{j}\right\rangle \epsilon_{d+r} \in \mathbf{Q}^{d+g+1}$. We introduce also the following cones of dimension $d+1$

$\sigma_{j}^{+}:=\rho_{j}+\mathbf{R}_{\geq 0} \epsilon_{d+j}, \quad \sigma_{j}^{-}:=\rho_{j-1}+\rho_{j}, \quad$ for $j=1, \ldots, g, \quad$ and $\quad \sigma_{g+1}:=\rho_{g}+\mathbf{R}_{\geq 0} \epsilon_{d+g+1}$.

We introduce a fan $\Theta$ in $\mathbf{R}^{d+g+1}$ associated to the q.o. polynomial $f$. This fan appears in [17. Section 5.4 ], in connexion with the definition of a toric embedded resolution of the q.o. hypersurface $(S, 0) \subset\left(\mathbf{C}^{d+1}\right)$.

Definition 2.20. We set $\Theta_{j}:=\left\{\sigma_{j}^{+}, \sigma_{j}^{-}, \rho_{j}\right\}$ for $j=1, \ldots, g$ and $\Theta_{g+1}:=\left\{\sigma_{g+1}\right\}$. The set $\Theta$ consisting of the faces of the cones in $\cup_{j=1}^{g+1} \Theta_{j}$, is a fan associated to the q.o. polynomial $f$.

Definition 2.21. The following functions are linear forms on $\mathbf{Z}^{d+g+1}$

$\eta(k)=k_{d+g+1}, \xi_{1}(k):=\sum_{i=1}^{d+1} k_{i}$, and $\xi_{j}(k):=\sum_{i=1}^{d} k_{i}+\sum_{i=1}^{j-1}\left(1-n_{i}\right) k_{d+i}+k_{d+j}$, for $j=2, \ldots, g+1$.

Lemma 2.22. If $k=\sum_{i=1}^{d+g+1} k_{i} \epsilon_{i} \in \mathbf{Z}^{d+g+1}$ is a primitive integral vector generating an edge of some cone $\theta \in \Theta_{j}$ for some $1 \leq j \leq g+1$ then $\xi_{j}(k) \geq 0$. If in addition $k \in \rho_{j-1}$ then we get $\xi_{j}(k)=\xi_{j-1}(k)$. If $k_{d+g+1}=0$ then we have that $\xi_{j}(k)=1$, morever in this case either $k=\epsilon_{d+j+1}$ or $k=\epsilon_{i}$ for some $1 \leq i \leq d$ such that the $i$-th coordinate of the characteristic exponent $\lambda_{j}$ is equal to zero.

Proof. Let us check that $\xi_{j}(k) \geq 0$. The claim is obvious for $j=1$ because the definition of $\xi_{1}(k)$. If $j>1$ and $k \in \rho_{j}$ then we get that $k_{d+i+1} \geq n_{i} k_{d+i}$ for $1 \leq i \leq j-1$ and $k_{d+i+1}=n_{i} k_{d+i}$ for $j \leq i \leq g$ because of the definition of $\rho_{j}$ and Lemma 2.12. In this case we get that $\xi_{j}(k)=\sum_{i=1}^{d+1} k_{i}+\sum_{i=1}^{j}\left(k_{d+i+1}-n_{i} k_{d+i}\right) \geq 0$. If $k \in \rho_{j-1}$ we have that $k_{d+j+1}=n_{j} k_{d+j}$ hence $\xi_{j}(k)=\sum_{i=1}^{d+1} k_{i}+\sum_{i=1}^{j-1}\left(k_{d+i+1}-n_{i} k_{d+i}\right)=\xi_{j-1}(k) \geq 0$. Finally, if $k=\epsilon_{d+j+1}$ and $1 \leq j \leq g$ we get that $\xi_{j}\left(\epsilon_{d+j+1}\right)=1$.

If $k \neq \epsilon_{d+j+1}, k_{d+g+1}=0$ and if $\nu:=\left(k_{1}, \ldots, k_{d}\right)$ we get that $\left\langle\nu, \gamma_{j}\right\rangle=0$ (see Notation 2.19), that is, the vector $\nu \in N_{0}$ is orthogonal to $\gamma_{j}$. Since $\nu$ defines an edge of $\rho$ it follows that $k=\epsilon_{i}$ for some $1 \leq i \leq d$ such that the i-th coordinate of $\gamma_{j}$ is equal to zero. Then the conclusion follows by relations (3).

\section{Statement of Main Results}

In this section we introduce the main results of the paper.

We recall first some basic facts on generating functions. The generating function of a set $A \subset \mathbf{Z}^{m}$ is the formal power series $\sum_{u \in A} x^{u}$.

Lemma 3.1. 39, Chapter 4, Theorem 4.6.11] If $\theta \in \mathbf{R}_{\geq 0}^{m}$ is a rational polyhedral cone for the lattice $\mathbf{Z}^{m}$ the generating function $G_{\theta}$ of the set $\operatorname{int}(\theta) \cap \mathbf{Z}^{m}$ is a rational function of the form 
$G_{\theta}=P_{\theta} \prod_{v}\left(1-x^{v}\right)^{-1}$, where $v$ runs through the set of primitive integral vectors in the edges of the cone $\theta$ and $P_{\theta}$ is a polynomial with integral coefficients and exponents in $\theta \cap \mathbf{Z}^{m}$. If in addition the cone $\theta$ is simplicial, we denote by $v_{1}, \ldots, v_{r} \in \mathbf{Z}^{m}$ the primitive integral vectors in the edges of $\theta$ and by $D_{\theta}$ the set $D_{\theta}:=\left\{\nu=\sum_{i=1}^{r} a_{i} v_{i} \in \operatorname{int}(\theta) \cap \mathbf{Z}^{m} \mid 0<a_{i} \leq 1\right\}$; then we get that $P_{\theta}=\sum_{\nu \in D_{\theta}} x^{\nu}$.

Lemma 3.2. With the hypothesis and notations of Lemma 3.1 we assume in addition that the cone $\theta$ is simplicial of dimension $r$. We consider two $\mathbf{Z}$-linear maps $\kappa, \iota: \mathbf{Z}^{m} \rightarrow \mathbf{Z}$ such that for any primitive integral vector in an edge of the cone $\theta$ we have $\iota(v) \geq 0$ and if $\iota(v)=0$ then $\kappa(v)=1$. We denote by $\tilde{G}_{\theta}$ the image of $G_{\theta}$ by the monomial map $x^{\nu} \mapsto \mathbf{L}^{-\kappa(\nu)} T^{\iota(\nu)}$ and by $r_{0}$ the number of rays $\mathbf{R}_{\geq 0} v$ of $\theta$ such that $\iota(v)=0$. We have that $\lim _{T \rightarrow \infty} \tilde{G}_{\theta}=$ $(-1)^{r-r_{0}}(\mathbf{L}-1)^{-r_{0}}$.

Proof. By Lemma 3.1 we get that $\tilde{G}_{\sigma}=\left(\sum_{\nu \in D_{\theta}} \mathbf{L}^{-\kappa(\nu)} T^{\iota(\nu)}\right) \prod_{i=1}^{r}\left(1-\mathbf{L}^{-\kappa\left(v_{i}\right)} T^{\iota\left(v_{i}\right)}\right)^{-1}$. The vector $\nu_{0}:=\sum_{i} v_{i}$ belongs to $D_{\theta}$ and verifies that $\iota(\nu)<\iota\left(\nu_{0}\right)$ for all $\nu_{0} \neq \nu \in D_{\theta}$. Lemma 1.13 implies that $\lim _{T \rightarrow \infty} \tilde{G}_{\theta}=\lim _{T \rightarrow \infty} \prod_{i=1}^{r} \mathbf{L}^{-\kappa\left(v_{i}\right)} T^{\iota\left(v_{i}\right)}\left(1-\mathbf{L}^{-\kappa\left(v_{i}\right)} T^{\iota\left(v_{i}\right)}\right)^{-1}$. The result follows from Lemma 1.12 .

Let $\theta \subset \mathbf{R}^{m}$ be a rational simplicial cone of dimension $r$. We denote by $v_{1}, \ldots, v_{r} \in \mathbf{Z}^{m}$ the primitive vectors generating the edges of the cone $\theta$ and by $\mathbf{Z}\left(\theta \cap \mathbf{Z}^{m}\right)$ the sublattice of $\mathbf{Z}^{m}$ spanned by $\theta \cap \mathbf{Z}^{m}$. The lattice $\oplus_{i=1}^{r} \mathbf{Z} v_{i}$ is a sublattice of finite index mult $(\theta)$ of $\mathbf{Z}\left(\theta \cap \mathbf{Z}^{m}\right)$. Notice that $\operatorname{mult}(\theta)$ is equal to the cardinality of the set $D_{\theta}$ introduced in Lemma 3.2

Notation 3.3. If $\theta \in \Theta_{j}$, for $1 \leq j \leq g+1$, is a simplicial cone we denote by $J_{\theta}(f, s)$ the element of $\mathbf{Q}(s)$ given by $J_{\theta}(f, s):=\operatorname{mult}(\theta) \prod_{v}\left(\xi_{j}(v)+\eta(v) s\right)^{-1}$, where $v$ runs through the primitive vectors defining the edges of $\theta$. If $\theta \in \Theta_{j}$ is not simplicial then $\theta=\sigma_{j}^{-}$and we set $J_{\sigma_{j}^{-}}(f, s):=\left(1+n_{j} \ldots n_{g} s\right)^{-1}\left(J_{\rho_{j-1}}(f, s)-J_{\sigma_{j}^{+}}(f, s)\right)$, where $J_{\rho_{0}}(f, s):=1$.

Notation 3.4. We use the notations introduced in Lemma 3.1] Let $\theta$ be a cone in $\Theta_{j}$, for some $j=1, \ldots, g+1$. We denote by $\tilde{P}_{\theta}$ the image of $P_{\theta}$ by the monomial mapping

$$
\mathbf{Z}\left[x_{1}, \ldots, x_{d+g+1}\right] \rightarrow \mathbf{Z}[\mathbf{L}, T], \quad x^{\nu} \mapsto \mathbf{L}^{-\xi_{j}(\nu)} T^{\eta(\nu)} .
$$

We define the rational function

$$
S_{\theta}:=\tilde{P}_{\theta} \prod_{v}\left(1-\mathbf{L}^{-\xi_{j}(v)} T^{\eta(\nu)}\right)^{-1}
$$

where $v$ run through the primitive integral vectors in the edges of $\theta$.

Remark 3.5. The rational function $S_{\theta}$ is well defined. By Lemma 2.22 for any vector $v$ as above we have that $\left(\xi_{j}(v), \eta(v)\right) \neq(0,0)$.

Definition 3.6. If $\theta \in \Theta_{j}$ for $1 \leq j \leq g$, we define the elements $c_{1}(\theta) \in K_{0}^{\hat{\mu}}$ (Var $\mathbf{C}$ ) (resp. $\left.c(\theta) \in K_{0}\left(\operatorname{Var}_{\mathbf{C}}\right)\right)$ as

$$
\begin{cases}{\left[\mu_{n_{j} e_{j}}\right] \quad(\text { resp. } \mathbf{L}-1)} & \text { if } \theta=\sigma_{j}^{+}, \\ {\left[\left\{(x, y) \in\left(\mathbf{C}^{*}\right)^{2} \mid\left(y^{n_{j}}-x^{r_{j}}\right)^{e_{j}}=1\right\}\right] \quad(\text { resp. }(\mathbf{L}-1)(\mathbf{L}-2))} & \text { if } \theta=\rho_{j}, \\ {\left[\mu_{r_{j} e_{j}}\right] \quad(\text { resp. } \mathbf{L}-1)} & \text { if } \theta=\sigma_{j}^{-} .\end{cases}
$$

If $\theta=\sigma_{g+1} \in \Theta_{g+1}$ we set $c_{1}(\theta):=1($ resp. $c(\theta):=\mathbf{L}-1)$.

Theorem 3.7. Let $f:\left(\mathbf{C}^{d+1}, 0\right) \rightarrow(\mathbf{C}, 0)$ be the complex analytic function defined by an irreducible q.o. polynomial. The zeta functions $Z(f, T)_{0}, Z^{\text {naive }}(f, T)_{0}, Z_{\mathrm{top}}(f, s)_{0}$ and the motivic Milnor fibre $\mathcal{S}_{f, 0}$ of the $f$ at 0 are determined by the embedded topological type of the q.o. hypersurface germ $(S, 0) \subset\left(\mathbf{C}^{d+1}, 0\right)$ defined by $\{f=0\}$. We have the following formulas in terms of 
the characteristic exponents of the q.o. branch $\zeta$ parametrizing $(S, 0)$ :

$$
\begin{gathered}
Z_{\text {top }}(f, s)_{0}=J_{\sigma_{g+1}}(f, s)+\sum_{j=1}^{g}\left(J_{\sigma_{j}^{+}}(f, s)-J_{\rho_{j}}(f, s)+J_{\sigma_{j}^{-}}(f, s)\right) \\
Z^{\text {naive }}(f, T)_{0}=\sum_{j=1}^{g+1} \sum_{\theta \in \Theta_{j}} c(\theta)(\mathbf{L}-1)^{\operatorname{dim} \theta-1} S_{\theta}, \\
Z(f, T)_{0}=\sum_{j=1}^{g+1} \sum_{\theta \in \Theta_{j}} c_{1}(\theta)(\mathbf{L}-1)^{\operatorname{dim} \theta-1} S_{\theta}, \\
\mathcal{S}_{f, 0}=(1-\mathbf{L})^{\ell_{g}}+\sum_{j=1}^{g}\left((1-\mathbf{L})^{\ell_{j}-1}\left(c_{1}\left(\rho_{j}\right)+c_{1}\left(\sigma_{j}^{+}\right)\right)+(1-\mathbf{L})^{\ell_{j-1}} c_{1}\left(\sigma_{j}^{-}\right)\right)
\end{gathered}
$$

Remark 3.8. For $d=1$ we recover a reformulation of results of Guibert (see [20], Proposition 3.3.1) and Artal et al. (see [3], Theorem 4.3, 4.9 and 5.3). Notice that Guibert asssumes that the line $\{x=0\}$ is non-tangent to the curve.

Remark 3.9. If $g=1$ then $f$ is Newton non-degenerate and the formula above for $Z_{\text {top }}(f, s)_{0}$ reformulates in this particular case the one given by Denef and Loeser (see Théorème 5.3 [5]).

Remark 3.10. If $d \geq 2$ the phenomenon of false reducibility, discovered in [19, may lead to inaccurate formulas for the zeta functions of q.o. singularities in [3] (see Example [10.1). In Section 9 we compare the method of Newton maps used in [3] with the toric methods used in this paper. We check that the sets of candidate poles we get below (see Corollary 3.17) is the same as the one given in [3].

Corollary 3.11. With the hypothesis of Theorem 3.7 the Hodge-Steenbrink spectrum of $f$ at 0 is determined by the characteristic exponents. We have the formula:

$$
S p^{\prime}(f, 0)=(-1)^{d}\left(\sum_{j=1}^{g}\left((1-t)^{\ell_{j}-1}\left(P_{n_{j}, r_{j}}^{\left(e_{j}\right)}+P_{n_{j} e_{j}}\right)+(1-t)^{\ell_{j-1}} P_{r_{j} e_{j}}\right)+(1-t)^{\ell_{g}}-1\right) .
$$

Proof. It follows by using the formula for the motivic Milnor fiber in Theorem 3.7. Notation 1.6. Lemma 1.5 and Corollary 1.7

By Corollary 3.11 the Hodge-Steenbrink spectrum of an irreducible q.o. polynomial $f$ is determined by the embedded topological type of the germ of singularity defined by $f=0$.

Remark 3.12. For $d=1$ we recover Saito's Formula ([36] Theorem 1.5 or [20], Corollary 3.4.1). See Remark 1.9. In this case the Hodge-Steenbrink spectrum of $f$ at 0 classifies the embedded topological type of the analytically irreducible germ of plane curve $(S, 0) \subset\left(\mathbf{C}^{2}, 0\right)$ defined by $f=0$. However, there are examples of functions $f_{i}:\left(\mathbf{C}^{2}, 0\right) \rightarrow(\mathbf{C}, 0)$ defining non-irreducible germs at the origin such that the Hodge-Steenbrink spectrum of $f_{i}$ at 0 is the same for $i=1,2$ meanwhile the germs defined by $f_{i}=0, i=1,2$ have different embedded topology, see [41, 38].

Remark 3.13. If $d \geq 2$ the Hodge-Steenbrink spectrum associated to an irreducible q.o. polynomial $f \in \mathbf{C}\left\{x_{1}, \ldots, x_{d}\right\}[y]$ is not a complete invariant of the embedded topological type of the germ $f=0$ at the origin. For instance, the q.o. polynomials $f_{1}:=y^{3}-x_{1} x_{2}$ and $f_{2}:=y^{3}-x_{1}^{2} x_{2}$ define irreducible germs with different embedded topological types meanwhile Corollary 3.11 implies their Hodge-Steenbrink spectrums coincide.

Remark 3.14. If $d \geq 3$ the Seifert form on the middle homology of the Milnor fiber of an hypersurface singularity $F:\left(\mathbf{C}^{d+1}, 0\right) \rightarrow(\mathbf{C}, 0)$ defining an isolated singularity at the origin determines the topological type of the singularity (see [12], Theorem 3.1). However, there are examples of germs $f_{i}:\left(\mathbf{C}^{2}, 0\right) \rightarrow(\mathbf{C}, 0)$ defining non-irreducible germs at the origin such that they have isomorphic integral Seifert form but the germs defined by $f_{1}=0$ and $f_{2}=0$ have different embedded topology and different spectrum (see [11]). The germs $F_{i}:\left(\mathbf{C}^{4}, 0\right) \rightarrow(\mathbf{C}, 0)$ given by $F_{i}=f_{i}\left(x_{1}, x_{2}\right)+x_{3}^{2}+x_{4}^{2}, i=1,2$, have isomorphic Seifert forms (see [37]). It follows that the germs $F_{i}=0$ at $0 \in \mathbf{C}^{4}$ have the same embedded topological type, meanwhile the application of Thom Sebastiani Theorem for the spectrum implies that $F_{1}$ and $F_{2}$ have different 
spectrum at 0 (see [8] Theorem 5.1 or [42]). In this example the topological zeta functions of $F_{i}$ at $0, i=1,2$, are also different (see [2]).

Definition 3.15. (see Notations 2.19] and 2.5) The vector $\nu_{i}^{(j)} \in \rho_{j}$ is special if for any $1 \leq i^{\prime} \leq d$ the following equalities

$$
\lambda_{j^{\prime}, i^{\prime}}=0 \text { for } 1 \leq j^{\prime}<j \text {, and } \lambda_{j, i^{\prime}}=1 / n_{j},
$$

are verified iff $i^{\prime}=i$, and in addition we have that $\lambda_{j+1, i}>\lambda_{j, i}$ if $j<g$.

Example 3.16. If $\left\{x_{i}=0\right\} \cap S$ is the unique coordinate hyperplane section of $(S, 0)$ which is not contained in the singular locus of $(S, 0)$ but it is contained in the critical locus of the quasiordinary projection $\pi$, then $\nu_{i}^{(j)}$ is a special vector. This is a consequence of the characterization of the singular locus of a q.o. hypersurface (see [26. Therorem 7.3]).

Corollary 3.17. (compare with [3, Definition 5.5 and Proposition 5.7]) With the hypothesis of Theorem 3.7 and the Notation 2.19 there exists a polynomial $Q \in \mathbf{Z}[$ s] such that

$$
Z(f, s)_{0}=Q(1+s)^{-1} \prod_{j=1}^{g} \prod_{\nu_{i}^{(j)} \text { non-special }}\left(\xi_{j}\left(\nu_{i}^{(j)}\right)+\eta\left(\nu_{i}^{(j)}\right) s\right)^{-1} .
$$

There exists $R \in \mathbf{Z}\left[\mathbf{L}^{ \pm 1}\right][T]$ such that

$$
Z^{\text {naive }}(f, T)_{0}=R\left(1-\mathbf{L}^{-1} T\right)^{-1} \prod_{j=1}^{g} \prod_{\nu_{i}^{(j)} \text { non-special }}^{i=1, \ldots, d}\left(1-\mathbf{L}^{-\xi_{j}\left(\nu_{i}^{(j)}\right)} T^{\eta\left(\nu_{i}^{(j)}\right)}\right)^{-1} .
$$

Notice that if $\nu_{i}^{(j)}=\epsilon_{i}$ then it does not contribute to a pole of these zeta functions.

Remark 3.18. We prove Corollary 3.17 in Section 9. We can use Corollary 3.17, Remark 9.16] and the arguments from [3, Chapter 6] to complete the proof of the monodromy conjecture in this case. The argument consists of showing that the candidate poles are related with monodromy eigenvalues associated to the restriction of $f$ to suitable plane sections of $\mathbf{C}^{d+1}$ in a neighbourhood of 0 .

In order to prove these results we use an embedded resolution $\Pi^{\prime}: Z^{\prime} \rightarrow \mathbf{C}^{d+1}$ of $(S, 0) \subset$ $\left(\mathbf{C}^{d+1}, 0\right)$. This resolution is a composition of toric modifications which we recall in Section 5 following [17. We remark a relation between the denominators of the zeta functions in Corollary 3.17 and certain exceptional divisors of the modification $\Pi^{\prime}$.

Remark 3.19. For any $1 \leq i \leq d$ and $1 \leq j \leq g$ there exists an irreducible divisor $E_{i}^{(j)} \subset Z^{\prime}$ such that the order of vanishing of the jacobian of $\Pi^{\prime}$ (resp. of $f \circ \Pi^{\prime}$ ) along an irreducible divisor $E_{i}^{(j)} \subset Z^{\prime}$ is equal to $\xi_{j}\left(\nu_{i}^{(j)}\right)-1$ (resp. $\eta\left(\nu_{i}^{(j)}\right)$. Notice that $E_{i}^{(j)}$ is contained in the critical locus of $\Pi^{\prime}$, that is $E_{i}^{(j)}$ is exceptional divisor for $\Pi^{\prime}$, if and only if $\xi_{j}\left(\nu_{i}^{(j)}\right)>1$. These facts are consequence of Remarks 7.4 and 7.5 below.

\section{Some notions On TORIC GEOMETRY AND NEWTON POLYHEDRA}

In this section we introduce the notations and basic results of toric geometry which we use in this paper (see [14, 13, 23, 31]).

If $N \cong \mathbf{Z}^{d}$ is a lattice we denote by $N_{\mathbf{R}}$ the vector space spanned by $N$ over the field $\mathbf{R}$. In what follows a cone mean a rational convex polyhedral cone: the set of non negative linear combinations of vectors $v_{1}, \ldots, v_{r} \in N$. The cone $\tau$ is strictly convex if it contains no lines, in such case we denote by 0 the 0 -dimensional face of $\tau$. The dimension of $\tau$ is the dimension the linear span of $\tau$ in $N_{\mathbf{R}}$. The relation $\theta \leq \tau$ (resp. $\theta<\tau$ ) denotes that $\theta$ is a face of $\tau$ (resp. $\theta \neq \tau$ is a face of $\tau$ ). The cone $\tau$ is simplicial the number of edges of $\tau$ is equal to the dimension of $\tau$. The cone $\tau$ is regular if it is generated by a sequence of vectors $v_{1}, \ldots, v_{r}$ which is contained in a basis of the lattice $N$.

We denote by $M$ the dual lattice, i.e., $M=\operatorname{Hom}(N, \mathbf{Z})$ and by $\langle\rangle:, N \times M \rightarrow \mathbf{Z}$ the duality pairing between the lattices $N$ and $M$. The dual cone $\tau^{\vee}$ (resp. orthogonal cone $\tau^{\perp}$ ) of $\tau$ is the 
set $\left\{w \in M_{\mathbf{R}} \mid\langle u, w\rangle \geq 0\right.$, (resp. $\left.\left.\langle u, w\rangle=0\right) \quad \forall u \in \tau\right\}$. We denote the relative interior of the cone $\tau$ by $\stackrel{\circ}{\tau}$ (or also by $\operatorname{int}(\tau)$ when it is more convenient for typographical reasons).

A fan $\Sigma$ is a family of strictly convex cones in $N_{\mathbf{R}}$ such that for any $\sigma, \sigma^{\prime} \in \Sigma$ we have $\sigma \cap \sigma^{\prime} \in \Sigma$ and if $\tau \leq \sigma$ then $\tau \in \Sigma$. The support of the fan $\Sigma$ is the set $|\Sigma|:=\bigcup_{\tau \in \Sigma} \tau \subset N_{\mathbf{R}}$. We say that a fan $\Sigma^{\prime}$ is a subdivision of the fan $\Sigma$ if both fans have the same support and if every cone of $\Sigma^{\prime}$ is contained in a cone of $\Sigma$. The fan $\Sigma^{\prime}$ is a regular subdivision of $\Sigma$ if all the cones in $\Sigma^{\prime}$ are regular and $\theta \in \Sigma^{\prime}$ for any regular cone $\theta \in \Sigma$. Every fan admits a regular subdivision.

Let $\sigma$ be an strictly convex cone rational for the lattice $N$. By Gordan's Lemma the semigroup $\sigma^{\vee} \cap M$ is finitely generated. We denote by $\mathbf{C}\left[\sigma^{\vee} \cap M\right]:=\left\{\sum_{\text {finite }} c_{\delta} x^{\delta} \mid c_{\delta} \in \mathbf{C}, \delta \in \sigma^{\vee} \cap M\right\}$ the semigroup algebra of $\sigma^{\vee} \cap M$ with complex coefficients. The toric variety $Z_{\sigma}:=\operatorname{Spec} \mathbf{C}\left[\sigma^{\vee} \cap M\right]$, denoted also by $Z_{\sigma, N}$ or $Z^{\sigma^{\vee} \cap M}$, is normal. The torus $T_{N}:=Z^{M}$ is an open dense subset of $Z_{\sigma}$, which acts on $Z_{\sigma}$ and the action extends the action of the torus on itself by multiplication. There is a one to one correspondence between the faces $\tau$ of $\sigma$ and the orbits $\operatorname{orb}_{\tau}$ of the torus action on $Z_{\sigma}$, which reverses the inclusions of their closures.

The affine varieties $Z_{\sigma}$ corresponding to cones in a fan $\Sigma$ glue up to define a toric variety $Z_{\Sigma}$. A subdivision $\Sigma^{\prime}$ of the fan $\Sigma$ defines a toric modification $\phi: Z_{\Sigma^{\prime}} \rightarrow Z_{\Sigma}$. If $\Sigma^{\prime}$ is a regular subdivision of $\Sigma$ then the modification $\phi$ is a resolution of singularities of $Z_{\Sigma}$.

If $\theta$ is $d$-dimensional strictly convex cone then its orbit $\operatorname{orb}_{\theta}$ is reduced to a closed point called the origin 0 of the toric variety $Z_{\theta}$. We denote by $\mathbf{C}\left[\left[\theta^{\vee} \cap M\right]\right]$ the ring of formal power series with exponents in $\tau^{\vee} \cap M$. This ring is the completion with respect to the maximal ideal of the ring $\mathbf{C}\left\{\theta^{\vee} \cap M\right\} \subset \mathbf{C}\left[\left[\theta^{\vee} \cap M\right]\right]$ of germs of holomorphic functions at $0 \in Z_{\tau}$. The Newton polyhedron $\mathcal{N}(\psi)$ of a nonzero series $\psi=\sum c_{\delta} x^{\delta} \in \mathbf{C}\left[\left[\theta^{\vee} \cap M\right]\right]$ is the convex hull of the Minkowski sum $\left\{\delta \mid c_{\delta} \neq 0\right\}+\theta^{\vee}$. The support function of the polyhedron $\mathcal{N}(\psi)$ is the piecewise linear function

$$
h: \theta \rightarrow \mathbf{R}, \quad \nu \mapsto \inf \{\langle\nu, \omega\rangle \mid \omega \in \mathcal{N}(\psi)\} .
$$

A vector $\nu \in \theta$ defines the face $\mathcal{F}_{\nu}:=\{\omega \in \mathcal{N}(\psi) \mid\langle\nu, \omega\rangle=h(\nu)\}$ of the polyhedron $\mathcal{N}(\psi)$. All faces of $\mathcal{N}(\psi)$ are of this form, in particular, the compact faces are defined by vectors $\nu \in \stackrel{\circ}{*}$. The set consisting of the cones $\sigma(\mathcal{F}):=\{\nu \in \theta \mid\langle\nu, \omega\rangle=h(\nu), \forall \omega \in \mathcal{F}\}$, for $\mathcal{F}$ running through the faces of $\mathcal{N}(\psi)$ is a fan subdividing the cone $\theta$. If $\nu \in \theta$ we denote by $\psi_{\mid \nu}$ the symbolic restriction $\psi_{\mid \nu}:=\sum_{\delta \in \mathcal{F}_{\nu}} c_{\delta} x^{\delta}$ of the series $\psi$ to the face $\mathcal{F}_{\nu}$ of its Newton polyhedron. If $\nu \in \stackrel{\circ}{\nu}$ then $\psi_{\mid \nu}$ is a polynomial, i.e., $\psi_{\mid \nu} \in \mathbf{C}\left[\theta^{\vee} \cap M\right]$.

\section{TORIC EMBEDded RESOlutions OF Q.O. HYPERSURFACES}

In this section we give an outline of the construction of a toric embedded resolution of a q.o. hypersurface following [17].

Notation 5.1. Recall that we denote by $\rho$ the cone $\mathbf{R}_{>0}^{d} \subset N_{\mathbf{R}}$ (see Notation 2.16). We denote by $N_{j}^{\prime}$ the lattice $N_{j} \times \mathbf{Z}$, and by $M_{j}^{\prime}$ its dual lattice $M_{j} \times \mathbf{Z}$, for $j=0, \ldots, g$. The cone $\rho^{\prime}:=\rho \times \mathbf{R}_{\geq 0}$ is rational for the lattice $N_{j}^{\prime}$.

We keep notations of Section 2 that is, $\zeta$ is a q.o. branch with characteristic exponents $\lambda_{1}, \ldots, \lambda_{g}$. The q.o. branch $\zeta$ parametrizes a q.o. hypersurface $(S, 0)$ defined by a q.o. polynomial $f \in \mathbf{C}\left\{x_{1}, \ldots, x_{d}\right\}[y]$. We assume, making a change of coordinates, that the coordinate $y$ is equal to $f_{0}$ (see Definition [2.8).

With this assumptions the Newton polyhedron $\mathcal{N}(f) \subset M_{\mathbf{R}}^{\prime}$ has only one compact edge $\mathcal{E}_{1}$ with vertices $\left(0, e_{0}\right)$ and $\left(e_{0} \lambda_{1}, 0\right)$. It defines a subdivision $\Sigma_{1}$ of the cone $\rho^{\prime}$, with only two $(d+1)$-dimensional cones $\bar{\sigma}_{1}^{+}$and $\bar{\sigma}_{1}^{-}$which intersect along the $d$-dimensional face $\bar{\rho}_{1}$. The support function of the Newton polyhedron $\mathcal{N}(f)$ is defined for $(\nu, r) \in \rho^{\prime}$ by:

$$
h^{(1)}(\nu, r)= \begin{cases}e_{1}\left\langle\nu, n_{1} \lambda_{1}\right\rangle & \text { if } \quad(\nu, r) \in \bar{\sigma}_{1}^{+}, \\ e_{1} n_{1} r & \text { if } \quad(\nu, r) \in \bar{\sigma}_{1}^{-},\end{cases}
$$

that is, the cone $\bar{\sigma}_{1}^{-}$(resp. $\left.\bar{\sigma}_{1}^{+}\right)$corresponds to the vertex $\left(0, e_{0}\right)$ (resp. $\left.\left(e_{0} \lambda_{1}, 0\right)\right)$ of $\mathcal{N}(f)$. The polynomial $f$ is of the form,

$$
f=\left(y^{n_{1}}-c_{1} x^{n_{1} \lambda_{1}}\right)^{e_{1}}+\cdots
$$


where $c_{1} \in \mathbf{C}^{*}$ and the terms which are not written lie above the edge $\mathcal{E}_{1}$.

Remark 5.2. We choose the notation $\bar{\sigma}_{1}^{+}, \bar{\sigma}_{1}^{-}$and $\bar{\rho}_{1}$ for the cones in $\Sigma_{1}$ since these cones are related with the cones $\sigma_{1}^{+}, \sigma_{1}^{-}$and $\rho_{1}$ in $\Theta_{1}$ (see Definition 2.20 and the proof of Theorem 6.11).

We describe the transformation of the hypersurface $(S, 0)$ under the toric modification

$$
\Pi_{1}: Z_{1} \rightarrow Z_{0}:=\mathbf{C}^{d+1}
$$

defined by the subdivision $\Sigma_{1}$. Notice that $Z_{1}$ is not a smooth variety. First we need an elementary lemma to describe the chart $Z_{\bar{\rho}_{1}, N_{0}^{\prime}} \subset Z_{1}$ associated to the cone $\bar{\rho}_{1}$ and the lattice $N_{0}^{\prime}$.

Lemma 5.3. Let us consider the lattice homomorphism

$$
\phi_{1}: M_{0}^{\prime} \rightarrow M_{1}, \quad(\lambda, a) \mapsto \lambda+a \lambda_{1} .
$$

We have the following properties:

(i) The homomorphism $\phi_{1}$ is surjective.

(ii) $\operatorname{ker}\left(\phi_{1}\right) \cap M_{0}^{\prime}=\bar{\rho}_{1}^{\perp} \cap M_{0}^{\prime}=\left(-n_{1} \lambda_{1}, n_{1}\right) \mathbf{Z}$.

(iii) $\phi_{1}^{-1}\left(\rho^{\vee} \cap M_{1}\right)=\bar{\rho}_{1}^{\vee} \cap M_{0}^{\prime}$.

(iv) We have an isomorphism of semigroups $\bar{\rho}_{1}^{\vee} \cap M_{0}^{\prime} \rightarrow\left(\rho^{\vee} \cap M_{1}\right) \times \mathbf{Z}$.

(iv) The dual homomorphism $\phi_{1}^{*}: N_{1} \rightarrow N_{0}^{\prime}$ is injective and verifies that $\phi_{1}^{*}(\nu)=\left(\nu,\left\langle\nu, \lambda_{1}\right\rangle\right)$.

(v) $\phi_{1}^{*}\left(N_{1}\right)$ is the lattice generated by $\bar{\rho}_{1} \cap N_{0}^{\prime}$.

(vi) $\phi_{1}^{*}\left(\rho \cap N_{1}\right)=\bar{\rho}_{1} \cap N_{0}^{\prime}$.

Proof. Recall that by definition we have the equality $N_{1}=\left\{\nu \in N_{0} \mid\left\langle\nu, \lambda_{1}\right\rangle \in \mathbf{Z}\right\}$. The details of the proof are left to the reader (see [17] Lemma 17).

By Lemma 5.3 the term

$$
w_{1}:=y^{n_{1}} x^{-n_{1} \lambda_{1}}
$$

is a holomorphic function on the chart $Z_{\bar{\rho}_{1}, N_{0}^{\prime}}$.

Lemma 5.4. We have the following properties:

(i) The orbit $\operatorname{orb}\left(\bar{\rho}_{1}\right)$ is a one-dimensional torus embedded as a closed subset in the chart $Z_{\bar{\rho}_{1}, N_{0}^{\prime}}$.

(ii) The coordinate ring of $\operatorname{orb}\left(\bar{\rho}_{1}\right)$ is equal to $\mathbf{C}\left[w_{1}^{ \pm 1}\right]$.

(iii) The hypersurface $\left\{w_{1}=c\right\} \subset Z_{\bar{\rho}_{1}, N_{0}^{\prime}}$ is isomorphic to $Z_{\rho, N_{1}}$ for any $c \in \mathbf{C}^{*}$.

(iv) The chart $Z_{\bar{\rho}_{1}, N_{0}^{\prime}}$ is isomorphic to $Z_{\rho, N_{1}} \times \mathbf{C}^{*}$.

(v) If o $\in \operatorname{orb}\left(\bar{\rho}_{1}\right)$ the germ $\left(Z_{\bar{\rho}_{1}, N_{0}^{\prime}}, o\right)$ is isomorphic to $\left(Z_{\rho, N_{1}} \times \mathbf{C},(0,0)\right)$.

Proof. The first assertion is well-known. The statement (ii) is consequence of (ii) in Lemma 5.3. The assertion (iv) is consequence of the isomorphism of semigroups in Lemma 5.3 (iv). The statement (iii) is consequence of (iv) and (ii). The assertion (v) is deduced from (iv).

The modification $\Pi_{1}$ is an isomorphism over the torus $T_{N_{0}^{\prime}}=\left(\mathbf{C}^{*}\right)^{d+1} \subset Z_{0}$. The strict transform $S^{(1)}$ of the hypersurface $S$ by $\Pi_{1}$ is the closure of $\Pi_{1}^{-1}\left(S \cap\left(\mathbf{C}^{*}\right)^{d+1}\right)$ in $Z_{1}$. It is shown in 17] that $S^{(1)}$ intersects the exceptional fiber $\Pi_{1}^{-1}(0)$ only at one point $o_{1}$ which belongs to the orbit $\operatorname{orb}\left(\bar{\rho}_{1}\right)$ of $Z_{1}$. Hence, in the chart $Z_{\bar{\rho}_{1}}=Z_{\bar{\rho}_{1}, N_{0}^{\prime}}$ we can factorize

$$
\left(f \circ \Pi_{1}\right)_{\mid Z_{\bar{\rho}_{1}}}=x^{e_{1} n_{1} \lambda_{1}}\left(\left(w_{1}-c_{1}\right)^{e_{1}}+\cdots\right),
$$

where the terms which are not written vanish on $\operatorname{orb}\left(\bar{\rho}_{1}\right)$. The strict transform of $(S, 0)$ by $\Pi_{1}$ is a hypersurface germ $\left(S^{(1)}, o_{1}\right) \subset\left(Z_{\bar{\rho}_{1}, N_{0}^{\prime}}, o_{1}\right)$ defined by the vanishing of $\left(x^{-e_{1} n_{1} \lambda_{1}} \cdot f \circ \Pi_{1}\right)_{\mid Z_{\bar{\rho}_{1}}}$. The intersection $S^{(1)} \cap \operatorname{orb}\left(\bar{\rho}_{1}\right)$, defined by the equation $\left(w_{1}-c_{1}\right)^{e_{1}}=0$, consists of the point $o_{1}$ counted with multiplicity $e_{1}$. We get that the strict transform $S_{1}^{(1)}$ of the semi-root $S_{1}$ intersects the orbit $\operatorname{orb}\left(\bar{\rho}_{1}\right)$ at the point $o_{1}$ with multiplicity one. It follows that the function

$$
y_{1}:=x^{-n_{1} \lambda_{1}} \cdot\left(f_{1} \circ \Pi_{1}\right) \mid Z_{\bar{\rho}_{1}},
$$

can be taken as a local coordinate at $o_{1}$, replacing $w_{1}-c_{1}$. By Lemma 5.4 (iii) the strict transform $S_{1}^{(1)}$ is analytically isomorphic to the germ of toric variety $Z_{\rho, N_{1}}$ at the origin. Taking into account the isomorphism of Lemma 5.4 (iv) we get. 
Proposition 5.5. 17, Proposition 19] Let us consider the projection

$$
\pi_{1}: Z_{\rho, N_{1}} \times \mathbf{C}^{*} \rightarrow Z_{\rho, N_{1}} .
$$

The restriction of $\pi_{1 \mid S^{(1)}}:\left(S^{(1)}, o_{1}\right) \rightarrow\left(Z_{\rho, N_{1}}, 0\right)$ defines an unramified finite covering of the torus $T_{N_{1}} \subset Z_{\rho, N_{1}}$.

Remark 5.6. Proposition 5.5 means that the strict transform $\left(S^{(1)}, o_{1}\right)$ is a germ of toric quasiordinary (t.q.o.) hypersurface relative to the base $Z_{\rho, N_{1}}$ (see [17, Definition 5 and [16]). If the cone $\rho$ is regular for the lattice $N_{1}$ then $\left(S^{(1)}, o_{1}\right)$ is a q.o. hypersurface in the classical sense. The notions and results in Section 2 generalize to the case of t.q.o. singularities in the relative hypersurface case. This applies in particular for the definition of characteristic exponents, semigroup, semi-roots, etc. It is convenient to consider the characteristic exponents of a (classical) q.o. branch $\zeta$ not only as $d$-tuples of rational numbers, but as rational vectors inside a reference cone $\rho^{\vee}$, which is regular for a reference lattice $M_{0}$. Then, if we drop the regularity condition, the statement of Lemma 2.2 characterizes the t.q.o. branches, which parametrize the t.q.o. singularities in the relative hypersurface case. See [17. The arguments above apply similarly to the semiroots $S_{j}^{(1)}$.

Proposition 5.7. 17, Proposition 19] The following statements hold for $j=2, \ldots, g$.

(i) The strict transform $\left(S_{j}^{(1)}, o_{1}\right)$ of the semi-root $\left(S_{j}, 0\right)$ is parametrized by a t.q.o. branch $\zeta_{j}^{(1)}$ which has characteristic exponents $\lambda_{2}-\lambda_{1}, \ldots, \lambda_{j}-\lambda_{1}$ and characteristic integers $n_{2}, \ldots, n_{g}$, with respect to the reference cone $\rho^{\vee}$ and reference lattice $M_{1}$.

(ii) The germ $\left(S_{j}^{(1)}, o_{1}\right)$ is a $(j-1)$-th semi-root of $\left(S^{(1)}, o_{1}\right)$.

If $g>1$ it follows from Propositions 5.5 and 5.7 that the germ $\left(S_{j}^{(1)}, o_{1}\right)$ is defined by the vanishing of

$$
f_{j}^{(1)}:=\left(x^{-n_{1} \ldots n_{j} \lambda_{1}} f_{j} \circ \Pi_{1}\right)_{\mid Z_{\bar{\rho}_{1}}} .
$$

The germ $\left(S_{j}^{(1)}, o_{1}\right)$ is parametrized by setting $y_{1}=\zeta_{j}^{(1)}$, for $j=2, \ldots, g$. We can expand $f_{j}^{(1)}$ as an element of $\mathbf{C}\left\{\left(\rho^{\prime}\right)^{\vee} \cap M_{1}^{\prime}\right\}$ in such a way that its Newton polyhedron $\mathcal{N}\left(f^{(1)}\right) \subset\left(M_{1}\right)_{\mathbf{R}} \times \mathbf{R}$ has a unique compact edge $\mathcal{E}_{2}$ with vertices $\left(e_{1}\left(\lambda_{2}-\lambda_{1}\right), 0\right)$ and $\left(0, e_{1}\right)$. This expansion of $f^{(1)}$ is of the form,

$$
\left.f^{(1)}=\left(y_{1}^{n_{2}}-c_{2} x^{n_{2}\left(\lambda_{2}-\lambda_{1}\right.}\right)\right)^{e_{2}}+\cdots
$$

where $c_{2} \in \mathbf{C}^{*}$ and the terms which are not written lie above the edge $\mathcal{E}_{2}$. The polyhedron $\mathcal{N}\left(f^{(1)}\right)$ defines a subdivision $\Sigma_{2}$ of $\rho^{\prime}$ with only two cones of dimension $d+1$. These cones are $\bar{\sigma}_{2}^{+}$(resp. $\left.\bar{\sigma}_{2}^{-}\right)$, which corresponds to the vertex $\left(e_{1}\left(\lambda_{2}-\lambda_{1}\right), 0\right)$ (resp. $\left.\left(0, e_{1}\right)\right)$ of the polyhedron $\mathcal{N}\left(f^{(1)}\right)$. With respect to these new coordinates we consider the toric modification $\Pi_{2}: Z_{3} \rightarrow Z_{2}$, induced by the subdivision $\Sigma_{2}$. We set $w_{2}:=y_{1}^{n_{2}} x^{-n_{2}\left(\lambda_{2}-\lambda_{1}\right)}$. In the chart $Z_{\bar{\rho}_{2}}=Z_{\bar{\rho}_{2}, N_{1}^{\prime}} \subset Z_{3}$ one can factorize

$$
\left(f^{(1)} \circ \Pi_{2}\right)_{\mid Z_{\bar{\rho}_{2}}}=x^{e_{1}\left(\lambda_{2}-\lambda_{1}\right)}\left(\left(w_{2}-c_{2}\right)^{e_{3}}+\cdots\right),
$$

where the terms which are not written vanish on $\operatorname{orb}\left(\bar{\rho}_{2}\right)$. The strict transform $S^{(2)}$, defined then by the vanishing of $x^{-e_{1}\left(\lambda_{2}-\lambda_{1}\right)} \cdot\left(f^{(1)} \circ \Pi_{2}\right)$, is a germ at the point $o_{2} \in \operatorname{orb}\left(\bar{\rho}_{2}\right)$ given by $w_{2}-c_{2}=0$.

Then, we iterate this procedure, obtaining for $2<j \leq g$ a sequence of local toric modifications $\Pi_{j-1}: Z_{j} \rightarrow Z_{j-1}$, which are described by replacing the index 1 by $j-1$ and the index 2 by $j$ above. The toric modification $\Pi_{j-1}$ is defined in terms of the fan $\Sigma_{j-1}$ which subdivides the cone $\rho^{\prime}$, with respect to the lattice $N_{j-1}^{\prime}=N_{j-1} \times \mathbf{Z}$, into cones $\bar{\sigma}_{j}^{+}, \bar{\sigma}_{j}^{+}$and $\bar{\rho}_{j}$ for $j=1, \ldots, g$. We denote by $\Sigma_{g+1}$ the fan of faces of the cone $\rho^{\prime}$, with lattice $N_{g}^{\prime}$.

Lemma 5.8. We have the following equalities for $1 \leq j \leq g$,

$$
\left(f_{j} \circ \Pi_{1} \circ \cdots \circ \Pi_{j}\right)_{\mid Z_{\bar{\rho}_{j}}}=x^{n_{j} \gamma_{j}} y_{j} \quad \text { and } \quad\left(f \circ \Pi_{1} \circ \cdots \circ \Pi_{j}\right)_{\mid Z_{\bar{\rho}_{j}}}=x^{e_{j-1} \gamma_{j}} f^{(j)} .
$$

Proof. This is consecuence of the decompositions (8), (9) and (11) at levels $2, \ldots, j$, together with Lemma 2.15 




FIGURE 1.

Remark 5.9. The strict transform $\left(S^{(g)}, o_{g}\right)$ of $(S, 0)$ by the composition $\Pi_{1} \circ \cdots \circ \Pi_{g}$ is isomorphic to the germ of toric variety $\left(Z_{\rho, N}, 0\right)$, with $N=N_{g}$. This germ is the normalization of $(S, 0)$ (see [17).

Remark 5.10. We define the lattice homomorphism $\phi_{j}: M_{j-1}^{\prime} \rightarrow M_{j},(\lambda, a) \mapsto \lambda+a\left(\lambda_{j}-\lambda_{j-1}\right)$. By Lemma 5.3 and induction we get that the restriction of the dual homomorphism $\phi_{j}^{*}: N_{j} \rightarrow$ $N_{j-1}^{\prime}$ defines an isomorphism of semigroups

$$
\phi_{j \mid \rho \cap N_{j}}^{*}: \rho \cap N_{j} \rightarrow \bar{\rho}_{j} \cap N_{j-1}^{\prime},
$$

for $j=1, \ldots, g$. This isomorphism induces a glueing of the cones $\bar{\rho}_{j} \in \Sigma_{j}$ and $\bar{\rho}_{j-1} \in \Sigma_{j-1}$, which preserves the lattice points. As a consequence of this isomorphism we deduce that the germ $\left(Z_{j}, o_{j}\right)$ is analytically isomorphic to the germ of normal toric variety $\left(Z_{\rho^{\prime}, N_{j}^{\prime}}, 0\right)$.

Remark 5.11. Glueing the fans $\Sigma_{j}, j=1, \ldots, g+1$ along the identifications (13), defines a conic polyhedral complex with integral structure $\bar{\Theta}$ associated to the q.o. hypersurface $(S, 0)$ (see [17]).

The theory of toroidal embeddings, introduced by Kempf et al. in [23] Chapter II, is a generalization of the theory of toric varieties. A toroidal embedding is a pair $(V, U)$, where $V$ is a normal variety and $U$ is an open smooth subvariety. In addition, the local algebra of $V$ at any point $v \in V$ is formally analytically isomorphic to the local algebra of an affine toric variety $Z$ with torus $T$, in such a way that the ideal of $V \backslash U$ corresponds to the ideal of $Z \backslash T$. The toroidal embedding is called without self-intersection if the irreducible components of $V-U$ are normal. In that case the toroidal embedding can be attached to a conic polyhedral complex with integral structure, a collection $\Xi$ of abstract rational polyhedral cones with certain glueing conditions. Their cells are in one to one correspondence with the strata of the stratification of $V$ associated to $(V, U)$. A finite rational polyhedral subdivision $\Xi^{\prime}$ of $\Xi$ induces a modification $V^{\prime} \rightarrow V$. This modification defines an isomorphism $U^{\prime} \rightarrow U$ over $U$, for some open subset $U^{\prime} \subset V^{\prime}$, in such a way that the pair $\left(V^{\prime}, U^{\prime}\right)$ is also a toroidal embedding. In particular, if $\Xi^{\prime}$ is a regular subdivision of $\Xi$ then the map $V^{\prime} \rightarrow V$ is a resolution of singularities of $V$.

In the case studied here, the pair $\left(Z_{g}, U\right)$ consisting of the normal variety $Z_{g}$ and the complement $U \subset Z_{g}$ of the strict transform of the hypersurface $\left\{x_{1} \cdots x_{d} f_{0} \cdots f_{g}=0\right\} \subset \mathbf{C}^{d+1}$ by the modification $\Pi_{1} \circ \cdots \circ \Pi_{g}$, defines a toroidal embedding without self-intersection. Its conic polyhedral complex is $\bar{\Theta}$ and this complex is isomorphic, by an isomorphism which preserves the integral structure, to the fan $\Theta$ of Definition 2.20 (see [17). Figure 1 represents the projectivization of the conic polyhedral complex $\bar{\Theta}$ associated to a q.o. surface with two characteristic monomials. The letters $f_{j}$ (resp. $x_{i}$ ) label the vertices associated to the strict transforms of $f_{i}=0$ (resp. $x_{i}=0$ ) by the modification $\Pi_{1} \circ \Pi_{2}$.

Theorem 5.12. ([17, Theorem 1) The proper morphism $\Pi:=\Pi_{1} \circ \cdots \circ \Pi_{g}$ is an embedded normalization of $(S, 0) \subset\left(\mathbf{C}^{d+1}, 0\right)$. An embedded resolution of $(S, 0)$,

$$
\Pi^{\prime}: Z^{\prime} \rightarrow\left(\mathbf{C}^{d+1}, 0\right)
$$

is obtained by composition of $\Pi$ with the modification associated to a regular subdivision $\bar{\Theta}^{\prime}$ of the conic polyhedral complex $\bar{\Theta}$. 


\section{Contact of the arcs With the Sequence of Semi-roots}

We consider the irreducible q.o. polynomial $f \in \mathbf{C}\left\{x_{1}, \ldots, x_{d}\right\}[y]$ together with its semi-roots $f_{0}, \ldots, f_{g}=f$.

Notation 6.1. We set

$$
F:=\left(x_{1}, \ldots, x_{d}, f_{0}, \ldots, f_{g}\right)
$$

We denote by $(V, 0) \subset\left(\mathbf{C}^{d+1}, 0\right)$ the hypersurface germ defined by $x_{1} \cdots x_{d} f_{0} \cdots f_{g}=0$. We denote by $\mathcal{H}$ the set of $\operatorname{arcs} \mathcal{H}:=\mathcal{L}\left(\mathbf{C}^{d+1}\right)_{0} \backslash \mathcal{L}(V)_{0}$. If $\varphi \in \mathcal{H}$ then the vector

$$
\operatorname{ord}_{t}(F \circ \varphi):=\left(\operatorname{ord}_{t}\left(x_{1} \circ \varphi\right), \ldots, \operatorname{ord}_{t}\left(x_{d} \circ \varphi\right), \operatorname{ord}_{t}\left(f_{0} \circ \varphi\right), \ldots, \operatorname{ord}_{t}\left(f_{g} \circ \varphi\right)\right),
$$

belongs to $\mathbf{Z}_{>0}^{d+g+1}$.

In this section we deal with the problem of determining which are the vectors $k \in \mathbf{Z}^{d+g+1}$ such that $k=\operatorname{ord}_{t}(F \circ \varphi)$, for some $\varphi \in \mathcal{H}$. For this purpose it is useful to pass by a coordinate free approach to deal with arcs in toric varieties.

Description of arcs in toric varieties. Let $N$ be a lattice of rank $d$ and $\theta \subset N_{\mathbf{R}}$ be a $d$-dimensional rational strictly convex cone. We denote by $O$ the origin of the toric variety $Z_{\theta}$.

An $\operatorname{arc} \psi \in \mathcal{L}\left(T_{N}\right)$ defines a group homomorphism

$$
M \rightarrow \mathbf{C}[[t]]^{*}, \quad m \mapsto x^{m} \circ \psi,
$$

where $\mathbf{C}[[t]]^{*}$ denotes the group of units of $\mathbf{C}[[t]]$. Conversely, any group homomorphism (14) defines an arc in the torus $T_{N}$.

The $\operatorname{arc} \varphi \in \mathcal{L}\left(Z_{\theta}\right)$ has generic point in the torus $T_{N}$ if and only if $x^{m} \circ \varphi \neq 0$, for any $m \in M=N^{*}$.

Definition 6.2. The functions

$$
\operatorname{ord}(\varphi): M \rightarrow \mathbf{Z}, \quad m \mapsto \operatorname{ord}_{t}\left(x^{m} \circ \varphi\right), \text { and } \operatorname{ac}(\varphi): M \rightarrow \mathbf{C}^{*}, \quad m \mapsto \operatorname{ac}\left(x^{m} \circ \varphi\right)
$$

are group homomorphisms.

Remark 6.3. If $\varphi \in \mathcal{L}\left(Z_{\theta}\right)$ has generic point in the torus then we have:

(i) The vector $\operatorname{ord}(\varphi)$ belongs to $N$ and $\operatorname{ac}(\varphi)$ defines a closed point in the torus $T_{N}$.

(ii) The condition $\varphi(0)=O$ holds if and only if $\operatorname{ord}_{t}\left(x^{m} \circ \varphi\right)>0, \forall m \in \theta^{\vee} \cap M \backslash\{0\}$, that is, if and only if $\operatorname{ord}(\varphi) \in \stackrel{\circ}{\theta} \cap N$.

(iii) The map

$$
\mathrm{u}(\varphi): M \rightarrow \mathbf{C}[[t]]^{*}, \quad m \mapsto t^{-\operatorname{ord}_{t}(\varphi)(m)}\left(x^{m} \circ \varphi\right)
$$

is a group homomorphism, i.e., an arc in the torus $T_{N}$.

(iv) The homomorphisms $\operatorname{ord}_{t}(\varphi)$ and $\mathrm{u}(\varphi)$ determine the $\operatorname{arc} \varphi$.

Lemma 6.4. Let $\Sigma$ be a fan subdividing the cone $\theta$. We denote by $\pi_{\Sigma}: Z_{\Sigma} \rightarrow Z_{\theta}$ the toric modification defined by $\Sigma$. If $\varphi \in \mathcal{L}\left(Z_{\theta}\right)_{O}$ is an arc with generic point in the torus, there is a unique arc $\varphi^{\prime} \in \mathcal{L}\left(Z_{\Sigma}\right)$ such that $\pi_{\Sigma} \circ \varphi^{\prime}=\varphi$. The origin $\varphi^{\prime}(0)$ of the lifted arc $\varphi^{\prime}$ belongs to the orbit $\operatorname{orb}(\tau)$ of the unique cone $\tau \in \Sigma$ such that $\operatorname{ord}(\varphi) \in \stackrel{\tau}{\tau}$. Moreover we have that

$$
\operatorname{ord}(\varphi)=\operatorname{ord}\left(\varphi^{\prime}\right) \text { and } \operatorname{ac}(\varphi)=\operatorname{ac}\left(\varphi^{\prime}\right) .
$$

Proof. By the valuative criterion of properness there is a unique $\operatorname{arc} \varphi^{\prime} \in \mathcal{L}\left(Z_{\Sigma}\right)$ lifting $\varphi$. The map $\pi_{\Sigma}$ is a proper modification which is the identity on the torus, hence equality (15) holds. The statement on the origin of the arc follows analogously as Remark 6.3 (ii). 
The order of contact of arcs in $\mathcal{H}$ with the sequence $F$. Now we apply the above notions to the case of an $\operatorname{arc} \varphi \in \mathcal{H}$.

As in Section 5 we assume that $y=f_{0}$. If $\pi$ denotes the projection (1) we get that $\pi \circ \varphi$ is an arc in $\mathcal{L}\left(\mathbf{C}^{d}\right)_{0}$ with generic point in the torus $T_{N_{0}}$ of $\mathbf{C}^{d}=Z_{\rho, N_{0}}$. Setting $\nu:=\operatorname{ord}(\pi \circ \varphi) \in N_{0}$ we deduce that

$$
\operatorname{ord}(\varphi)=\left(\nu, \operatorname{ord}_{t}(y \circ \varphi)\right) \in N_{0} \times \mathbf{Z}=N_{0}^{\prime} \text { and } \operatorname{ac}(\varphi): M_{0}^{\prime} \rightarrow \mathbf{C}^{*},
$$

correspond to the group homomorphisms introduced in Definition 6.2. The coordinates of $\nu$ with respect to the basis $\epsilon_{1}, \ldots, \epsilon_{d}$ of $N_{0}$ are $\left(\operatorname{ord}_{t}\left(x_{1} \circ \varphi\right), \ldots, \operatorname{ord}_{t}\left(x_{d} \circ \varphi\right)\right)$ (see Notation 2.16).

The toric map $\Pi_{1}$ of $\mathbf{C}^{d+1}$ induced by $\Sigma_{1}$ (see Section 5) is a proper modification, which is the identity over the torus $T_{N_{0}^{\prime}}$. Since $\varphi$ has generic point in $T_{N_{0}^{\prime}}$, by the valuative criterion of properness there is a unique lifting $\varphi^{(1)} \in \mathcal{L}\left(Z_{1}\right)$ of $\varphi$, i.e., there exists a unique $\varphi^{(1)} \in \mathcal{L}\left(Z_{1}\right)$ such that $\varphi=\Pi_{1} \circ \varphi^{(1)}$. The vector $\operatorname{ord}(\varphi)$ belongs to $\bar{\sigma}^{+}$(resp. $\bar{\sigma}^{-}$) if and only if

$$
\left\langle\nu, \lambda_{1}\right\rangle \leq(\text { resp. } \geq) \operatorname{ord}_{t}(y \circ \varphi) .
$$

Proposition 6.5 characterizes the case when $\varphi^{(1)}(0)=o_{1}$.

Proposition 6.5. The following conditions are equivalent:

(i) $\varphi^{(1)}(0)=o_{1}$,

(ii) $\operatorname{ord}_{t}\left(y_{1} \circ \varphi^{(1)}\right)>0$,

(iii) $\operatorname{ord}(\varphi) \in \operatorname{int}\left(\bar{\rho}_{1}\right)$ and $\left(f_{1}\right)_{\mid \operatorname{ord} \varphi}(\operatorname{ac}(\varphi))=0$.

Proof. We show first the equivalence (i) $\Leftrightarrow$ (ii). If $\varphi^{(1)}(0)=o_{1}$ then we get $\operatorname{ord}_{t}\left(y_{1} \circ \varphi^{(1)}\right)>0$, since $y_{1}$ belongs to a system of generators of the maximal ideal defining the point $o_{1}$ at $Z_{1}$. The other generators are monomials of the form $x^{m}$ for $m \in \rho^{\vee} \cap M_{1}$ and $m \neq 0$. For those vectors $m$ we have that $\operatorname{ord}_{t}\left(x^{m} \circ \varphi\right)=\operatorname{ord}_{t}\left(x^{m} \circ \varphi^{(1)}\right)>0$ by (15) and Remark 6.3.

Now we deal with the equivalence (ii) $\Leftrightarrow$ (iii). The condition $\operatorname{ord}(\varphi) \in \operatorname{int}\left(\bar{\rho}_{1}\right)$ is equivalent to $\varphi^{(1)}(0) \in \operatorname{orb}\left(\bar{\rho}_{1}\right)$. If $\operatorname{ord}(\varphi) \in \operatorname{int}\left(\bar{\rho}_{1}\right)$ then we get that $\left(f_{1}\right)_{\mid \operatorname{ord}(\varphi)}=y^{n_{1}}-c_{\lambda_{1}}^{n_{1}} x^{\lambda_{1}}$. Since $y_{1}=\left(x^{-n_{1} \lambda_{1}} f_{1} \circ \Pi_{1}\right)_{\mid Z_{\bar{\rho}_{1}}}$ we deduce that the constant term of the series $y_{1} \circ \varphi^{(1)}$ is equal to

$$
\operatorname{ac}\left(x^{-n_{1} \lambda_{1}} \circ \varphi\right)\left(\left(f_{1}\right)_{\mid \operatorname{ord}(\varphi)}(\operatorname{ac}(\varphi)) .\right.
$$

This ends the proof of the equivalence between (ii) and (iii).

If $\varphi \in \mathcal{H}$ then the series $y_{1} \circ \varphi^{(1)}$ is nonzero. Hence $\varphi^{(1)}$ can be seen as an arc with generic point in the torus $T_{N_{1}^{\prime}}$, associated to the toric structure of the germ $\left(Z_{1}, o_{1}\right)$ (see Lemma 5.4). By the valuative criterion of properness there is a unique lifting $\varphi^{(2)} \in \mathcal{L}\left(Z_{2}\right)$ of $\varphi^{(1)}$ such that $\Pi_{2} \circ \varphi^{(2)}=\varphi^{(1)}$. Notice also that $y_{2} \circ \varphi^{(2)} \neq 0$ by (12) and the definition of $\mathcal{H}$. By iterating this argument, using that $\varphi \in \mathcal{H}$, there exists a unique lifting of $\varphi^{(i)} \in \mathcal{L}\left(Z_{j}\right)$ such that $\Pi_{1} \circ \cdots \circ \Pi_{j} \circ \varphi^{(i)}=\varphi$, for $i=1, \ldots, g$.

We get different notions of orders of the lifted arcs which depend on the various toric structures appearing at toric resolution.

Notation 6.6. We denote by $\operatorname{ord}^{(i)}\left(\varphi^{(i)}\right): M_{i}^{\prime} \rightarrow \mathbf{Z}$ and $\operatorname{ac}^{(i)}\left(\varphi^{(i)}\right): M_{i}^{\prime} \rightarrow \mathbf{C}^{*}$, the group homomorphisms introduced in Definition 6.2, with respect to the toric structure of the germ $\left(Z_{i}, o_{i}\right)$ (see Remark 5.10). By convenience, we denote also $\varphi$ by $\varphi^{(0)}$ and $\operatorname{ord}(\varphi)$, ac $(\varphi)$ in (16) by $\operatorname{ord}^{(0)}\left(\varphi^{(0)}\right), \operatorname{ac}^{(0)}\left(\varphi^{(0)}\right)$ respectively. Sometimes $y_{0}$ also denotes $f_{0}$.

Proposition 6.7. Suppose that $\varphi^{(i)}(0)=o_{i}$ for $i=1, \ldots, j-1$. The following conditions are equivalent:

(i) $\varphi^{(j)}(0)=o_{j}$,

(ii) $\operatorname{ord}_{t}\left(y_{j} \circ \varphi^{(j)}\right)>0$,

(iii) $\operatorname{ord}^{(j-1)}\left(\varphi^{(j-1)}\right) \in \bar{\circ}_{j}$ and $\left(f_{j}^{(j-1)}\right)_{\mid \operatorname{ord}^{(j-1)}\left(\varphi^{(j-1)}\right)}\left(\operatorname{ac}\left(\varphi^{(j-1)}\right)\right)=0$.

Proof. The proof follows by induction using the same arguments as in Proposition 6.5.

Notice that if $\operatorname{ord}_{t}\left(y_{j} \circ \varphi^{(j)}\right)>0$ then the inequalities $\operatorname{ord}_{t}\left(y_{i} \circ \varphi^{(i)}\right)>0$ hold for $0 \leq i<j$.

We introduce the depth of an $\operatorname{arc} \varphi \in \mathcal{H}$, with respect to the modification $\Pi$ of Theorem 5.12 
Definition 6.8. If $\operatorname{ord}_{t}\left(y_{g} \circ \varphi^{(g)}\right)>0$ we define $\operatorname{depth}(\varphi):=g+1$. Otherwise, there exists a unique $1 \leq j \leq g$ such that $\operatorname{ord}_{t}\left(y_{j} \circ \varphi^{(j)}\right)=0$ and $\operatorname{ord}_{t}\left(y_{j-1} \circ \varphi^{(j-1)}\right)>0$, and then we set $\operatorname{depth}(\varphi)=j$.

If $\varphi \in \mathcal{H}$ is an arc of depth $j$ then $\varphi^{(i)} \in \mathcal{L}\left(Z_{i}\right)$ is an arc with origin at $o_{i}$, for $i=0, \ldots, j-1$.

Proposition 6.9. If $\varphi \in \mathcal{H}$ is an arc of depth $j>1$ and if $\nu=\operatorname{ord}(\pi \circ \varphi)$ then we have $\operatorname{ord}_{t}\left(y_{0} \circ \varphi\right)=\left\langle\nu, \lambda_{1}\right\rangle \quad$ and $\operatorname{ord}_{t}\left(y_{i} \circ \varphi^{(i)}\right)=\left\langle\nu, \lambda_{i+1}-\lambda_{i}\right\rangle, \quad$ for $i=2, \ldots, j-1$.

Proof. This is consequence of Proposition 6.7 and the definitions.

Lemma 6.10. If $\varphi \in \mathcal{H}$ is an arc of depth $j \geq 1$ with $\nu=\operatorname{ord}(\pi \circ \varphi)$, then, we have that

$$
\operatorname{ord}^{(i)}\left(\varphi^{(i)}\right)=\left(\nu, \operatorname{ord}_{t}\left(y_{i} \circ \varphi^{(i)}\right)\right) \text { for } i=0, \ldots, j-1 .
$$

Proof The assertion follows by Proposition 6.9 and induction. We get $\nu \in N_{i}$ using that $N_{g} \subset \cdots \subset N_{0}$.

Theorem 6.11. We have the following equalities:

$$
\left\{\operatorname{ord}_{t}(F \circ \varphi) \mid \varphi \in \mathcal{H}\right\}=\bigsqcup_{j=0}^{g+1} \bigsqcup_{\theta \in \Theta_{j}} \stackrel{\circ}{\theta} \cap \mathbf{Z}^{d+g+1}
$$

and if $1 \leq j \leq g+1$,

$$
\left\{\operatorname{ord}_{t}(F \circ \varphi) \mid \varphi \in \mathcal{H} \text { and } \operatorname{depth}(\varphi)=j\right\}=\bigsqcup_{\theta \in \Theta_{j}} \stackrel{\circ}{\theta} \cap \mathbf{Z}^{d+g+1} .
$$

Proof. It is enough to prove the equality (18). We assume first that $j<g+1$. If $\varphi \in \mathcal{H}$ and $\operatorname{depth}(\varphi)=j<g+1$ then there is a unique cone $\bar{\theta} \in\left\{\bar{\rho}_{j}, \bar{\sigma}_{j}^{+}, \bar{\sigma}_{j}^{-}\right\} \subset \Sigma_{j}$ such that

$$
\operatorname{ord}^{(j-1)}\left(\varphi^{(j-1)}\right) \in \operatorname{int}(\bar{\theta}) \cap N_{j-1}^{\prime} .
$$

We prove first that $\operatorname{ord}_{t}(F \circ \varphi)$ belongs to the the cone $\theta \in\left\{\rho_{j}, \sigma_{j}^{+}, \sigma_{j}^{-}\right\}=\Theta_{j}$. Suppose that $\bar{\theta}=\bar{\rho}_{j}$. By Proposition 6.9 we have that ord ${ }^{(j-1)}\left(\varphi^{(j-1)}\right)=(\nu, r)$ with $r=\left\langle\nu, \lambda_{j}-\lambda_{j-1}\right\rangle$. By Formula (12) we get,

$$
f_{i} \circ \varphi=\left(f_{i} \circ \Pi_{1} \circ \cdots \circ \Pi_{i}\right)_{\mid Z_{\bar{\rho}_{i}}} \circ \varphi^{(i)}=\left(x^{n_{i} \gamma_{i}} \circ \varphi\right) \cdot\left(y_{i} \circ \varphi^{(i)}\right),
$$

for $i=1, \ldots, j-1$, hence, taking orders we obtain:

$$
\operatorname{ord}_{t}\left(f_{i} \circ \varphi\right)=\left\langle\nu, n_{i} \gamma_{i}\right\rangle+\left\langle\nu, \lambda_{i+1}-\lambda_{i}\right\rangle=\left\langle\nu, \gamma_{i+1}\right\rangle \text {. }
$$

For $i=j$ we get

$$
\operatorname{ord}_{t}\left(f_{j} \circ \varphi\right)=\left\langle\nu, n_{j} \gamma_{j}\right\rangle
$$

since $\operatorname{ord}_{t}\left(y_{j} \circ \varphi^{(j)}\right)=0$ by hypothesis. For $g \geq i>j$ we deduce the equality $\left(f_{i} \circ \Pi_{1} \circ \cdots \circ\right.$ $\left.\Pi_{j}\right)_{\mid Z_{\bar{\rho}_{i}}}=x^{n_{j} \cdots n_{i} \gamma_{j}} \cdot f_{i}^{(j)}$ from Proposition 6.9. By construction, if $\operatorname{ord}_{t}\left(y_{j} \circ \varphi^{(j)}\right)=0$ then it follows that $\operatorname{ord}_{t}\left(f_{i}^{(j)} \circ \varphi^{(j)}\right)=0$ and then

$$
\operatorname{ord}_{t}\left(f_{i} \circ \varphi\right)=n_{i} \cdots n_{j+1}\left\langle\nu, n_{j} \gamma_{j}\right\rangle \text {. }
$$

We have shown that $\operatorname{ord}_{t}(F \circ \varphi)$ is an integral point in the relative interior of the cone $\rho_{j} \in \Theta_{j}$.

Conversely, we prove that any point $k=\left(k_{1}, \ldots, k_{d+g+1}\right) \in \stackrel{\circ}{\rho}_{j} \cap \mathbf{Z}^{d+g+1}$ is of the form $k=$ $\operatorname{ord}_{t}(F \circ \varphi)$, for some arc $\varphi \in \mathcal{H}$. By definition of $\rho_{j}$ if $\nu:=\left(k_{1}, \ldots, k_{d}\right) \in \stackrel{\circ}{\rho} \cap N_{0}$ we have the equalities

and

$$
k_{d+i}=\left\langle\nu, \gamma_{i}\right\rangle, \text { for } i=1, \ldots j,
$$

$$
k_{d+j+l}=n_{j+1} \cdots n_{j+l-1}\left\langle\nu, n_{j} \gamma_{j}\right\rangle, \text { for } l=1, \ldots g-j+1 .
$$

Since the vectors $e_{1}, \ldots, e_{d}, \gamma_{1}, \ldots, \gamma_{j}$ span the lattice $M_{j}$, the formulas above show that $\nu$ belongs to $N_{j}$. We get also that $k_{d+j}-n_{j} k_{d+j-1}=\left\langle\nu, \lambda_{j}-\lambda_{j-1}\right\rangle>0$ and $\left(\nu, k_{d+j}-n_{j} k_{d+j-1}\right) \in$ $\bar{\rho}_{j} \cap N_{j-1}^{\prime}$. Then, any arc $\psi \in \mathcal{L}\left(Z_{j-1}\right)_{o_{j-1}}$ with generic point in the torus $T_{N_{j-1}^{\prime}}$ and such that

$$
\operatorname{ord}^{(j-1)}(\psi)=\left(\nu, k_{d+j}-n_{j} k_{d+j-1}\right)
$$


verifies that $\varphi:=\Pi_{1} \circ \cdots \circ \Pi_{j-1} \circ \psi$ belongs to $\mathcal{H}, \varphi^{(j-1)}=\psi$ and $\operatorname{ord}_{t}(F \circ \varphi)=k$.

Now we consider the case when $\operatorname{ord}^{(j-1)}\left(\varphi^{(j-1)}\right)=(\nu, r)$ belongs to the interior of $\bar{\sigma}_{j}^{ \pm}$. By the previous arguments we get

$$
\operatorname{ord}_{t}\left(f_{i} \circ \varphi\right)=\left\langle\nu, \gamma_{i+1}\right\rangle, \text { for } i=0, \ldots, j-2, \text { and } \operatorname{ord}_{t}\left(f_{j-1} \circ \varphi\right)=\left\langle\nu, n_{j-1} \gamma_{j-1}\right\rangle+r,
$$

where $r=\operatorname{ord}_{t}\left(y_{j-1} \circ \varphi^{(j-1)}\right)$. We deduce the formula

$$
\left\{\begin{array}{cl}
\left\langle\nu, n_{j-1} \gamma_{j-1}\right\rangle & <\operatorname{ord}_{t}\left(f_{j-1} \circ \varphi\right)<\left\langle\nu, \gamma_{j}\right\rangle \\
\left\langle\nu, \gamma_{j}\right\rangle & \text { if } \quad(\nu, r) \in \bar{\sigma}_{j}^{-}, \\
\text {if } \quad(\nu, r) \in \bar{\sigma}_{j}^{+} .
\end{array}\right.
$$

Notice also that

$$
\operatorname{ord}_{t}\left(f_{j+l-1} \circ \varphi\right)=n_{j} \cdots n_{j+l-1}\left\langle\nu, n_{j-1} \gamma_{j-1}\right\rangle+\operatorname{ord}_{t}\left(f_{j+l-1}^{(j-1)} \circ \varphi^{(j-1)}\right)
$$

where

$$
\operatorname{ord}_{t}\left(f_{j+l-1}^{(j-1)} \circ \varphi^{(j-1)}\right)= \begin{cases}n_{j} \cdots n_{j+l-1} r & \text { if } \quad(\nu, r) \in \bar{\sigma}_{j}^{-}, \\ n_{j} \cdots n_{j+l-1}\left\langle\nu, \lambda_{j}-\lambda_{j-1}\right\rangle & \text { if } \quad(\nu, r) \in \bar{\sigma}_{j}^{+}\end{cases}
$$

for $1 \leq l \leq g-j+1$. We obtain from the definitions and the formulas (21), (22), (23) and (24) that $\operatorname{ord}_{t}(F \circ \varphi)$ belongs to $\operatorname{int}\left(\sigma_{j}^{-}\right) \cap \mathbf{Z}^{d+g+1}\left(\right.$ resp. $\left.\operatorname{int}\left(\sigma_{j}^{+}\right) \cap \mathbf{Z}^{d+g+1}\right)$ if $(\nu, r) \in \bar{\sigma}_{j}^{-}$(resp. if $\left.(\nu, r) \in \bar{\sigma}_{j}^{+}\right)$. We prove similarly that all the points in the sets $\operatorname{int}\left(\sigma_{j}^{ \pm}\right) \cap \mathbf{Z}^{d+g+1}$ are of this form.

The proof in the case of $j=g+1$ and $\bar{\theta}=\sigma_{g+1}$ is analogous.

We end this section with some auxiliary results.

Lemma 6.12. The map $s_{j}: \rho \cap N_{j} \mapsto \mathbf{Z}^{d+g+1}$ given by

$$
\nu \mapsto \nu+\sum_{i=1}^{j}\left\langle\nu, \gamma_{i}\right\rangle \epsilon_{d+i}+\sum_{i=j+1}^{g+1} n_{j} \cdots n_{i-1}\left\langle\nu, \gamma_{j}\right\rangle \epsilon_{d+i}
$$

defines an isomorphism of semigroups $\rho \cap N_{j} \cong \rho_{j} \cap \mathbf{Z}^{d+g+1}$.

Proof. This is consequence of the proof of Theorem 6.11.

Lemma 6.13. We fix an integer $1 \leq j \leq g$. The linear map $\Upsilon_{j}:\left(N_{j-1}^{\prime}\right)_{\mathbf{R}} \rightarrow \mathbf{R}^{d+g+1}$ given by

$$
(\nu, r) \mapsto \nu+\sum_{i=1}^{j-1}\left\langle\nu, \gamma_{i}\right\rangle \epsilon_{d+i}+\left(r+n_{j-1}\left\langle\nu, \gamma_{j-1}\right\rangle\right) \epsilon_{d+j}+\sum_{i=j+1}^{g+1} n_{j} \cdots n_{i-1}\left(r+n_{j-1}\left\langle\nu, \gamma_{j-1}\right\rangle\right) \epsilon_{d+i}
$$

verifies that $\Upsilon_{j}\left(\bar{\sigma}_{j}^{-} \cap N_{j-1}^{\prime}\right)=\sigma_{j}^{-} \cap \mathbf{Z}^{d+g+1}$ and $\Upsilon_{j}(0,1)=\epsilon_{d+j}+\sum_{i=j+1}^{g+1} n_{j} \cdots n_{i-1} \epsilon_{d+i}$. The restriction of $\Upsilon_{j}$ to $N_{j-1}^{\prime}$ is an isomorphism onto the lattice spanned by $\bar{\sigma}_{j}^{-} \cap \mathbf{Z}^{d+g+1}$. We set $\hat{\theta}:=\Upsilon_{j}(\theta)$ for $\theta \subset \rho^{\prime}$ a cone in $N_{j-1}^{\prime}$. The generating function of the set $\operatorname{int}(\hat{\theta}) \cap \mathbf{Z}^{d+g+1}$ is of the form $G_{\hat{\theta}}=P_{\hat{\theta}} \prod_{v}\left(1-x^{v}\right)$ (see Lemma 3.1). We denote by $\tilde{P}_{\hat{\theta}}$ the image of the polynomial $P_{\hat{\theta}}$ by the monomial map (5) and by $S_{\hat{\theta}}$ the rational function defined by replacing $\theta$ by $\hat{\theta}$ in Formula (6). The following equality holds:

$$
S_{\sigma_{j}^{-}}=S_{\hat{\rho}^{\prime}}-S_{\hat{\bar{\rho}}_{j}}-S_{\hat{\bar{\sigma}}_{j}^{+}}
$$

Proof. The assertion about the map $\Upsilon_{j}$ is consequence of the proof of Theorem 6.11. The formula (25) holds because $S_{\sigma_{j}^{-}}=S_{\hat{\bar{\sigma}}_{j}^{-}}$and $G_{\hat{\rho}^{\prime}}=G_{\hat{\bar{\rho}}_{j}}+G_{\hat{\bar{\sigma}}_{j}^{+}}+G_{\hat{\bar{\sigma}}_{j}^{-}}$by additivity of generating functions of integral points with respect to the partition $\operatorname{int}\left(\hat{\rho}^{\prime}\right)=\operatorname{int}\left(\hat{\bar{\rho}}_{j}\right) \sqcup \operatorname{int}\left(\hat{\bar{\sigma}}_{j}^{+}\right) \sqcup \operatorname{int}\left(\hat{\bar{\sigma}}_{j}^{-}\right)$.

\section{Computation of the motivic measure of $H_{k, 1}$}

In this section we compute the motivic measure of the set

$$
H_{k, 1}:=\left\{\varphi \in \mathcal{H} \mid \operatorname{ord}_{t}(F \circ \varphi)=k, \operatorname{ac}(f \circ \varphi)=1\right\} .
$$

by using the change of variables formula for motivic integrals (see Theorem [1.3). We assume in this section that $k$ is fixed and that $H_{k}$ is non-empty, hence by Theorem 6.11, there exists a unique $j \in\{1, \ldots, g+1\}$ and a unique cone $\theta \in \Theta_{j}$ such that vector $k$ belongs to $\stackrel{\circ}{\theta} \cap \mathbf{Z}^{d+g+1}$. 


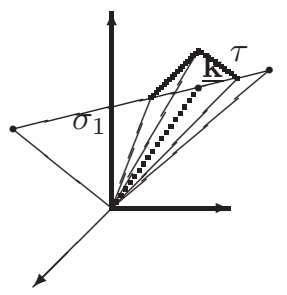

FiguRE 2.

We fix a regular subdivision $\bar{\Theta}^{\prime}$ of the conic polyhedral complex $\bar{\Theta}$ associated to the toric resolution of singularities, that is, a sequence $\Sigma_{i}^{\prime}$ of regular subdivisions of the fans $\Sigma_{i}$, which are compatible with the glueing of faces mentioned in Remark 5.10. By Proposition 6.9 the vector ord $^{(i-1)}\left(\varphi^{(i-1)}\right)$ belongs to $\bar{\rho}_{i}$, for $i=0, \ldots, j-1$ (see Definition 6.6). Let $\tau_{i} \in \Sigma_{i}^{\prime}$ be a $d+1$-dimensional regular cone containing the vector $\operatorname{ord}^{(i-1)}\left(\varphi^{(i-1)}\right)$. We can always chose a regular subdivision $\bar{\Theta}^{\prime}$ such that

$$
\operatorname{dim} \tau_{i} \cap \bar{\rho}_{i}=d-1, \text { for } i=1, \ldots, j-1, \quad \text { (see Figure 2). }
$$

The cone $\tau_{i}$ is generated by a basis $a_{1}^{(i)}, \ldots, a_{d+1}^{(i)}$ of the lattice $N_{i-1}^{\prime}$ and by the assumption (26) we can guarantee that $a_{1}^{(i)}, \ldots, a_{d}^{(i)} \in \bar{\rho}_{i}$. By Lemma 5.3 and Remark 5.10 the pair consisting of the cone $\bar{\rho}_{i}$ and the lattice generated by $\bar{\rho}_{i} \cap N_{i-1}^{\prime}$ is isomorphic to $\left(\rho, N_{i}\right)$. We identify these pairs under this isomorphism and we denote by $\mathcal{B}_{i}$ the basis of $N_{i}^{\prime}$ given by $\mathcal{B}_{i}:=\left\{\left(a_{1}^{(i)}, 0\right), \ldots,\left(a_{d}^{(i)}, 0\right),(0,1)\right\}$ for $i=1, \ldots, j-1$. We set $\mathcal{B}_{0}:=\left\{\epsilon_{1}, \ldots, \epsilon_{d+1}\right\}$ the canonical basis of $\mathbf{Z}^{d+1}$.

We denote by $x_{1}^{(i)}, \ldots, x_{d+1}^{(i)}$ the coordinates of $Z_{\tau_{i}, N_{i-1}^{\prime}} \cong \mathbf{C}^{d+1}$ associated to the dual basis of $a_{1}^{(i)}, \ldots, a_{d+1}^{(i)}$. The toric morphism $\pi_{\tau_{i}}$ is written in this coordinates as:

$$
\begin{array}{llllll}
x_{1}^{(i-1)} & = & \left(x_{1}^{(i)}\right)^{a_{1,1}^{(i)}} & \left(x_{2}^{(i)}\right)^{a_{2,1}^{(i)}} & \cdots & \left(x_{d+1}^{(i)}\right)^{a_{d+1,1}^{(i)}} \\
\cdots & \cdots & \cdots & \cdots & \cdots & \cdots \\
x_{d}^{(i-1)} & = & \left(x_{1}^{(i)}\right)^{a_{1, d}^{(i)}} & \left(x_{2}^{(i)}\right)^{a_{2, d}^{(i)}} & \cdots & \left(x_{d+1}^{(i)}\right)^{a_{d+1, d}^{(i)}} \\
y_{i-1} & = & \left(x_{1}^{(i)}\right)^{a_{1, d+1}^{(i)}} & \left(x_{2}^{(i)}\right)^{a_{2, d+1}^{(i)}} & \cdots & \left(x_{d+1}^{(i)}\right)^{a_{d+1, d+1}^{(i)}}
\end{array}
$$

where $\left(a_{r, 1}^{(i)}, \ldots, a_{r, d+1}^{(i)}\right)$ denote the coordinates of the vector $a_{r}^{(i)}$, for $r=1, \ldots, d+1$, with respect to the basis $\mathcal{B}_{i-1}$ of $N_{i-1}^{\prime}$.

We denote by $\mathbf{1}^{(i)}$ the vector in $M_{i}^{\prime}$ corresponding to the monomial $x_{1}^{(i)} \cdots x_{d}^{(i)} y_{i}$, for $i=$ $0, \ldots, j-1$.

Remark 7.1. We abuse of notation and denote by the same letter the lifting $\varphi^{(i)}$ of $\varphi$ by the maps $\pi_{\tau_{1}} \circ \cdots \circ \pi_{\tau_{i}}$ and $\Pi_{1} \circ \cdots \circ \Pi_{i}$. This causes no confusion since our analysis depends only on the group homomorphisms ord ${ }^{(i)}\left(\varphi^{(i)}\right)$ and $\mathrm{ac}^{(i)}\left(\varphi^{(i)}\right)$ of Notation 6.6. These homomorphisms coincide with those defined after taking the regular subdivisions by Lemma 6.4.

Proposition 7.2. With the previous notations if $\varphi \in H_{k}$ is of depth $j$ then

$$
\operatorname{ord}_{t}\left(\operatorname{Jac}\left(\pi_{\tau_{1}} \circ \cdots \circ \pi_{\tau_{j-1}}\right) \circ \varphi^{(j-1)}\right)=\xi_{j}(k)-\left\langle\operatorname{ord}^{(j-1)}\left(\varphi^{(j-1)}\right), \mathbf{1}^{(j-1)}\right\rangle .
$$

Proof. We have that the critical locus of the morphism $\pi_{\tau_{1}} \circ \cdots \circ \pi_{\tau_{j-1}}$ is defined by

$$
\operatorname{Jac}\left(\pi_{\tau_{1}} \circ \cdots \circ \pi_{\tau_{j-1}}\right)=\left(\operatorname{Jac}\left(\pi_{\tau_{1}}\right) \circ \pi_{\tau_{2}} \circ \cdots \circ \pi_{\tau_{j-1}}\right) \cdots\left(\operatorname{Jac}\left(\pi_{\tau_{j-1}}\right)\right) .
$$

From the expression (27) we get that

$$
\left.\operatorname{Jac}\left(\pi_{\tau_{i}}\right)\right)=\left(\left(x_{1}^{(i-1)} \cdots x_{d}^{(i-1)} y_{i-1}\right) \circ \pi_{\tau_{i}}\right)\left(x_{1}^{(i)} \cdots x_{d+1}^{(i)}\right)^{-1} .
$$

If $\varphi \in H_{k}$ our hipothesis guarantee that $\operatorname{ord}_{t}\left(x_{d+1}^{(i)} \circ \varphi^{(i)}\right)=0$, since $a_{d+1}^{(i)} \notin \bar{\rho}_{i}$. Composing with $\varphi^{(i)}$ at both sides of (29) and taking orders provides the equality:

$$
\operatorname{ord}_{t}\left(\operatorname{Jac}\left(\pi_{\tau_{i}}\right) \circ \varphi^{(i)}\right)=\left\langle\operatorname{ord}^{(i-1)} \varphi^{(i-1)}, \mathbf{1}^{(i-1)}\right\rangle-\left\langle\operatorname{ord}^{(i)} \varphi^{(i)}, \mathbf{1}^{(i)}\right\rangle+\operatorname{ord}_{t}\left(y_{i} \circ \varphi^{(i)}\right) .
$$


By definition of the lifting by the toric morphisms we get that $\pi_{\tau_{i+1}} \circ \cdots \circ \pi_{\tau_{j-1}} \circ \varphi^{(j-1)}=\varphi^{(i)}$, for any $0 \leq i<j-1$. We apply this observation when we take orders after composing both sides of (28) with $\varphi^{(j-1)}$. By using (30) we get that $\operatorname{ord}_{t}\left(\operatorname{Jac}\left(\pi_{\tau_{1}} \circ \cdots \circ \pi_{\tau_{j-1}}\right) \circ \varphi^{(j-1)}\right)$ is equal to

$$
\left\langle\operatorname{ord}^{(0)}(\varphi), \mathbf{1}^{(0)}\right\rangle-\left\langle\operatorname{ord}^{(j-1)} \varphi^{(j-1)}, \mathbf{1}^{(j-1)}\right\rangle+\sum_{i=1}^{j-1} \operatorname{ord}_{t}\left(y_{i} \circ \varphi^{(i)}\right) .
$$

By Proposition 6.9 and equality (19) we deduce that $\operatorname{ord}_{t}\left(y_{i} \circ \varphi^{(i)}\right)=k_{d+i+1}-n_{i} k_{d+i}$. The assertion follows since $\left\langle\operatorname{ord}^{(0)}(\varphi), \mathbf{1}^{(0)}\right\rangle=k_{1}+\cdots+k_{d+1}$. See Definition 2.21 .

Remark 7.3. If $\phi$ is a regular function on a smooth algebraic variety $Z$ and $E$ is an irreducible divisor on $E$ then the order of vanishing of $\phi$ along $E$ coincides with the order of $\phi \circ \alpha$, where $\alpha$ is a curvette at $E$, that is, $\alpha(0) \in E$ and $\alpha$ is a smooth arc transversal to $E$.

Remark 7.4. With the notations and hypothesis of Proposition 7.2 assume in addition that $\operatorname{ord}^{(j-1)}\left(\varphi^{(j-1)}\right)$ is one element of the basis $a_{1}^{(j-1)}, \ldots, a_{d+1}^{(j-1)}$. Then we deduce that

$$
\left.\operatorname{ord}_{t}\left(\operatorname{Jac}\left(\pi_{\tau_{1}} \circ \cdots \circ \pi_{\tau_{j-1}}\right)\right) \circ \varphi^{(j-1)}\right)=\xi_{j}(k)-1 .
$$

Notice that such a vector, say ord ${ }^{(j-1)}\left(\varphi^{(j-1)}\right)=a_{1}^{(j-1)}$, corresponds to an irreducible divisor $E$, which is defined on the chart $Z_{\tau_{j-1}, N_{j-1}^{\prime}}$ by $x_{1}^{(j-1)}=0$. By Lemma 6.4 there is a unique lifting $\varphi^{(j)}$ of the $\operatorname{arc} \varphi^{(j-1)}$ such that $\pi_{\tau_{j-1}} \circ \varphi^{(j)}=\varphi^{(j-1)}$ and $\varphi^{(j)}$ is a curvette at $E$. We abuse of notation and denote also by $E$ the strict transform of the divisor $E$ in $Z^{\prime}$, where we recall that $\Pi^{\prime}: Z^{\prime} \rightarrow \mathbf{C}^{d+1}$ is the toric embedded resolution of $(S, 0) \subset\left(\mathbf{C}^{d+1}, 0\right)$ (see Theorem 5.12). The order of vanishing of $\operatorname{Jac}\left(\Pi^{\prime}\right)$ (resp of $\Pi^{\prime} \circ f$ ) along the divisor $E$ is equal to $\xi_{j}(k)-1$ (resp $\eta(k))$. This follows from Formula (32) and Theorem 6.11 by using that these orders of vanishing along $E$ coincide with that of $\operatorname{Jac}\left(\pi_{\tau_{1}} \circ \cdots \circ \pi_{\tau_{j-1}}\right)$ (resp. of $\left.f \circ \pi_{\tau_{1}} \circ \cdots \circ \pi_{\tau_{j-1}}\right)$.

Remark 7.5. We apply remark 7.4 when $k=\nu_{i}^{(j)}$ for some $1 \leq i \leq d$ and $1 \leq j \leq g$, and then $E_{i}^{(j)}$ denotes the divisor $E$. See Remark 3.19 .

Recall the notations introduced in Definitions 2.13 and 3.6

Proposition 7.6. If $k \in \stackrel{\circ}{\theta} \cap \mathbf{Z}^{d+g+1}$ for some $\theta \in \Theta_{j}, j=1, \ldots g+1$, we have the following formula for the motivic measure of the set $H_{k, 1}$ :

$$
\mu\left(H_{k, 1}\right)=c_{1}(\theta)(\mathbf{L}-1)^{\operatorname{dim} \theta-1} \mathbf{L}^{-\xi_{j}(k)} .
$$

Proof. We deal first with the case when the arcs in $H_{k}$ are of depth one. The set $H_{k, 1}$ is stable for $l \geq \max \left\{k_{1}, \ldots, k_{d+1}\right\}$, thus the motivic measure of $\mu\left(H_{k, 1}\right)$ is equal to

$$
\mu\left(H_{k, 1}\right)=\left[\pi_{l}\left(H_{k, 1}\right)\right] \mathbf{L}^{-l(d+1)} .
$$

Now we compute the class $\left[\pi_{l}\left(H_{k, 1}\right)\right] \in K_{0}^{\hat{\mu}}\left(\operatorname{Var}_{\mathbf{C}}\right)$. If $\varphi \in H_{k, 1}$ we have the expansions

$$
x_{i} \circ \varphi=\operatorname{ac}\left(x_{i} \circ \varphi\right) t^{k_{i}}+c_{i, 1} t^{k_{i}+1}+\cdots+c_{i, l-k_{i}} t^{l} \quad \bmod t^{l+1} .
$$

The coefficients $c_{i, 1}, \ldots, c_{i, l-k_{i}}$, for $i=1, \ldots, d+1$, are free and contribute to the class $\left[\pi_{l}\left(H_{k, 1}\right)\right]$ with the factor $\mathbf{L}^{l(d+1)-\sum_{i=1}^{d+1} k_{i}}$.

The angular coefficients $\operatorname{ac}\left(x_{i} \circ \varphi\right)$ determine the point $\operatorname{ac}(\varphi) \in T_{N_{0}}$. Since $\varphi \in H_{k, 1}$ is of depth one, the equality $\operatorname{ac}(f \circ \varphi)=f_{\mid \operatorname{ord}(\varphi)}(\operatorname{ac}(\varphi))$ holds. It follows that the angular coefficients contribute to the class $\left[\pi_{l}\left(H_{k, 1}\right)\right]$ with the factor $\left[\left\{\operatorname{ac}(\varphi) \in T_{N_{0}^{\prime}} \mid f_{\mid \operatorname{ord}(\varphi)}(\operatorname{ac}(\varphi))=1\right\}\right]$. The result in this case follows by formula (7) and Definition 3.6

Now we assume that the arcs in $H_{k}$ are of depth $1<j \leq g+1$. We set

$$
H_{k, 1}^{(j-1)}:=\left\{\varphi^{(j-1)} \mid \varphi \in H_{k, 1}\right\} .
$$

We apply the change of variables formula for motivic integrals to the transformation $\Psi:=$ $\pi_{\tau_{1}} \circ \cdots \circ \pi_{\tau_{j-1}}$ (see Theorem [1.3).

$$
\mu\left(H_{k, 1}\right)=\int_{\varphi \in H_{k, 1}} d \mu=\int_{\varphi^{(j-1)} \in H_{k, 1}^{(j-1)}} \mathbf{L}^{-\operatorname{ord}_{t}\left(\operatorname{Jac}(\Psi) \circ \varphi^{(j)}\right)} d \mu .
$$


By Proposition 7.2 we get that

$$
\mu\left(H_{k, 1}\right)=\mathbf{L}^{-\xi_{j}(k)+\left\langle\operatorname{ord}^{(j-1)} \varphi^{(j-1)}, \mathbf{1}^{(j-1)}\right\rangle} \mu\left(H_{k, 1}^{(j-1)}\right) .
$$

Then the set $H_{k, 1}^{(j-1)}$ is stable for

$$
l \geq \max \left\{\operatorname{ord}_{t}\left(x_{1}^{(j-1)} \circ \varphi^{(j-1)}\right), \ldots, \operatorname{ord}_{t}\left(x_{d}^{(j-1)} \circ \varphi^{(j-1)}\right), \operatorname{ord}_{t}\left(y_{j-1} \circ \varphi^{(j-1)}\right)\right\},
$$

where the right-hand side of this formula is independent of the choice of $\varphi \in H_{k, 1}$. It follows that the motivic measure of $\mu\left(H_{k, 1}^{(j-1)}\right)$ is equal to

$$
\mu\left(H_{k, 1}^{(j-1)}\right)=\left[\pi_{l}\left(H_{k, 1}^{(j-1)}\right)\right] \mathbf{L}^{-l(d+1)} .
$$

The non-angular coefficients of the the expansions of the $\operatorname{arc} \varphi^{(j-1)}$ with respect to the coordinates $x_{1}^{(j-1)}, \ldots, x_{d}^{(j-1)}, y_{j-1}$, contribute to the class $\left[\pi_{l}\left(H_{k, 1}^{(j-1)}\right)\right]$ with the factor

$$
\mathbf{L}^{l(d+1)-\left\langle\operatorname{ord}^{(j-1)} \varphi^{(j-1)}, \mathbf{1}^{(j-1)}\right\rangle .} .
$$

It remains to compute the contribution of the angular coefficients.

We assume first that $j \leq g$. By (12) we get that $\left(f \circ \Pi_{1} \circ \cdots \circ \Pi_{j-1}\right)_{\mid Z_{\bar{\rho}_{j-1}}}=x^{e_{j-2} \gamma_{j-1}} f^{(j-1)}$ where

$$
f^{(j-1)}=\left(y_{j-1}^{n_{j}}-c x^{n_{j}\left(\lambda_{j}-\lambda_{j-1}\right)}\right)^{e_{j}}+\cdots, \quad \text { and } \quad f_{j}^{(j-1)}=\left(y_{j-1}^{n_{j}}-c x^{n_{j}\left(\lambda_{j}-\lambda_{j-1}\right)}\right)+\cdots,
$$

for some $c \neq 0$ and where the other terms have exponents outside the compact edge of the Newton polyhedron. By Proposition 6.7 the vector ord ${ }^{(j-1)}\left(\varphi^{(j-1)}\right)$ belongs to $\bar{\rho}_{j}$ hence we deduce that $\left(f^{(j-1)}\right)_{\mid \operatorname{ord}^{(j-1)}\left(\varphi^{(j-1)}\right)}=\left(\left(f_{j}^{(j-1)}\right)_{\mid \operatorname{ord}^{(j-1)}\left(\varphi^{(j-1)}\right)}\right)^{e_{j}}$ and $\left(f_{j}^{(j-1)}\right)_{\mid \operatorname{ord}^{(j-1)}\left(\varphi^{(j-1)}\right)}=$ $y_{j-1}^{n_{j}}-c x^{n_{j}\left(\lambda_{j}-\lambda_{j-1}\right)}$.

Since $\varphi$ is of depth $j$ we get that $y_{j} \circ \varphi^{(j)}$ is a series with non zero constant term. By (12) we have that $\left(f \circ \Pi_{1} \circ \cdots \circ \Pi_{j-1}\right)_{\mid Z_{\bar{\rho}_{j-1}}}=x^{e_{j-2} \gamma_{j-1}} f^{(j-1)}$. By Proposition 6.5 we obtain the equality $\operatorname{ac}(f \circ \varphi)=\left(x^{e_{j-2} \gamma_{j-1}}\left(\left(f_{j}^{(j-1)}\right)_{\mid \operatorname{ord}^{(j-1)}\left(\varphi^{(j-1)}\right)}\right)^{e_{j}}\right)\left(\operatorname{ac}^{(j-1)}\left(\varphi^{(j-1)}\right)\right)$. It follows that angular coefficients contribute to the class $\left[\pi_{l}\left(H_{k, 1}^{(j-1)}\right)\right]$ with the factor defined by the class of

$$
\left\{\operatorname{ac}^{(j-1)}\left(\varphi^{(j-1)}\right) \in T_{N_{j-1}^{\prime}} \mid x^{e_{j-2} \gamma_{j-1}}\left(f^{(j-1)}\right)_{\mid \operatorname{ord}^{(j-1)}\left(\varphi^{(j-1)}\right)}\left(\operatorname{ac}^{(j-1)}\left(\varphi^{(j-1)}\right)\right)=1 .\right\}
$$

In Lemma 7.7 below we prove that this class is equal to $c_{1}(\theta)(\mathbf{L}-1)^{\operatorname{dim} \theta-1}$.

If $\varphi$ is of depth $g+1$ then we get that $\operatorname{ac}(f \circ \varphi)=\operatorname{ac}\left(\left(x^{e_{g-1} \gamma_{g}} y_{g}\right) \circ \varphi^{(g)}\right)$. In this case the contribution of the angular coefficients to the class $\left[\pi_{l}\left(H_{k, 1}^{(j-1)}\right)\right]$ is $(\mathbf{L}-1)^{d}$.

Summarizing this discussion, by (36) we obtain that

$$
\left[\pi_{l}\left(H_{k, 1}^{(j-1)}\right)\right]=c_{1}(\theta)(\mathbf{L}-1)^{\operatorname{dim} \theta-1} \mathbf{L}^{l(d+1)-\left\langle\operatorname{ord}^{(j-1)}\left(\varphi^{(j-1)}\right), \mathbf{1}^{(j-1)}\right\rangle},
$$

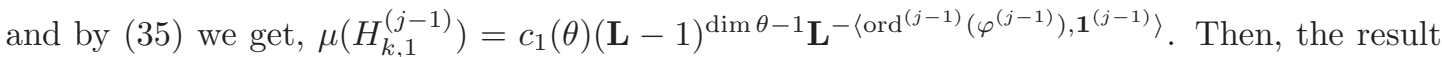
follows by (34) and (33).

Lemma 7.7. With hypothesis and notations of Proposition 7.6 and its proof, the class of (37) in $K_{0}^{\hat{\mu}}\left(\operatorname{Var}_{\mathbf{C}}\right)$ is equal to

$$
\begin{cases}{\left[\left\{(x, y) \in\left(\mathbf{C}^{*}\right)^{2} \mid\left(y^{n_{j}}-x^{r_{j}}\right)^{e_{j}}=1\right\}\right](\mathbf{L}-1)^{d-1}} & \text { if } k \in \rho_{j}, \\ {\left[\mu_{n_{j} e_{j}}\right](\mathbf{L}-1)^{d}} & \text { if } k \in \sigma_{j}^{-}, \\ {\left[\mu_{r_{j} e_{j}}\right](\mathbf{L}-1)^{d}} & \text { if } k \in \sigma_{j}^{+} .\end{cases}
$$

Proof. If $k \in \rho_{j}$ then (37) defines the hypersurface of $T_{N_{j-1}^{\prime}}=T_{N_{j-1}} \times \mathbf{C}^{*}$ with equation, $x^{e_{j-1} n_{j-1} \gamma_{j-1}}\left(y_{j-1}^{n_{j}}-c x^{n_{j}\left(\lambda_{j}-\lambda_{j-1}\right)}\right)^{e_{j}}=1$, for some $c \neq 0$. We have that $e_{j-2} \gamma_{j-1}=$ $e_{j} n_{j-1} n_{j} \gamma_{j-1}$, hence by (3) we obtain

$$
n_{j-1} n_{j} \gamma_{j-1}+n_{j}\left(\lambda_{j}-\lambda_{j-1}\right)=n_{j} \gamma_{j}
$$

We make first the change of coordinates in the torus defined by $y_{j-1}=x^{-n_{j-1} \gamma_{j-1}} u_{d+1}$. By (39) the set (37) is defined by the equation $\left(u_{d+1}^{n_{j}}-c x^{n_{j} \gamma_{j}}\right)^{e_{j}}=1$. By definition of $r_{j}$ there is a primitive vector $\tilde{\gamma}_{1} \in N_{j-1}$ such that $n_{j} \gamma_{j}=r_{j} \tilde{\gamma}_{1}$ (see Definition 2.13). We consider a basis of 
the lattice $N_{j-1}$ of the form $\tilde{\gamma}_{1}, \ldots, \tilde{\gamma}_{r}$. Setting $u_{i}:=x^{\tilde{\gamma}_{i}}$, for $i=1, \ldots, d$, defines coordinates $\left(u_{1}, \ldots, u_{d}\right)$ on the torus $T_{N_{j-1}}$. Then we get coordinates $\left(u_{1}, \ldots, u_{d+1}\right)$ on the torus $T_{N_{j-1}^{\prime}}$ such that the set (37) becomes

$$
\left\{u \in\left(\mathbf{C}^{*}\right)^{d+1} \mid\left(u_{d+1}^{n_{j}}-c u_{1}^{r_{j}}\right)^{e_{j}}=1 .\right\}
$$

This ends the proof in this case.

If $k \in \sigma_{j}^{-}$(resp. $k \in \sigma_{j}^{+}$) then (37) is the hypersurface of the torus $T_{N_{j-1}^{\prime}}$ defined by the equation, $x^{e_{j-2} \gamma_{j-1}} y_{j-1}^{n_{j} e_{j}}=1$, (resp. $\left.x^{e_{j} n_{j} \gamma_{j}}=1\right)$. In the coordinates $\left(u_{1}, \ldots, u_{d}, u_{d+1}\right)$ defined above (37) becomes $\left\{u \in\left(\mathbf{C}^{*}\right)^{d+1} \mid u_{d+1}^{n_{j} e_{j}}=1\right\}$ (resp. $\left\{u \in\left(\mathbf{C}^{*}\right)^{d+1} \mid u_{1}^{r_{j} e_{j}}=1\right\}$ ) and we are done.

\section{The PROOFS OF THE MAIN RESUltS}

We apply first the results of the previous sections to give a formula for the motivic zeta function of a q.o. polynomial in terms of the characteristic data. The following Proposition is crucial (see Definition 2.21).

Proposition 8.1. We have that $Z(f, T)_{0}=\sum_{j=1}^{g+1} \sum_{\theta \in \Theta_{j}} \sum_{k \in \Theta \cap \mathbf{Z}^{d+g+1}} \mu\left(H_{k, 1}\right) T^{\eta(k)}$.

Proof. The truncation map $\pi_{n}: \mathcal{L}\left(\mathbf{C}^{d+1}\right)_{0} \rightarrow \mathcal{L}_{n}\left(\mathbf{C}^{d+1}\right)_{0}$ is a surjection because $\mathbf{C}^{d+1}$ is smooth. Let us denote by $\mathcal{Z}_{n, 1} \subset \mathcal{L}\left(\mathbf{C}^{d+1}\right)_{0}$ the preimage of $\mathcal{X}_{n, 1}$ under $\pi_{n}$. The sets $\mathcal{Z}_{n, 1}$ are stable at level $n$ and thus $\mu\left(\mathcal{Z}_{n, 1}\right)=\left[\pi_{n}\left(\mathcal{Z}_{n, 1}\right)\right] \mathbf{L}^{-n(d+1)}=\left[\mathcal{X}_{n, 1}\right] \mathbf{L}^{-n(d+1)}$. We deduce that $Z(f, T)_{0}=\sum_{n} \mu\left(\mathcal{Z}_{n, 1}\right) T^{n}$.

For any integer $n \geq 0$ the sets $\mathcal{Z}_{n, 1}$ and $\mathcal{Z}_{n, 1} \cap \mathcal{H}$ have the same motivic measure since their difference is a subset of arcs contained in a hypersurface (see Lemma 1.2). By Theorem 6.11 we have the partition

$$
\mathcal{Z}_{n, 1} \cap \mathcal{H}=\bigsqcup_{j=1}^{g+1} \bigsqcup_{\theta \in \Theta_{j}} \bigsqcup_{\substack{\circ \\ k \in \theta \cap \mathbf{Z}^{d+g+1}}}^{\eta(k)=n} H_{k, 1}
$$

This partition may be non-finite. The result follows by using the properties of the motivic measure (see Proposition 1.2).

Lemma 8.2. We have that $\lim _{T \rightarrow \infty}(\mathbf{L}-1)^{\operatorname{dim} \theta-1} S_{\theta}=(-1)^{\ell_{g}+1}(\mathbf{L}-1)^{\ell_{g}}$ if $\theta=\sigma_{g+1}$. If $1 \leq j \leq g$ we get that:

$$
\lim _{T \rightarrow \infty}(\mathbf{L}-1)^{\operatorname{dim} \theta-1} S_{\theta}= \begin{cases}(-1)^{\ell_{j}}(\mathbf{L}-1)^{\ell_{j}-1} & \text { if } \theta=\sigma_{j}^{+}, \\ (-1)^{\ell_{j}}(\mathbf{L}-1)^{\ell_{j}-1} & \text { if } \theta=\rho_{j}, \\ (-1)^{\ell_{j-1}+1}(\mathbf{L}-1)^{\ell_{j-1}} & \text { if } \theta=\sigma_{j}^{-}\end{cases}
$$

Proof. The functions $S_{\theta}$ from Theorem 3.7 are rational functions in $\mathcal{M}_{\mathbf{C}}^{\hat{\mu}}[[T]]_{\text {sr }}$ of degree 0 with respect to $T$. If $1 \leq j \leq g$ the number of primitive vectors $v \in \mathbf{Z}^{d+g+1}$ generating an edge of $\rho_{j}$ such that $v_{d+g+1}=0$ is equal to the number $d-\ell_{j}$ of coordinates of $\lambda_{j}$ which are zero (see Lemma 2.22). In the case of the cone $\sigma_{j}^{+}$this number is equal to $d+1-\ell_{j}$ because we have to consider the edge spanned by $\epsilon_{d+j}$. Then we apply Lemma 3.2

In the case of the cone $\sigma_{j}^{-}$we use Lemma 6.13. It follows by Lemma 3.2 that $\lim _{T \rightarrow \infty}(\mathbf{L}-$ $1)^{d} S_{\hat{\rho}^{\prime}}=(-1)^{\ell_{j-1}+1}(\mathbf{L}-1)^{\ell_{j-1}}$ and $\lim _{T \rightarrow \infty}(\mathbf{L}-1)^{d} S_{\hat{\bar{\rho}}_{j}}=-\lim _{T \rightarrow \infty}(\mathbf{L}-1)^{d} S_{\hat{\bar{\sigma}}_{j}^{+}}$, since the last coordinate of the primitive vector $\Upsilon_{j}(0,1) \in \mathbf{Z}^{d+g+1}$ is nonzero. Then the assertion follows by equality (25).

We use the notations introduced in Section 3 .

Proof of Theorem 3.7. We deal first with the formula for the motivic zeta function. It is enough to compute the sum of the auxiliary series $R_{\theta}:=\sum_{k \in \dot{\theta} \cap \mathbf{Z}^{d+g+1}} \mu\left(H_{k, 1}\right) T^{\eta(k)}$, for $\theta \in \Theta_{j}$ and $1 \leq j \leq g+1$ (see Proposition 8.1). By Proposition [7.6 we get that $R_{\theta}=$ $c_{1}(\theta)(\mathbf{L}-1)^{\operatorname{dim} \theta-1} \sum_{k \in \Theta \cap \mathbf{Z}^{d+g+1}} \mathbf{L}^{-\xi_{j}(k)} T^{\eta(k)}$. By Lemma 3.1 and Notation 3.4 we deduce that $R_{\theta}=c_{1}(\theta)(\mathbf{L}-1)^{\operatorname{dim} \theta-1} S_{\theta}$, and then the conclusion follows. 
The formula for the motivic Milnor fiber appears now as a consequence of the formula for the motivic zeta function from Lemma 8.2 and Definition 3.6 .

We prove now the formula for the naive motivic zeta function. We consider the sets $H_{k}:=$ $\left\{\varphi \in \mathcal{H} \mid \operatorname{ord}_{t}(F \circ \varphi)=k\right\}$, for $k \in \mathbf{Z}_{\geq 0}^{d+g+1}$. By arguing as in Proposition 8.1 we get the formula

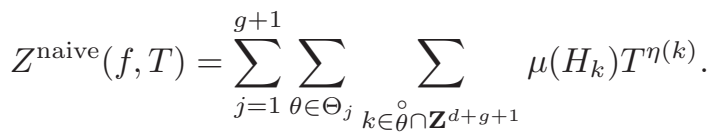

If $\theta \in \Theta_{j}$ the class of the set

$$
\left\{\operatorname{ac}^{(j-1)}\left(\varphi^{(j-1)}\right) \in T_{N_{j-1}^{\prime}} \mid x^{e_{j-2} \gamma_{j-1}}\left(f^{(j-1)}\right)_{\mid \operatorname{ord}^{(j-1)}\left(\varphi^{(j-1)}\right)}\left(\operatorname{ac}^{(j-1)}\left(\varphi^{(j-1)}\right)\right) \neq 0\right\}
$$

in $K_{0}\left(\operatorname{Var}_{\mathbf{C}}\right)$ equals

$$
\begin{cases}(\mathbf{L}-1)^{d+1} & \text { if } \quad k \in \sigma_{j}^{+}, \sigma_{j}^{-}, \sigma_{g+1} \\ (\mathbf{L}-1)^{d}(\mathbf{L}-2) & \text { if } \quad k \in \rho_{j} .\end{cases}
$$

This allows us to prove that the motivic measure of $H_{k}$ is $\mu\left(H_{k}\right)=c(\theta)(\mathbf{L}-1)^{\operatorname{dim} \theta-1} \mathbf{L}^{-\xi_{j}(k)}$ following the argument of Proposition 7.6 .

To prove the formula for the topological zeta function we need Proposition 1.11

If $1 \leq j \leq g+1$ and if $\theta \in \Theta_{j}$ is a $(d+1)$-dimensional simplicial cone, that is, $\theta=\sigma_{j}^{+}$if $j<g+1$ or $\theta=\sigma_{g+1}$ if $j=g+1$. By definition, using notations Lemma 3.1 and Definition 2.21. if $v_{1}, \ldots, v_{d+1} \in \mathbf{Z}^{d+g+1}$ are the primitive vectors generating the rays of $\theta$ we get the formula:

$$
c(\theta)(\mathbf{L}-1)^{\operatorname{dim} \theta-1} S_{\theta}=\left(\sum_{v \in D_{\theta}} \mathbf{L}^{-\xi(v)-s \eta(v)}\right) \prod_{i=1}^{d+1}(\mathbf{L}-1)\left(1-\mathbf{L}^{-\xi\left(v_{i}\right)-s \eta\left(v_{i}\right)}\right)^{-1}
$$

By applying $\chi_{\text {top }}$ to (41) provides the term $J_{\theta}(f, s)$ defined in 3.3 , since $\left[\mathbf{P}_{\mathbf{C}}^{a-1}\right]^{-1}=(\mathbf{L}-1)\left(\mathbf{L}^{a}-\right.$ 1) and $\chi_{\text {top }}\left(\left[\mathbf{P}_{\mathbf{C}}^{a-1}\right]\right)=a$. We deduce similarly that if $\theta=\rho_{j}$ for $1 \leq j \leq g$ then the result of applying $\chi_{\text {top }}$ to (41) is equal to $-J_{\rho_{j}}(f, s)$. The negative sign appears when applying $\chi_{\text {top }}$ to the term $(\mathbf{L}-2)$ of $c\left(\rho_{j}\right)$. Finally, we study the case of $\theta=\sigma_{j}^{-}$for $1 \leq j \leq g$, which may be not simplicial in general. We use Lemma 6.13. By (25) we have that

$$
c(\theta)(\mathbf{L}-1)^{\operatorname{dim} \theta-1} S_{\theta}\left(\mathbf{L}^{-s}\right)=(\mathbf{L}-1)^{d+1}\left(S_{\hat{\rho}^{\prime}}\left(\mathbf{L}^{-s}\right)-S_{\hat{\bar{\rho}}_{j}}\left(\mathbf{L}^{-s}\right)-S_{\hat{\bar{\sigma}}_{j}^{+}}\left(\mathbf{L}^{-s}\right)\right) .
$$

By Lemma 6.13 we get that $\operatorname{mult}\left(\hat{\rho}^{\prime}\right)=\operatorname{mult}\left(\rho_{j-1}\right), \operatorname{mult}\left(\hat{\bar{\sigma}}_{j}^{+}\right)=\operatorname{mult}\left(\sigma_{j}^{+}\right)$. By Lemma 6.13 we have the equalities $\xi_{j}\left(\Upsilon_{j}(0,1)\right)=1$ and $\eta\left(\Upsilon_{j}(0,1)\right)=n_{j} \ldots n_{g}$. Taking into account these observations we deduce that:

$$
\chi_{\text {top }}\left((\mathbf{L}-1)^{d+1} S_{\hat{\rho}^{\prime}}\right)\left(\mathbf{L}^{-s}\right)=\frac{J_{\rho_{j-1}}(f, s)}{1+n_{j} \cdots n_{g} s}, \quad \chi_{\text {top }}\left((\mathbf{L}-1)^{d+1} S_{\hat{\bar{\sigma}}_{j}^{+}}\left(\mathbf{L}^{-s}\right)\right)=\frac{\left.J_{\sigma_{j}^{+}}(f, s)\right)}{1+n_{j} \cdots n_{g} s},
$$

where the factor $\left(1+n_{j} \cdots n_{g} s\right)^{-1}$ corresponds to the vector $\Upsilon_{j}(0,1)$. Notice also that $\chi_{\text {top }}((\mathbf{L}-$ $\left.1)^{d+1} S_{\hat{\bar{\rho}}_{j}}\right)=0$. See Notation 3.3 .

Remark 8.3. We can expand $\chi_{\text {top }}\left((\mathbf{L}-1)^{d+1} S_{\sigma_{j}^{-}}\right)$in terms of a simplicial subdivision of the cone $\sigma_{j}^{-}$. Without loss of generality we assume in addition that the edges of the subdivision are exactly the edges of the cone $\sigma_{j}^{-}$. Such a subdivision always exists (see [13] Chapter 5, Theorem 1.2). If $\theta_{1}, \ldots, \theta_{l}$ are the $(d+1)$-dimensional cones in the subdivision we argue as before to obtain $\chi_{\text {top }}\left((\mathbf{L}-1)^{d+1} S_{\sigma_{j}^{-}}\right)=\sum_{i=1}^{l} \operatorname{mult}\left(\theta_{i}\right) \prod_{v_{i}}\left(\xi_{j}\left(v_{i}\right)+s \eta\left(v_{i}\right)\right)^{-1}$, where $v_{i} \in \mathbf{Z}^{d+g+1}$ runs through the primitive vectors generating the edges of $\theta_{i}$, for $i=1, \ldots, l$. Compare also with Lemma 5.1.1 [5].

\section{Comparison with the method of Newton maps}

The method of Newton maps allows to describe the parametrization of a q.o. hypersurface singularity. It has been used by Artal et al. 3] to study the poles of the motivic zeta function of a quasi-ordinary hypersurface singularity. We describe this method in geometric terms through the toric embedded resolution (see [17]). For simplicity in the exposition we restrict ourselves 
to the case of irreducible germs. We keep notations of the Sections 2, 4 and 5. As in Section 5] we assume that $y=f_{0}$ (see Definition 2.8).

Recall that we use the following notations: the canonical basis of $\mathbf{Z}^{d}=N_{0}$ is $\epsilon_{1}, \ldots, \epsilon_{d}$, the cone spanned by $\epsilon_{1}, \ldots, \epsilon_{d}$ is $\rho$, and $\varepsilon_{1}, \ldots, \varepsilon_{d}$ denotes the dual basis of the dual lattice $M_{0}$.

Definition 9.1. We define for $1 \leq j \leq g$ a sequence of vectors $\left\{\epsilon_{i}^{(j)}\right\}_{i=1}^{d}$ defining basis of a lattice $\tilde{N}_{j} \subset N_{0}$; then we denote by $\tilde{M}_{j} \supset M_{0}$ the dual lattice of $\tilde{N}_{j}$ equipped with the dual basis $\left\{\varepsilon_{i}^{(j)}\right\}_{i=1}^{d}$ of $\left\{\epsilon_{i}^{(j)}\right\}_{i=1}^{d}$. The definition is given in term of the characteristic exponents by induction on $j$. We expand $\lambda_{i}=\sum_{i=1}^{d} \frac{q_{i}^{(1)}}{p_{i}^{(1)}} \varepsilon_{i}$ where $\frac{q_{i}^{(1)}}{p_{i}^{(1)}}$ is an irreducible fraction for $i=1, \ldots, d$. Then we set

$$
\epsilon_{i}^{(1)}=p_{i}^{(1)} \epsilon_{i}, \text { for } i=1, \ldots, d .
$$

This defines the lattice $\tilde{N}_{1}$. With these notations we obtain

$$
\varepsilon_{i}=p_{i}^{(1)} \varepsilon_{i}^{(1)}, \text { for } i=1, \ldots, d \text {, and } \lambda_{1}=q_{1}^{(1)} \varepsilon_{1}^{(1)}+\cdots+q_{d}^{(1)} \varepsilon_{d}^{(1)},
$$

hence we get that $M_{1} \subset \tilde{M}_{1}$ and $\tilde{N}_{1} \subset N_{1}$. We suppose that $1<j \leq g$ and $\tilde{M}_{j-1}$ together with its basis $\left\{\varepsilon_{i}^{(j-1)}\right\}_{i=1}^{d}$ have being defined by induction. Then we expand the vector $\lambda_{j}-\lambda_{j-1}$ in the basis $\left\{\varepsilon_{i}^{(j-1)}\right\}_{i=1}^{d}$. We obtain $\lambda_{j}-\lambda_{j-1}=\sum_{i=1}^{d} \frac{q_{i}^{(j)}}{p_{i}^{(j)}} \varepsilon_{i}^{(j-1)}$, where $\frac{q_{i}^{(j)}}{p_{i}^{(j)}}$ are irreducible fractions. Then, with these notations we define the basis $\left\{\epsilon_{i}^{(j)}\right\}_{i=1}^{d}$ of the lattice $\tilde{N}_{j}$ by setting:

$$
\epsilon_{i}^{(j)}=p_{i}^{(j)} \epsilon_{i}^{(j-1)}, \text { for } i=1, \ldots, d
$$

We get that

$$
\varepsilon_{i}^{(j-1)}=p_{i}^{(j)} \varepsilon_{i}^{(j)}, \text { for } i=1, \ldots, d, \quad \lambda_{j}-\lambda_{j-1}=q_{1}^{(j)} \varepsilon_{1}^{(j)}+\cdots+q_{d}^{(j)} \varepsilon_{d}^{(j)},
$$

$M_{j} \subset \tilde{M}_{j}$ and $\tilde{N}_{j} \subset N_{j}$.

By definition the cone $\rho$ is regular for the lattice $\tilde{N}_{j}$. Hence the $d$-dimensional toric variety $Z_{\rho, \tilde{N}_{j}}$ is isomorphic to $\mathbf{C}^{d}$. We have a Z-bilinear pairing $N_{j} \times \tilde{M}_{j} \rightarrow \mathbf{Q},(\nu, \gamma) \mapsto\langle\nu, \gamma\rangle$ which extends the duality pairings $\tilde{N}_{j} \times \tilde{M}_{j} \rightarrow \mathbf{Z}$ and $N_{j} \times M_{j} \rightarrow \mathbf{Z}$. The inclusion of lattices $\tilde{N}_{j} \subset N_{j}$ induces a proper equivariant map

$$
\kappa_{j}: Z_{\rho, \tilde{N}_{j}} \longrightarrow Z_{\rho, N_{j}}
$$

which is the projection for the quotient of $Z_{\rho, \tilde{N}_{j}}$ with respect to the natural action of the finite abelian group $G_{j}:=N_{j} / \tilde{N}_{j}$. This action is defined on monomials and extended by linearity. If $\nu+\tilde{N}_{j} \in N_{j} / \tilde{N}_{j}$ and $\gamma \in \tilde{M}_{j}$ then the action is given by $\left(\nu+\tilde{N}_{j}\right) \cdot x^{\gamma}:=e^{2 \pi i\langle\nu, \gamma\rangle} x^{\gamma}$. The subset of invariant monomials by this action is $\left\{x^{\gamma} \mid \gamma \in M_{j}\right\}$. The restriction of the map (46) to the torus $T_{\tilde{N}_{j}} \rightarrow T_{N_{j}}$ is an unramified covering of degree $\left|G_{j}\right|$ and the kernel of this map is isomorphic to $G_{j}$. See [31, Corollary 1.16].

Lemma 9.2. The primitive integral vectors of the cone $\bar{\rho}_{j}$ with respect to the lattice $N_{j-1} \times \mathbf{Z}$ (and also with respect to the lattice $\tilde{N}_{j-1} \times \mathbf{Z}$ ) are $\left(p_{i}^{(j)} \epsilon_{i}^{(j-1)}, q_{i}^{(j)}\right)$ for $i=1, \ldots, d$.

Proof. In Lemma 5.3 and Remark 5.10 we introduced for $1 \leq j \leq g$ the lattice homomorphisms $\phi_{j}: M_{j-1}^{\prime} \rightarrow M_{j}$ in such a way that we can identify $M_{j}$ with the quotient lattice $M_{j-1}^{\prime} / \operatorname{ker}\left(\phi_{j}\right)$. The dual lattice homomorphism $\phi_{j}^{*}: N_{j} \rightarrow N_{j-1}^{\prime}$ is injective and by (45) it verifies that

$$
\phi_{j}^{*}\left(\epsilon_{i}^{(j)}\right)=\left(p_{i}^{(j)} \epsilon_{i}^{(j-1)}, q_{i}^{(j)}\right), \quad i=1, \ldots, d
$$

are the primitive integral vectors for the lattice $N_{j-1}^{\prime}$ (and also for $\tilde{N}_{j-1} \times \mathbf{Z}$ ) in the edges of the cone $\bar{\rho}_{j} \subset\left(N_{j-1}^{\prime}\right)_{\mathbf{R}}$.

Remark 9.3. By the arguments in Lemma 9.2 we can identify $N_{j}$ with its image $\operatorname{Im}\left(\phi_{j}^{*}\right)=$ $\left(\operatorname{ker}\left(\phi_{j}\right)\right)^{\perp}$, which is equal to the subset of $N_{j-1}^{\prime}$ in the linear hull of the cone $\bar{\rho}_{j}$. We deduce that $\phi_{j}^{*}(\rho)=\bar{\rho}_{j}$ hence the map $\phi_{j}^{*}$ defines an isomorphism of toric varieties $Z_{\rho, N_{j}} \rightarrow Z_{\bar{\rho}_{j}, \phi_{j}^{*}\left(N_{j}\right)}$. 
The following maps are defined in terms of the characteristic exponents for $j=1, \ldots, g$, with respect to suitable choices of coordinates.

Definition 9.4. The Newton map $\mathcal{N}_{j}: \mathbf{C}^{d} \times \mathbf{C}^{*} \rightarrow \mathbf{C}^{d+1}$ is the monomial map

$$
\left(x_{1}^{(j)}, \ldots, x_{d}^{(j)}, z_{j}\right) \rightarrow\left(x_{1}^{(j-1)}, \ldots, x_{d}^{(j-1)}, \tilde{y}_{j-1}\right)
$$

given by:

$$
\left\{\begin{array}{l}
x_{i}^{(j-1)}=\left(x_{i}^{(j)}\right)^{p_{i}^{(j)}}, \text { for } i=1, \ldots, d \\
\tilde{y}_{j-1}=\left(x_{1}^{(j)}\right)^{q_{1}^{(j)}} \cdots\left(x_{d}^{(j)}\right)^{q_{d}^{(j)}} z_{j}
\end{array}\right.
$$

We express the Newton map $\mathcal{N}_{j}$ in terms of the restriction of the map $\Pi_{j}$ to the chart $Z_{\bar{\rho}_{j}, N_{j-1}^{\prime}}$. We denote this restriction also by $\Pi_{j}$. By Lemma 5.3 and Remark 5.10 the coordinate ring of $Z_{\bar{\rho}_{j}, N_{j-1}^{\prime}}$ is isomorphic to $\mathbf{C}\left[\rho^{\vee} \cap M_{j}\right]\left[w_{j}^{ \pm 1}\right]$ and $Z_{\bar{\rho}_{j}, N_{j-1}^{\prime}}$ is equivariantly isomorphic to $Z_{\rho, N_{j}} \times \mathbf{C}^{*}$. Setting $w_{j}=z_{j}^{n_{j}}$ defines a map

$$
\psi_{j}: Z_{\rho, N_{j}} \times \mathbf{C}^{*} \rightarrow Z_{\rho, N_{j}} \times \mathbf{C}^{*},
$$

which is an unramified covering of degree $n_{j}$. This map also appears in [17] Section 3.2.1.

Proposition 9.5. The Newton map $\mathcal{N}_{1}$ is equal to the composite

$$
Z_{\rho, \tilde{N}_{1}} \times \mathbf{C}^{*} \stackrel{\kappa_{1} \times I d}{\longrightarrow} Z_{\rho, N_{1}} \times \mathbf{C}^{*} \stackrel{\psi_{1}}{\longrightarrow} Z_{\rho, N_{1}} \times \mathbf{C}^{*} \stackrel{\Pi_{1}}{\longrightarrow} \mathbf{C}^{d+1} .
$$

Proof. By using the formula (43) the relation $y=\left(x_{1}^{(1)}\right)^{q_{1}^{(1)}} \cdots\left(x_{d}^{(1)}\right)^{q_{d}^{(1)}} z_{1}$, can be rewritten as $z_{1}=y x^{-\lambda_{1}}$. Then apply that $w_{1}=z_{1}^{n_{1}}$. It follows that then composite map above is defined by the homomorphism of semigroups $\mathbf{Z}_{\geq 0}^{d+1} \rightarrow\left(\rho^{\vee} \cap \tilde{M}_{1}\right) \times \mathbf{Z}$ which maps $i$-th canonical basic vector of $\mathbf{Z}^{d+1}$ to $\left(p_{i}^{(1)} \varepsilon_{i}^{(1)}, 0\right)$ if $1 \leq i \leq d$ or to $\sum_{i=1}^{d} q_{i}^{(1)}\left(\varepsilon_{i}^{(1)}, 0\right)+(0,1)$ if $i=d+1$. Then the result follows from the definitions.

Remark 9.6. In the [3, Definition 3.20] the Newton map is defined by replacing $z_{1}$ in (48) by $z_{1}-\alpha_{1}$ for some suitable $\alpha_{1} \in \mathbf{C}^{*}$. This translation corresponds in our definition to the choice of a suitable point $o_{1}^{\prime}$ in the fiber $\psi_{1}^{-1}\left(o_{1}\right)$.

We deduce some consequences of Proposition 9.5 and the results stated in Section 5

We know that the strict transform $S^{(1)}$ of $S$ by the modification $\Pi_{1}$ is a germ at the point $o_{1} \in\{0\} \times \mathbf{C}^{*} \subset Z_{\rho, N_{1}} \times \mathbf{C}^{*}$. It follows that the fiber of $o_{1}$ by $\psi_{1}$ consists of $n_{1}$ different points. Let us fix one point $o_{1}^{\prime} \in \psi_{1}^{-1}\left(o_{1}\right)$. The map $\psi_{1}$ defines an isomorphism of germs $\left(Z_{\rho, N_{1}} \times \mathbf{C}^{*}, o_{1}^{\prime}\right) \rightarrow\left(Z_{\rho, N_{1}} \times \mathbf{C}^{*}, o_{1}\right)$. The preimage by $\kappa_{1} \times I d$ of the point $o_{1}^{\prime}$ has only one point which we denote by $\tilde{o}_{1}$. We get that the germ of the strict transform $\tilde{S}^{(1)}$ of $(S, 0)$ by the Newton map $\mathcal{N}_{1}$ at the point $\tilde{o}_{1}$ coincides with the germ at $\tilde{o}_{1}$ of the preimage of $\left(S^{(1)}, o_{1}\right)$ by the map $\psi_{1} \circ\left(\kappa_{1} \times I d\right)$. The germs defined in this way by picking different points in the fiber $\psi^{-1}\left(o_{1}\right)$ are isomorphic. Proposition 9.5 also implies that the germ $\left(\tilde{S}^{(1)}, \tilde{o}_{1}\right)$ is invariant by the action of the group $G_{1}$. The germ $\left(S^{1}, o_{1}\right)$ is a t.q.o. singularity (see Remark (5.6). We deduce that $\left(\tilde{S}^{(1)}, \tilde{o}_{1}\right)$ is a germ of q.o. hypersurface singularity. If $g=1$ then Proposition 9.5 implies that $\left(\tilde{S}^{(1)}, \tilde{o}_{1}\right)$ is smooth and the partial toric resolution procedure and the Newton map procedure end after one step.

Assume that $g>1$. We take a local coordinate $\tilde{y}_{1}$ at $\tilde{o}_{1}$, which defines the germ $\left(\tilde{S}_{1}^{(1)}, \tilde{o}_{1}\right)$, that is, the strict transform of the semi-root $\left(S_{1}, 0\right)$. Notice that $\left(\tilde{S}_{1}^{(1)}, \tilde{o}_{1}\right)$ is invariant by the action of $G_{1}$. Notice that the germ $\left(Z_{\rho, \tilde{N}_{1}} \times \mathbf{C}^{*}, \tilde{o}_{1}\right)$ is isomorphic to $\left(\mathbf{C}^{d+1}, 0\right)$ with coordinates $x_{1}^{(1)}, \ldots, x_{d}^{(1)}, \tilde{y}_{1}$. In Section 5 we proved also that the germ $\left(Z_{\rho, N_{1}} \times \mathbf{C}^{*}, o_{1}\right)$ is isomorphic to $\left(Z_{\rho, N_{1}} \times \mathbf{C}, 0\right)$ with the coordinates given by $y_{1}$ and $x^{\gamma}$ for $\gamma \in \rho^{\vee} \cap M_{1}$. From Proposition 9.5. we deduce that the Newton polyhedron of the series defining the strict transform $\left(\tilde{S}^{(1)}, \tilde{o}_{1}\right)$ with respect to the coordinates $x_{1}^{(1)}, \ldots, x_{d}^{(1)}, \tilde{y}_{1}$ coincides with the Newton polyhedron of the series $f^{(1)} \in \mathbf{C}\left\{\rho^{\vee} \cap M_{1}\right\}\left\{y_{1}\right\}$ defining the strict transform $\left(S^{(1)}, o_{1}\right)$. It follows that both polyhedra define the same dual subdivision $\Sigma_{2}$. By Lemma 9.2 the primitive integral vectors in edges of the cone $\bar{\rho}_{2} \in \Sigma_{2}$ are the same for the lattices $\tilde{N}_{1} \times \mathbf{Z}$ and $N_{1} \times \mathbf{Z}$. The Newton 
map $\mathcal{N}_{2}$ is defined in terms of these primitive vectors. We deduce, as in Proposition 9.5, that $\Pi_{2} \circ \psi_{2} \circ\left(\kappa_{2} \times I d\right)=\left(\kappa_{1} \times I d\right) \circ \mathcal{N}_{2}$. If $g>2$ we iterate this procedure as in Section 5 We can extend these notions to the level $1<j<g$. At the level $j-1$ we choose one point $\tilde{o}_{j-1}$ in the fiber $\left(\psi_{j-1} \circ\left(\kappa_{j-1} \times I d\right)\right)^{-1}\left(o_{j-1}\right)$. Then the Newton map $\mathcal{N}_{j}: Z_{\rho, \tilde{N}_{j}} \times \mathbf{C}^{*} \rightarrow Z_{\rho, \tilde{N}_{j-1}} \times \mathbf{C}$ can be also defined in terms of the Newton polyhedron of the strict transform of $f$ at the point $\tilde{o}_{j-1}$, with respect to the coordinates $x_{1}^{(j-1)}, \ldots, x_{d}^{(j-1)}, \tilde{y}_{j-1}$ of $Z_{\rho, \tilde{N}_{j-1}} \times \mathbf{C}$, where $\tilde{y}_{j-1}$ denotes the strict transform of the semiroot $f_{j-1}$.

The interplay between the Newton maps and the toric resolution is summarized by:

Proposition 9.7. With the notations above for $1 \leq j \leq g$ we have that

$$
\Pi_{j} \circ \psi_{j} \circ\left(\kappa_{j} \times I d\right)=\left(\kappa_{j-1} \times I d\right) \circ \mathcal{N}_{j} .
$$

The strict transform $\tilde{S}^{(j)}$ at the point $\tilde{o}_{j}$ of the q.o. hypersurface $(S, 0)$ by the iteration of the first $j$ Newton maps is invariant by the action of $G_{j}$. The image of the germ $\left(\tilde{S}^{(j)}, \tilde{o}_{j}\right)$ by the map $\left(\kappa_{j} \times I d\right) \circ \psi_{j}$ is equal to $\left(S^{(j)}, o_{j}\right)$ for $j=1, \ldots, g$. The germ $\left(\tilde{S}^{(g)}, \tilde{o}_{g}\right)$ is smooth and the map $\left(\tilde{S}^{(g)}, \tilde{o}_{g}\right) \rightarrow\left(S^{(g)}, o_{g}\right)$ is the canonical orbifold map associated to the normalization $\left(S^{(g)}, o_{g}\right)$ of the germ $(S, 0)$.

Remark 9.8. See 33 for a topological description of the canonical orbifold map.

Remark 9.9. The germ $\left(S^{(1)}, o_{1}\right)$ is irreducible however if $d \geq 2$ and $\left|G_{1}\right|>1$ the germ $\left(\tilde{S}^{(1)}, \tilde{o}_{1}\right)$ it is not analytically irreducible in general (see Example 10.1). It may happen that $\left(\tilde{S}^{(1)}, \tilde{o}_{1}\right)$ have irreducible factors which are not invariant by the action of $G_{1}$. This phenomenon of "false reducibility" has been analyzed and explained in a combinatorial way in [19.

Comparison of the sets of candidate poles. Artal et al. define in [3. Definition 3.28] a set of pairs $C P(f, \omega)$ where $f$ is a quasi-ordinary polynomial, $\omega$ is certain differential form and the coordinates are choosen in a suitable way. We assume here that $f \in \mathbf{C}\left\{x_{1}, \ldots, x_{d}\right\}[y]$ is an irreducible q.o. polynomial as in Section [5, $y=f_{0}$ and $\omega=d x_{1} \wedge \cdots \wedge d x_{d} \wedge d y$.

Definition 9.10. We define first $C P(f, \omega)$ as the list of pairs $\left\{\left(B_{i}^{(j)}, b_{i}^{(j)}\right)\right\}_{i=1, \ldots, d}^{j=1, \ldots, g} \cup\{(1,1)\}$, where for $1 \leq i \leq d$ and $1<j \leq g$ we set

$B_{i}^{(1)}:=e_{0} q_{i}^{(1)}, \quad B_{i}^{(j)}:=p_{i}^{(j)} B_{i}^{(j-1)}+e_{j-1} q_{i}^{(j)}$ and $b_{i}^{(1)}:=p_{i}^{(1)}+q_{i}^{(1)}, \quad b_{i}^{(j)}:=p_{i}^{(j)} b_{i}^{(j-1)}+q_{i}^{(j)}$.

The number $B_{i}^{(j)}$ (resp. $b_{i}^{(j)}-1$ ) is equal to the order of vanishing of the pull-back of $f$ (resp. of $\omega$ ) under the sequence of first $j$ Newton maps along the divisor $x_{i}^{(j)}=0$. These formulas follow easily by induction using the definition of the Newton maps (see [3]). The terms $1-\mathbf{L}^{-a} T^{A}$ for $(A, a) \in C P(f, \omega)$ form a set of candidate poles of the zeta function $Z^{\text {naive }}(f, T)$ as computed in 3 .

In order to compare with our results we consider the cone $\rho$ embedded in $\mathbf{R}^{d+g+1}=\mathbf{R}^{d} \times \mathbf{R}^{g+1}$ as $\rho_{0}=\rho \times\{0\}$ (see Notation 2.19). By Lemma 6.12 if $1 \leq i \leq d$ the vector $\nu_{i}^{(j)}:=s_{j}\left(\epsilon_{i}^{(j)}\right.$ ) is primitive for the lattice $\mathbf{Z}^{d+g+1}$ and defines an edge of the cone $\rho_{j}$.

Lemma 9.11. We have that $B_{i}^{(j)}=\eta\left(\nu_{i}^{(j)}\right)$ and $b_{i}^{(j)}=\xi_{j}\left(\nu_{i}^{(j)}\right)$ for $1 \leq i \leq d$ and $1 \leq j \leq g$.

Proof. We prove these equalities by induction on $j$. If $j=1$ they hold by the definitions. Assume that $j>1$ and that the result holds for $j-1$.

We deal first with the equality for $B_{i}^{(j)}$. We argue as in the proof of Theorem 6.11 The vector $\nu_{i}^{(j)}=\operatorname{ord}_{t} F \circ \varphi$ for some $\operatorname{arc} \varphi$ of depth $j$. By Lemma 9.2 and (44) we get $\eta\left(\nu_{i}^{(j)}\right)=e_{j-1}\left\langle\epsilon_{i}^{(j)}, \gamma_{j}\right\rangle$ and similarly $\eta\left(\nu_{i}^{(j)}\right)=e_{j-2}\left\langle\epsilon_{i}^{(j-1)}, \gamma_{j-1}\right\rangle$. Since $\epsilon_{i}^{(j)}=p_{i}^{(j)} \epsilon_{i}^{(j-1)}$ we deduce using (3) that

$$
\eta\left(\nu_{i}^{(j)}\right)=p_{i}^{(j)} e_{j-2}\left\langle\epsilon_{i}^{(j-1)}, \gamma_{j-1}\right\rangle+e_{j-1}\left\langle\epsilon_{i}^{(j)}, \lambda_{j}-\lambda_{j-1}\right\rangle=p_{i}^{(j)} \eta\left(\nu_{i}^{(j-1)}\right)+e_{j-1} q_{i}^{(j)} .
$$

We deal with the equality for $b_{i}^{(j)}$ in a similar way. We have that $\xi_{j}\left(\nu_{i}^{(j)}\right)=\sum_{l=1}^{d}\left\langle\epsilon_{i}^{(j)}, \varepsilon_{l}^{(0)}\right\rangle+$ $\sum_{l=1}^{j-1}\left(1-n_{l}\right)\left\langle\epsilon_{i}^{(j)}, \gamma_{l}\right\rangle+\left\langle\epsilon_{i}^{(j)}, \gamma_{j}\right\rangle$. By (44) and relations (3) we get:

$$
\xi_{j}\left(\nu_{i}^{(j)}\right)=p_{i}^{(j)} \xi_{j-1}\left(\nu_{i}^{(j-1)}\right)+\left\langle\epsilon_{i}^{(j)}, \gamma_{j}-n_{j-1} \gamma_{j-1}\right\rangle=p_{i}^{(j)} \xi_{j-1}\left(\nu_{i}^{(j-1)}\right)+q_{i}^{(j)} .
$$

In both cases the result follows by the induction hypothesis. 
Proposition 9.12. We have that $C P(f, w)=\cup_{j=1}^{g} \cup_{i=1}^{d}\left\{\left(\eta\left(\nu_{i}^{(j)}\right), \xi_{j}\left(\nu_{i}^{(j)}\right)\right\} \cup\{(1,1)\}\right.$.

Proof. The equality of these two lists is consequence of Lemma 9.11 We use also that $\epsilon_{d+g+1} \in \sigma_{g+1}$ verifies that $\eta\left(\epsilon_{d+g+1}\right)=1, \xi_{g+1}\left(\epsilon_{d+g+1}\right)=1$.

We discuss now some properties related with special vectors (see Definition 3.15).

Remark 9.13. By Definition 3.15 the vector $\nu_{i}^{(j)} \in \rho_{j}$ is special if the conditions $1 \leq i^{\prime} \leq d$, $q_{i^{\prime}}^{\left(j^{\prime}\right)}=0$ for $j^{\prime}=1, \ldots, j-1$ and $p_{i^{\prime}}^{\left(j^{\prime}\right)}=n_{j}, q_{i^{\prime}}^{\left(j^{\prime}\right)}=1$, hold if and only if $i=i^{\prime}$ and in addition we have that $q_{i}^{(j+1)}>0$ if $j<g$. If $\nu_{i}^{(j)}$ is a special vector then $\nu_{i}^{(l)}=\epsilon_{i}$ for $1 \leq l \leq j-1$. By Lemma 9.2 and Notation 2.19 we get that $\xi_{j}\left(\nu_{i}^{(j)}\right)=n_{j}+1$ and $\eta\left(\nu_{i}^{(j)}\right)=n_{j} \cdots n_{g}$. In this case we have that $\left(\xi_{j}\left(\nu_{i}^{(j)}\right), \eta\left(\nu_{i}^{(j)}\right)\right) \neq\left(\xi_{j}\left(\nu_{l}^{(j)}\right), \eta\left(\nu_{l}^{(j)}\right)\right)$ for $1 \leq l \leq d$ and $l \neq i$.

Definition 9.14. The local contribution $L C_{j}$ at level $j$ to the naive motivic zeta function is

$$
L C_{j}:=(\mathbf{L}-1)^{d+1}\left(S_{\sigma_{j}^{+}}+S_{\sigma_{j}^{-}}+S_{\sigma_{j+1}^{-}}\right)+(\mathbf{L}-1)^{d}(\mathbf{L}-2) S_{\rho_{j}},
$$

where if $j=g$ one must replace $\sigma_{g+1}^{-}$for $\sigma_{g+1}$.

The following result is analogous to [3, Proposition 5.4]:

Proposition 9.15. If $\nu_{i}^{(j)}$ is a special vector then there exists a polynomial $R_{j} \in \mathbf{Z}\left[\mathbf{L}^{ \pm 1}\right][T]$ such that

$$
L C_{j}=R_{j} \prod_{r=j-1}^{j+1} \prod_{l=1, l \neq i}^{d}\left(1-L^{-\xi_{r}\left(\nu_{l}^{(r)}\right)} T^{\eta\left(\nu_{l}^{(r)}\right)}\right)^{-1} \prod_{r=j-1, r \neq j}^{j+1}\left(1-L^{-\xi_{r}\left(\nu_{i}^{(r)}\right)} T^{\eta\left(\nu_{i}^{(r)}\right)}\right)^{-1} .
$$

Proof. Assume first that $j<g$. By the proof of Theorem 3.7 there exists a polynomial $R_{j}^{\prime} \in \mathbf{Z}\left[\mathbf{L}^{ \pm 1}\right]$ such that $L C_{j}=R_{j}^{\prime} \prod_{r=j-1}^{j+1} \prod_{l=1}^{d}\left(1-L^{-\xi_{r}\left(\nu_{l}^{(r)}\right)} T^{\eta\left(\nu_{l}^{(r)}\right)}\right)^{-1}$.

The vector $\bar{v}_{i}^{(j)}:=\frac{\left(n_{j}-1\right)}{n_{j}} \nu_{i}^{(j)}+\frac{1}{n_{j}} e_{d+j}\left(\right.$ resp. $\epsilon_{i}$ ) belongs to $\sigma_{j}^{+} \cap \mathbf{Z}^{d+g+1}$ (resp. $\sigma_{j}^{-} \cap \mathbf{Z}^{d+g+1}$ ). We denote by $\Theta^{\prime}$ the minimal subdivision of the fan $\Theta$ which contains the cones $\tau_{j}^{+}:=\rho_{j}+$ $\bar{v}_{i}^{(j)} \mathbf{R}_{\geq 0} \subset \sigma_{j}^{+}$and $\tau_{j}^{-}:=\rho_{j}+e_{i} \mathbf{R}_{\geq 0} \subset \sigma_{j}^{-}$.

Notice that $\xi_{j}\left(\bar{v}_{i}^{(j)}\right)=n_{j}$ and $\eta\left(\bar{v}_{i}^{(j)}\right)=\left(n_{j}-1\right) n_{j+1} \cdots n_{g}$ and also $\xi_{j}\left(\epsilon_{i}\right)=1$ and $\eta\left(\epsilon_{i}\right)=0$.

It is easy to see that $\bar{v}_{i}^{(j)}$ (resp. $\left.e_{i}\right)$ together with the elements of a basis of the lattice $\phi_{j}^{*}\left(N_{j}\right)$ are part of a basis of $\mathbf{Z}^{d+g+1}$ hence we obtain that

$$
S_{\tau_{j}^{+}}=S_{\rho_{j}} \mathbf{L}^{-\xi_{j}\left(\bar{v}_{i}^{(j)}\right)} T^{\eta\left(\bar{v}_{i}^{(j)}\right)}\left(1-\mathbf{L}^{-\xi_{j}\left(\bar{v}_{i}^{(j)}\right)} T^{\eta\left(\bar{v}_{i}^{(j)}\right)}\right)^{-1} \text { and } S_{\tau_{j}^{+}}=S_{\rho_{j}} \mathbf{L}^{-1}\left(1-\mathbf{L}^{-1}\right)^{-1} .
$$

We can decompose then the local contribution as a sum $L C_{j}=(\mathbf{L}-1)^{d+1}\left(S_{\sigma_{j+1}^{-}}+\sum_{\tau} S_{\tau}\right)+$ $(\mathbf{L}-1)^{d}(\mathbf{L}-2) S_{\rho_{j}}$, where $\tau \neq \rho_{j}$ runs through the cones of $\Theta^{\prime}$ which meet the interior of $\sigma_{j}^{-}$or of $\sigma_{j}^{+}$.

The linear map $\Upsilon_{j+1}: N_{j+1}^{\prime} \rightarrow \mathbf{Z}^{d+g+1}$ was defined in Lemma 6.13. One has that $\Upsilon_{j+1}(0,1)=$ $\epsilon_{d+1}+\sum_{i=j+2}^{g+1} n_{j+1} \cdots n_{i-1} \epsilon_{d+i}$. Hence $\xi_{j+1}\left(\Upsilon_{j+1}(0,1)\right)=1$ and $\eta\left(\Upsilon_{j+1}(0,1)\right)=n_{j+1} \cdots n_{g}$. By Lemma 6.13 we deduce that $S_{\Upsilon_{j+1}\left(\rho^{\prime}\right)}=S_{\rho_{j}} \frac{\mathbf{L}^{-1} T^{n_{j+1} \cdots n_{g}}}{1-\mathbf{L}^{-1} T^{n_{j+1} \cdots n_{g}}}$.

By these observations and the equality (25) it is enough to check that

$$
\begin{aligned}
L C_{j}^{\prime} & :=(\mathbf{L}-1)^{d+1}\left(S_{\Upsilon_{j+1}\left(\rho^{\prime}\right)}+S_{\tau_{j}^{+}}+S_{\tau_{j}^{-}}\right)+(\mathbf{L}-1)^{d}(\mathbf{L}-2) S_{\rho_{j}} \\
& =R_{j}^{\prime \prime} \frac{\mathbf{L}^{-n_{j}} T^{\left(n_{j}-1\right) n_{j+1} \cdots n_{g}}}{\left(1-\mathbf{L}^{-n_{j}} T^{\left(n_{j}-1\right) n_{j+1} \cdots n_{g}}\right)} \frac{\mathbf{L}^{-1} T^{n_{j+1} \cdots n_{g}}}{\left(1-\mathbf{L}^{-1} T^{n_{j+1} \cdots n_{g}}\right)} \prod_{l=1, l \neq i} \frac{L^{-\xi_{r}\left(\nu_{l}^{(r)}\right)} T^{\eta\left(\nu_{l}^{(r)}\right)}}{\left(1-L^{-\xi_{r}\left(\nu_{l}^{(r)}\right)} T^{\eta\left(\nu_{l}^{(r)}\right)}\right)},
\end{aligned}
$$

for some polynomial $R_{j}^{\prime \prime} \in \mathbf{Z}\left[\mathbf{L}^{ \pm 1}\right][T]$. The term $L C_{j}^{\prime}$ is equal to

$$
\begin{aligned}
& (\mathbf{L}-1)^{d}\left(S_{\rho_{j}}+(\mathbf{L}-1) S_{\rho_{j}} \frac{\mathbf{L}^{-n_{j}} T^{\left(n_{j}-1\right) n_{j+1} \cdots n_{g}}}{1-\mathbf{L}^{-n_{j}} T^{\left(n_{j}-1\right) n_{j+1} \cdots n_{g}}}+(\mathbf{L}-1) S_{\rho_{j}} \frac{\mathbf{L}^{-1} T^{n_{j} \cdots n_{g}}}{1-\mathbf{L}^{-1} T^{n_{j+1} \cdots n_{g}}}+(\mathbf{L}-2) S_{\rho_{j}}\right) \\
& =(\mathbf{L}-1)^{d+1} S_{\rho_{j}}\left(1+\frac{\mathbf{L}^{-n_{j}} T^{\left(n_{j}-1\right) n_{j+1} \cdots n_{g}}}{1-\mathbf{L}^{-n_{j}} T^{\left(n_{j}-1\right) n_{j+1} \cdots n_{g}}}+\frac{\mathbf{L}^{-1} T^{n_{j+1} \cdots n_{g}}}{1-\mathbf{L}^{-1} T^{n_{j+1} \cdots n_{g}}}\right) \\
& =(\mathbf{L}-1)^{d+1} S_{\rho_{j}}\left(\frac{1-\mathbf{L}^{-\left(n_{j}+1\right)} T^{n_{j} \cdots n_{g}}}{\left(1-\mathbf{L}^{-n_{j}} T^{\left(n_{j}-1\right) n_{j+1} \cdots n_{g}}\right)\left(1-\mathbf{L}^{-1} T^{n_{j+1} \cdots n_{g}}\right)}\right) \text {. }
\end{aligned}
$$


Finally, notice that the greatest common divisor of the terms $1-\mathbf{L}^{-\left(n_{j}+1\right)} T^{n_{j} \cdots n_{g}}$ and $1-$ $\mathbf{L}^{-n_{j}} T^{\left(n_{j}-1\right) n_{j+1} \cdots n_{g}}$ (resp. of $1-\mathbf{L}^{-\left(n_{j}+1\right)} T^{n_{j} \cdots n_{g}}$ and $1-\mathbf{L}^{-1} T^{n_{j+1} \cdots n_{g}}$ ) is equal to one.

The proof in the case $j=g$ follows by a similar argument.

Proof of Corollary 3.17. We use the formula for the naive motivic zeta function of Theorem 3.7. By definition if $1 \leq i \leq d$ there exists at most one integer $1 \leq j \leq g$ such that $\nu_{i}^{(j)}$ is special. If $1 \leq i, l \leq d, i \neq l$ and the vectors $\nu_{i}^{(j)}$ and $\nu_{l}^{(r)}$ are special for $1 \leq j, r \leq g$ then $j \neq r$. In this case then the pairs $\left(\xi_{j}\left(\nu_{i}^{(j)}\right), \eta\left(\nu_{i}^{(j)}\right)=\left(n_{j}+1, n_{j} \cdots n_{g}\right)\right.$ and $\left(\xi_{r}\left(\nu_{l}^{(r)}\right), \eta\left(\nu_{l}^{(r)}\right)=\left(n_{r}+\right.\right.$ $\left.1, n_{r} \cdots n_{g}\right)$ are linearly independent, hence the greatest common divisor of $1-\mathbf{L}^{-n_{j}-1} T^{n_{j} \cdots n_{g}}$ and $1-\mathbf{L}^{-n_{l}-1} T^{n_{l} \cdots n_{g}}$ is equal to one. These conditions guarantee that we can apply Proposition 9.15 any time we have special vectors.

Then the proof for the topological zeta function follows by applying Proposition 1.11.

Remark 9.16. By the results obtained in this section it is easy to see that the set of strongly candidate poles for the motivic zeta function introduced in [3] is equal to

$$
S C P(f, w):=\cup_{j=1}^{g} \cup_{i=1}^{d}\left\{\left(\eta\left(\nu_{i}^{(j)}\right), \xi_{j}\left(\nu_{i}^{(j)}\right) \mid \nu_{i}^{(j)} \text { non-special }\right\} \cup\{(1,1)\} .\right.
$$

The set $\operatorname{SCP}(f, w)$ of candidate poles coincides with the one we get from Corollary 3.17. This implies that the arguments in the proof of the monodromy conjecture in 3] Chapter 6, are not affected by the inaccuracy of the formulas for the zeta functions in 3 . (see Remarks 3.10 and 3.18 .

\section{ExAmple}

Example 10.1. The q.o. polynomial $f=\left(z^{2}-x y^{3}\right)^{4}-x^{4} y^{13}$ is and has characteristic exponents $\lambda_{1}=(1 / 2,3 / 2)$ and $\lambda_{2}=(1 / 2,7 / 4)$. The generators of the semigroup are $\gamma_{1}=(1 / 2,3 / 2)$ and $\gamma_{2}=(1,13 / 4)$. We have that $n_{1}=2$ and $n_{2}=4, \ell_{1}=\ell_{2}=2$ and $r_{1}=r_{2}=1$. The conic integral complex $\Theta$ associated to $f$ is represented by the Figure 3. We have that

$$
\left.c_{1}\left(\sigma_{1}^{+}\right)=c_{1}\left(\sigma_{2}^{-}\right)=\left[\mu_{4}\right], \quad c_{1}\left(\sigma_{1}^{-}\right)\right)\left[\mu_{8}\right], \quad c_{1}\left(\sigma_{2}^{+}\right)=c_{1}\left(\sigma_{3}\right)=1
$$

and also $c_{1}\left(\rho_{1}\right)=\left[\left\{(x, y) \in\left(\mathbf{C}^{*}\right)^{2} \mid\left(y^{2}-x\right)^{4}=1\right\}, c_{1}\left(\rho_{2}\right)=\left[\left\{(x, y) \in\left(\mathbf{C}^{*}\right)^{2} \mid y^{4}-x=1\right\}\right.\right.$. The multiplicities of the simplicial cones are $\operatorname{mult}\left(\rho_{1}\right)=\operatorname{mult}\left(\sigma_{2}^{-}\right)=2, \operatorname{mult}\left(\sigma_{1}^{+}\right)=\operatorname{mult}\left(\sigma_{2}^{+}\right)=4$, and $\operatorname{mult}\left(\rho_{2}\right)=\operatorname{mult}\left(\sigma_{3}\right)=1$.

The motivic Milnor fibre is

$$
S_{f, 0}=c_{1}\left(\rho_{1}\right)(1-\mathbf{L})+\left[\mu_{8}\right] \mathbf{L}+c_{1}\left(\rho_{2}\right)(1-\mathbf{L})-\left[\mu_{4}\right] \mathbf{L}(1-\mathbf{L})+(1-\mathbf{L})^{2} .
$$

The Hodge-Steenbrink spectrum is $h s p(f, 0)=\frac{1-t}{1-t^{1 / 8}} t-t=t^{\frac{9}{8}}+\cdots+t^{\frac{15}{8}}$. According to Theorem 3.7 the topological zeta function is

$$
Z_{\text {top }}(f, s)=\frac{24 s+13}{(3+8 s)(5+24 s)}+\frac{22+96 s}{(5+24 s)(3+8 s)(11+52 s)}-\frac{s}{(1+s)(3+8 s)(11+52 s)} .
$$

We compute this example with the method of [3] with respect to the form $\omega=d x \wedge d y \wedge d z$. The Newton map followed by a suitable translation $\mathcal{N}_{1}$ is given by $x=x_{1}^{2}, y=y_{1}^{2}, z=x_{1} y_{1}^{3}\left(z_{1}-1\right)$. We get $f \circ \mathcal{N}_{1}=x_{1}^{8} y_{1}^{2} 4\left(z_{1}^{4}-y_{1}^{2}+\cdots\right)$ and $\omega \circ \mathcal{N}_{1}=x_{1}^{2} y_{1}^{4} d x_{1} \wedge d y_{1} \wedge d z_{1}$.

Theorems 2.7 and 5.3 of [3] imply that $Z_{\mathrm{top}}(f, s)=\frac{24 s+13}{(3+8 s)(5+24 s)}+Z_{B}(s)$, where $\frac{24 s+13}{(3+8 s)(5+24 s)}$ is called the Part A in [3, Definition 2.3]. The term $Z_{B}(s)$ is the sum of three contributions corresponding to the three faces of the compact edge of the Newton polyhedron of $f \circ \mathcal{N}_{1}$. The contribution of the two zero-dimensional faces is equal to $J_{\sigma_{2}^{+}}(f, s)+J_{\sigma_{2}^{-}}(f, s)=\frac{22+96 s}{(5+24 s)(3+8 s)(11+52 s)}$. The contribution of this edge equals $\frac{-2 s}{(1+s)(3+8 s)(11+52 s)}$ because it has integral lenght two.

The disagreement with our formula appears since the Newton map $\mathcal{N}_{1}$ is not birational and the strict transform of $f$ by the Newton map is not irreducible (see Remark 9.9). 


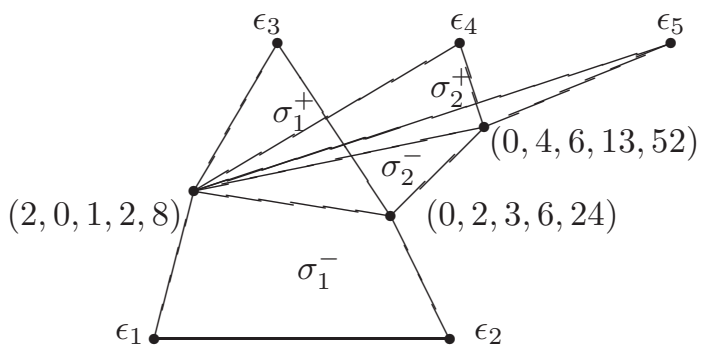

Figure 3. Projectivization of the fan $\Theta \subset \mathbf{Z}_{\geq 0}^{5}$ of $f=\left(z^{2}-x y^{3}\right)^{4}+x^{4} y^{13}$.

\section{REFERENCES}

1. S. S. Abhyankar, On the ramification of algebraic functions, Amer. J. Math. 77 (1955), 575-592.

2. E. Artal Bartolo, Pi. Cassou-Noguès, I. Luengo, and A. Melle Hernández, The Denef-Loeser zeta function is not a topological invariant, J. London Math. Soc. (2) 65 (2002), no. 1, 45-54.

3. _ Quasi-ordinary power series and their zeta functions, Mem. Amer. Math. Soc. 178 (2005), no. 841.

4. _ On the log-canonical threshold for germs of plane curves, Singularities I, Contemp. Math., vol. 474, Amer. Math. Soc., Providence, RI, 2008, pp. 1-14.

5. J. Denef and F. Loeser, Caractéristiques d'Euler-Poincaré, fonctions zêta locales et modifications analytiques, J. Amer. Math. Soc. 5 (1992), no. 4, 705-720.

6. __ Motivic Igusa zeta functions, J. Algebraic Geom. 7 (1998), no. 3, 505-537.

7. - Germs of arcs on singular algebraic varieties and motivic integration, Invent. Math. 135 (1999), no. 1, 201-232.

8. _ Geometry on arc spaces of algebraic varieties, European Congress of Mathematics, Vol. I (Barcelona, 2000), Progr. Math., vol. 201, Birkhäuser, Basel, 2001, pp. 327-348.

9. Lefschetz numbers of iterates of the monodromy and truncated arcs, Topology 41 (2002), no. 5, 1031-1040.

10. __ Motivic integration, quotient singularities and the McKay correspondence, Compositio Math. 131 (2002), no. 3, 267-290.

11. P. Du Bois and F. Michel, The integral Seifert form does not determine the topology of plane curve germs, J. Algebraic Geom. 3 (1994), no. 1, 1-38.

12. A. H. Durfee, Fibered knots and algebraic singularities, Topology 13 (1974), 47-59.

13. G. Ewald, Combinatorial convexity and algebraic geometry, Graduate Texts in Mathematics, vol. 168, Springer-Verlag, New York, 1996.

14. W. Fulton, Introduction to toric varieties, Annals of Mathematics Studies, vol. 131, Princeton University Press, Princeton, NJ, 1993, The William H. Roever Lectures in Geometry.

15. Y.-N. Gau, Embedded topological classification of quasi-ordinary singularities, Mem. Amer. Math. Soc. 74 (1988), no. 388, 109-129, With an appendix by Joseph Lipman.

16. P. D. González Pérez, Singularités quasi-ordinaires toriques et polyèdre de Newton du discriminant, Canad. J. Math. 52 (2000), no. 2, 348-368.

17. ble) 53 (2003), no. 6, 1819-1881.

18. _ The semigroup of a quasi-ordinary hypersurface, J. Inst. Math. Jussieu 2 (2003), no. 3, 383-399.

19. M. González Villa, Función zeta motívica de singularidades quasiordinarias irreducibles, Tesis Doctoral, Universidad Complutense de Madrid, 2010.

20. G. Guibert, Espaces d'arcs et invariants d'Alexander, Comment. Math. Helv. 77 (2002), no. 4, 783-820.

21. G. Guibert, F. Loeser, and M. Merle, Iterated vanishing cycles, convolution, and a motivic analogue of a conjecture of Steenbrink, Duke Math. J. 132 (2006), no. 3, 409-457.

22. H.W.E. Jung, Darstellung der funktionen eines algebraischen körpers zweier unabhängiger veränderlichen $x, y$ in der umgebung einer stelle $x=a, y=b$, J. Reine Angew. Math. 133 (1908), 289-314.

23. G. Kempf, F. F. Knudsen, D. Mumford, and B. Saint-Donat, Toroidal embeddings. I, Lecture Notes in Mathematics, Vol. 339, Springer-Verlag, Berlin, 1973.

24. V. S. Kulikov, Mixed Hodge structures and singularities, Cambridge Tracts in Mathematics, vol. 132, Cambridge University Press, Cambridge, 1998.

25. A. Landman, On the Picard-Lefschetz transformation for algebraic manifolds acquiring general singularities, Trans. Amer. Math. Soc. 181 (1973), 89-126.

26. J. Lipman, Topological invariants of quasi-ordinary singularities, Mem. Amer. Math. Soc. 74 (1988), no. 388, $1-107$.

27. — Equisingularity and simultaneous resolution of singularities, Resolution of singularities (Obergurgl, 1997), Progr. Math., vol. 181, Birkhäuser, Basel, 2000, pp. 485-505.

28. L. J. McEwan and A. Némethi, The zeta function of a quasi-ordinary singularity, Compos. Math. 140 (2004), no. 3, 667-682. 
29. J. Milnor, Singular points of complex hypersurfaces, Annals of Mathematics Studies, No. 61, Princeton University Press, Princeton, N.J., 1968.

30. V. Navarro Aznar, Sur la théorie de Hodge-Deligne, Invent. Math. 90 (1987), no. 1, 11-76.

31. T. Oda, Convex bodies and algebraic geometry, Ergebnisse der Mathematik und ihrer Grenzgebiete (3) [Results in Mathematics and Related Areas (3)], vol. 15, Springer-Verlag, Berlin, 1988

32. C. A. M. Peters and J. H. M. Steenbrink, Mixed Hodge structures, Ergebnisse der Mathematik und ihrer Grenzgebiete. 3. Folge. Vol. 52, Springer-Verlag, Berlin, 2008.

33. P. Popescu-Pampu, On the analytical invariance of the semigroups of a quasi-ordinary hypersurface singularity, Duke Math. J. 124 (2004), no. 1, 67-104.

34. M. Saito, Mixed Hodge modules, Publ. Res. Inst. Math. Sci. 26 (1990), no. 2, 221-333.

35. _ On Steenbrink's conjecture, Math. Ann. 289 (1991), no. 4, 703-716.

36. Exponents of an irreducible plane curve singularity, arXiv:math/0009133 2 (2000).

37. K. Sakamoto, The Seifert matrices of Milnor fiberings defined by holomorphic functions, J. Math. Soc. Japan 26 (1974), 714-721.

38. R. Schrauwen, J. H. M. Steenbrink, and J. Stevens, Spectral pairs and the topology of curve singularities, Complex geometry and Lie theory (Sundance, UT, 1989), Proc. Sympos. Pure Math., vol. 53, Amer. Math. Soc., Providence, RI, 1991, pp. 305-328.

39. R. P. Stanley, Enumerative combinatorics. Vol. 1, Cambridge Studies in Advanced Mathematics, vol. 49, Cambridge University Press, Cambridge, 1997.

40. J. H. M. Steenbrink, Mixed Hodge structure on the vanishing cohomology, Real and complex singularities, Sijthoff and Noordhoff, Alphen aan den Rijn, 1977, pp. 525-563.

41. ㄴ. The spectrum of hypersurface singularities, Astérisque (1989), no. 179-180, 11, 163-184.

42. A. N. Varchenko, Asymptotic Hodge structure on vanishing cohomology, Izv. Akad. Nauk SSSR Ser. Mat. 45 (1981), no. 3, 540-591, 688.

iCMat. Fac. de CC. Matemáticas. Univ. Complutense de Madrid. Plaza de las Ciencias 3. 28040. MADRID. SPAIN.

E-mail address: pgonzalez@mat.ucm.es

Fac. de CC. Matemáticas. Univ. Complutense de Madrid. Plaza de las Ciencias 3. 28040. Madrid. Spain and Match, Univ. Heidelberg, Im Neuenheimer Feld 288, 69120 Heidelberg, Germany

E-mail address: mgv@mat.ucm.es, villa@mathi.uni-heidelberg.de 